\title{
Fabrication and Characterization of Hybrid Photosensitive Protein/Conducting Polymer Devices for Optical Sensing
}

\author{
Harel Lichtenstein, B.Eng.
}

\begin{abstract}
A thesis submitted to the
Faculty of Graduate and Postdoctoral Affairs

in partial fulfillment of the requirements for the degree of
\end{abstract}

Master of Applied Science in Electrical Engineering

Ottawa-Carleton Institute for Electrical and Computer Engineering

Department of Electronics and Computer Engineering

Carleton University

Ottawa, Ontario

December, 2016

(C) Copyright

Harel Lichtenstein, 2016 


\begin{abstract}
The DC, light intensity and frequency response of a bR/PEDOT:PSS interfaced organic optoelectronic lateral device was studied. The processing steps required for the formation of such device, as well as alternative processing methods for PEDOT:PSS were presented as well. A measurable output of the device when irradiated with light of different wavelengths was obtained, as well as relationships between light intensity and photoresponse of the device via resistance measurements. Finally, the indirect measurement of resistance change due to modulation frequency of the incident light was obtained.
\end{abstract}




\section{Acknowledgments}

I would like to thank:

My supervisor, Professor Steve McGarry, for all of his insight and guidance along this project.

Rob Vandusen and Angela McCormick with all of their help, time and effort.

Alasdair Rankin, Scott Ferguson and Svetlana Demtchenko for their guidance.

To all the artists that supplied me with music for the hours of work.

And to all my Friends and Family. 


\section{Table of Contents}

Abstract $\quad$ iii

Acknowledgments $\quad$ iv

Table of Contents $\quad$ v

List of Tables $\quad$ vii

List of Figures $\quad$ viii

Nomenclature $\quad$ x

1 Introduction $\quad 1$

1.1 Motivation . . . . . . . . . . . . . . . . . . 1

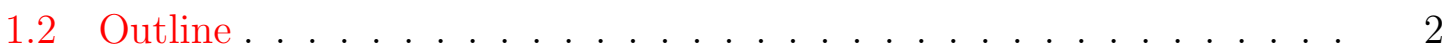

2 Background $\quad 3$

2.1 Bacteriorhodopsin . . . . . . . . . . . . . . . 3

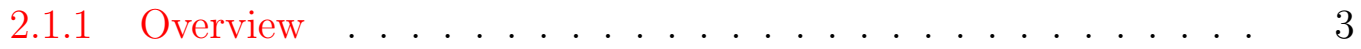

2.1.2 Ion Pumping Mechanism . . . . . . . . . . . . . . . . . 3

2.1.3 Absorption Spectrum . . . . . . . . . . . . 5

2.2 Conducting Polymers . . . . . . . . . . . . . . . . 7

2.2.1 Chemical Structure . . . . . . . . . . . . . . 8

2.2.2 PEDOT:PSS Charge Transfer . . . . . . . . . . . 15

2.3 Application . . . . . . . . . . . . . . . . 20

3 Literature Review $\quad 21$

3.1 bR Potential Applications . . . . . . . . . . . . . . . . 21

3.2 PEDOT:PSS Potential Applications . . . . . . . . . . . . 26 
3.3 PEDOT:PSS/bR Device Potential Applications . . . . . . . . . . . 30

4 Design And Fabrication $\quad 35$

4.1 Introduction . . . . . . . . . . . . . . . . . . . . 35

4.1 Device Design . . . . . . . . . . . . . . . . 35

4.1 .2 Mask Design . . . . . . . . . . . . . . 36

4.2 PEDOT:PSS Spin Coating . . . . . . . . . . . . . 37

4.3 Reactive Ion Etching . . . . . . . . . . . . . . . . . . 41

4.3.1 Gas Etch Rate . . . . . . . . . . . . . . . . . 41

4.4 Lift-off . . . . . . . . . . . . . . . . . . . . . 42

4.5 Metal Deposition . . . . . . . . . . . . . . . . 45

4.6 bR Deposition . . . . . . . . . . . . . . . . . . 47

5 Results and Discussion $\quad 49$

5.1 Mobility Measurements . . . . . . . . . . . . . . . . 50

5.2 DC Photoresponse Measurements . . . . . . . . . . . . . . . . 54

5.3 Frequency Response . . . . . . . . . . . . . . . . . 63

$\begin{array}{lll}6 & \text { Conclusion } & 70\end{array}$

6.1 Summary of Knowledge Contribution . . . . . . . . . . . . . . . . 70

6.2 Improvements and Future Work . . . . . . . . . . . . . . . 71

$\begin{array}{ll}\text { List of References } & 73\end{array}$ 


\section{List of Tables}

4.1 Number of devices . . . . . . . . . . . . . . . . . . 37

4.2 Size of Pads . . . . . . . . . . . . . . . . . . . . . 38

4.3 Table of Ellipsometry Results . . . . . . . . . . . . . . . . . . . . . . 40

4.4 PEDOT:PSS Spin Casting processing . . . . . . . . . . . . . . 43

4.5 PEDOT:PSS Lift-off process (without OmniCoat) . . . . . . . . 46

5.1 Tested Devices . . . . . . . . . . . . . . . . 49

5.2 Thickness and Resistance of New vs Old PEDOT:PSS spin device . . 51

5.3 Resistivity and Conductivity of a PEDOT:PSS film . . . . . . . . 52

5.4 Tested Devices . . . . . . . . . . . . . . 55

5.5 Tested Devices . . . . . . . . . . . . . . . . 60

5.6 Fixed resistors used for voltage divider . . . . . . . . . . . . 65 


\section{List of Figures}

2.1 Tertiary structure of $\mathrm{bR} \ldots \ldots \ldots \ldots \ldots \ldots$

2.2 bR Photocycle. . . . . . . . . . . . . . . . . 5

2.3 Conformal change of all-trans-retinal, the main mechanism of the proton pumping in $\mathrm{bR} \ldots \ldots \ldots \ldots \ldots \ldots$

2.4 Schematic of the conformational changes a bR protein will undergo to pump an ion $[6] \ldots \ldots \ldots \ldots \ldots \ldots \ldots \ldots$

2.5 Spectral absorption peak changes in bR and its intermediate states [3] 8

2.6 Constructive and Destructive atomic orbital interference $\ldots \ldots$. . . 9

2.7 Antibonding and Bonding Molecular Orbitals . . . . . . . . . 10

2.8 (a) $\sigma$ bonding between two $p_{z}$ orbitals (b) $\pi$ bonding between two $p_{x}$

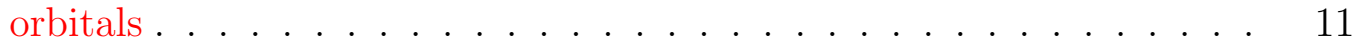

2.9 Chemical structure of Polyethelyne _ . . . . . . . . . . . 12

2.10 Hybridization of a $s$ with $3 p$ orbitals into four $2 s p^{3}$ orbitals $\ldots \ldots 13$

2.11 Chemical structure of Polyacetelyne . . . . . . . . . . . . . 13

2.12 (a) $\sigma$ bonding between two $p_{z}$ orbitals (b) $\pi$ bonding between two $p_{x}$ orbitals . . . . . . . . . . . . . . . . . . . . 14

2.13 1-D chain of repeating ion sequences, each dot can be considered a monomer . . . . . . . . . . . . . . . . . . 15

2.14 Band formation for through orbital hybridization (With permission from $[11]) \ldots \ldots \ldots \ldots \ldots \ldots$

2.15 Chemical structure of PEDOT:PSS (With permission from [11]) . . 17

2.16 (a) Chemical Structure of PEDOT:PSS. (b) Individual Oligomers of PEDOT on a long PSS chain. (c) Entangled PEDOT:PSS Chains. (d) Particles of entangled PEDOT:PSS chains. (With permission from [11]) 18

3.1 bR based photosensitive pixel $[17] \ldots \ldots \ldots \ldots \ldots \ldots$

3.2 Proposed circuit model of bR $[17] \ldots \ldots \ldots \ldots \ldots$

3.3 Spectral absorption of wildtype and mutants of bR [18] . . . . . 24 
3.4 Schematic of bR Photocycle memory cycle, with $\mathrm{P} / \mathrm{O}$ states as the "On" bit and bR state as the "Off" bit . . . . . . . . . . . . . 25

3.5 Parallel write (left) and read (right) sequence of 3 bits of data using the branched cycle architecture propsed in Figure $3.4[20]$. . . . . . . 26

3.6 EL Device with PEDOT:PSS as a transparent electrode . . . . . . . . 28

3.7 OLED Stack with a PEDOT:PSS/AU NPs HIL . . . . . . . . . . 29

3.8 Flexible touchscreen pixel $[31] \ldots \ldots$. . . . . . . . . . . . . 33

4.1 High level architecture of the bR/PEDOT:PSS device . . . . . . . . 36

4.2 Design of (a) device defining mask and (b) contact defining mask . . 38

4.3 Areas of Ellipsometry Measurement . . . . . . . . . . . . . . . 40

4.4 Wafer with mask ready for etching . . . . . . . . . . . . . . . . . . 42

4.5 Emulsion mask for lithographic etching of PR . . . . . . . . . . . . 45

4.6 Wafer with mask ready for metallization . . . . . . . . . . . . 47

4.7 bR/PEDOT:PSS complete device . . . . . . . . . . . . 48

$4.8 \mathrm{bR} / \mathrm{PEDOT}$ :PSS complete device . . . . . . . . . . . . 48

5.1 Current dependant Voltage of two PEDOT:PSS devices . . . . . . . . 51

5.2 Number of Photons irradiated by a 532nm Green Laser in a $1.5 \times 1.5 \mathrm{~mm}$

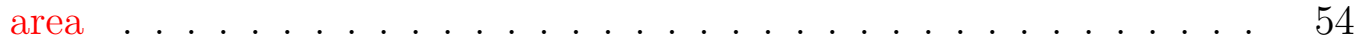

5.3 Circuit schematic model proposed for a PEDOT:PSS resistive strip where the Green area represents the illuminated bR/PEDOT:PSS in-

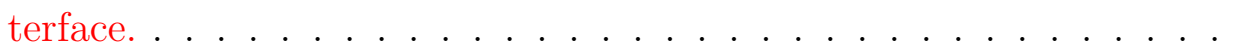

5.4 Dimensions for all the resistor sections in a bR/PEDOT:PSS device of size A1. . . . . . . . . . . . . . . . . . . . .

5.5 PEDOT:PSS film resistance dependant on incident $532 \mathrm{~nm}$ Laser Power for A and B sized devices . . . . . . . . . . . . . . . . 61

5.6 PEDOT:PSS film resistance dependant on incident $532 \mathrm{~nm}$ Laser Power for C and D sized devices . . . . . . . . . . . . . . 62

5.7 Testing setup for the measurement of bR/PEDOT:PSS frequency re-

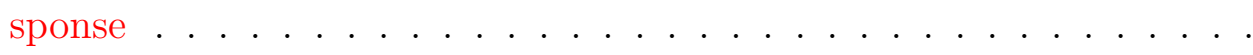

5.8 Frequency dependant response of bR/PEDOT:PSS devices of size A

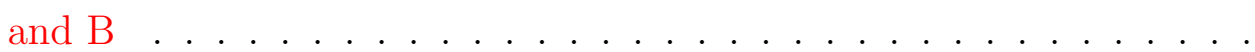

5.9 Frequency dependant response of bR/PEDOT:PSS device devices of size $\mathrm{C}$ and D . . . . . . . . . . . . . . . . 


\section{Nomenclature}

\begin{tabular}{||cc||}
\hline Symbol & Meaning \\
\hline $\mathrm{bR}$ & Bacteriorhodopsin \\
\hline PEDOT:PSS & poly(3,4-ethylenedioxythiophene) polystyrene sulfonate \\
\hline$\lambda$ & Wavelength \\
\hline$N_{T}(t)$ & Total Number of molecules in bR \\
\hline$N_{B}(t)$ & Number of bR molecules in bR state \\
\hline$N_{M}(t)$ & Number of bR molecules in M state \\
\hline $\mathrm{q}$ & Elementary charge \\
\hline$\sigma$ & Conductivity \\
\hline$\rho$ & Resistivity \\
\hline$\mu_{p}$ & Bulk Hole Mobility \\
\hline $\mathrm{p}$ & Number of holes in semiconductor device \\
\hline$E_{\lambda}$ & Energy of photon of wavelength $\lambda$ \\
\hline $\mathrm{h}$ & Planck's Constant \\
\hline $\mathrm{c}$ & Speed of Light \\
\hline$N_{p}$ & Number of Photons \\
\hline
\end{tabular}




\section{Chapter 1}

\section{Introduction}

\subsection{Motivation}

Organic material such as conducting polymers and proteins have been studied for potential used in biosensors for a long time. These materials, once integrated with modern Silicon technology could provide a wide range of sensing applications as well as cost reduction. New capabilities may also be possible for computation and signal processing. In terms of computational power, today's CMOS based IC offer unparalleled performance and speed. However, computers have always had a hard time performing tasks of cognition and pattern recognition. This is where realizing a fully organic neural network could add a new dimension to computing. Neural network "cells" operate on changing resistance through conducting paths which can represent neural synapses and Conducting polymers could potentially fulfil this role due to their good electrical properties, integration, availability and low cost.

To fully realize an organic neural network which would mimic a human brain, it would also need a visual input to be able to start the "thinking" process and generate an output. For this, the protein Bacteriorhodopsin (bR) which has an photoresponse analogue to the visual rhodopsins in human eyes is being studied as a light transducer. When combining the photoexcitation response of bR with a conducting polymer, a fully organic visual input implementation can be realized, as the protein can trigger a change in the conductivity of the polymer which can then be propagated into the polymer based neural network. By using the transient voltage response of the bR photosensitive layer, the resistance of a conducting polymer can be modulated by light, effectively creating a photosensitive variable resistor.

The objective of this thesis is to explore the processing and testing of such an 
organic optoelectronic device which could potentially be implemented as the visual input, "the eyes", of a neural network.

\subsection{Outline}

Chapter 2 lays the theoretical foundation for the optoelectric device. It outlines the

photo cycle of Bacteriorhodopsin and explains the fundamentals of its ion pumping mechanism, as well as talking about conducting polymers chemical structure and conduction models. It then focuses specifically on PEDOT:PSS, the main conducting polymer used in this thesis.

Chapter 3 gives a brief overview of the current applications of both bR and PEDOT:PSS, and the work that has been presented in interfacing these two materials. This will help give some scope to the thesis.

Chapter 4 describes in detail all the processing steps followed to create the device for this thesis, as well as exploring other possible alternate processing steps for conducting polymers which may be useful in the future integration with more conventional CMOS processes.

Chapter 5 will provide a summary of measurements of the device, including response characterization, as well as a detailed look at topographical processing results.

Finally, chapter 6 will present the conclusion to this thesis, including opinions about the organic device implementation and potential future research. 


\section{Chapter 2}

\section{Background}

\subsection{Bacteriorhodopsin}

\subsubsection{Overview}

Bacteriorhodopsin $(\mathrm{bR})$ is a large protein $(26.8 \mathrm{kDa})$ found in the cell membrane of the Halobacteria halobium's Purple Membrane (PM). In bacteria, this protein acts as a protein pump which creates an electrochemical gradient across the cell membrane which is subsequently converted into chemical energy. The bR hexagonal crystalline lattice is composed of three identical protein chains (248 amino-acids residues), each composed of seven transmembrane alpha helices (named A to G) which contain a retinal chromophore embedded unto the 216th lysine residue [1]. A bR molecule's tertiary structure is show in Figure 2.1.

\subsubsection{Ion Pumping Mechanism}

A large amount of research hours have been put into deciphering the exact mechanism which makes bR such an effective ion pump (On average, 1 light quanta transports from 0.8 to 1.2 protons) [1].

The ion pumping mechanism is activated by the incidence of visible light unto the extracellular (EC) side, where the absorption of a light quanta is converted into an ion which is then released into the cytoplasmic side $(\mathrm{CP})$. Upon photo-excitation, bR will undergo a series of conformal changes (described in terms of photo-cycles, see Figure 2.2), all of which result in the change of the embedded retinal chromophore 


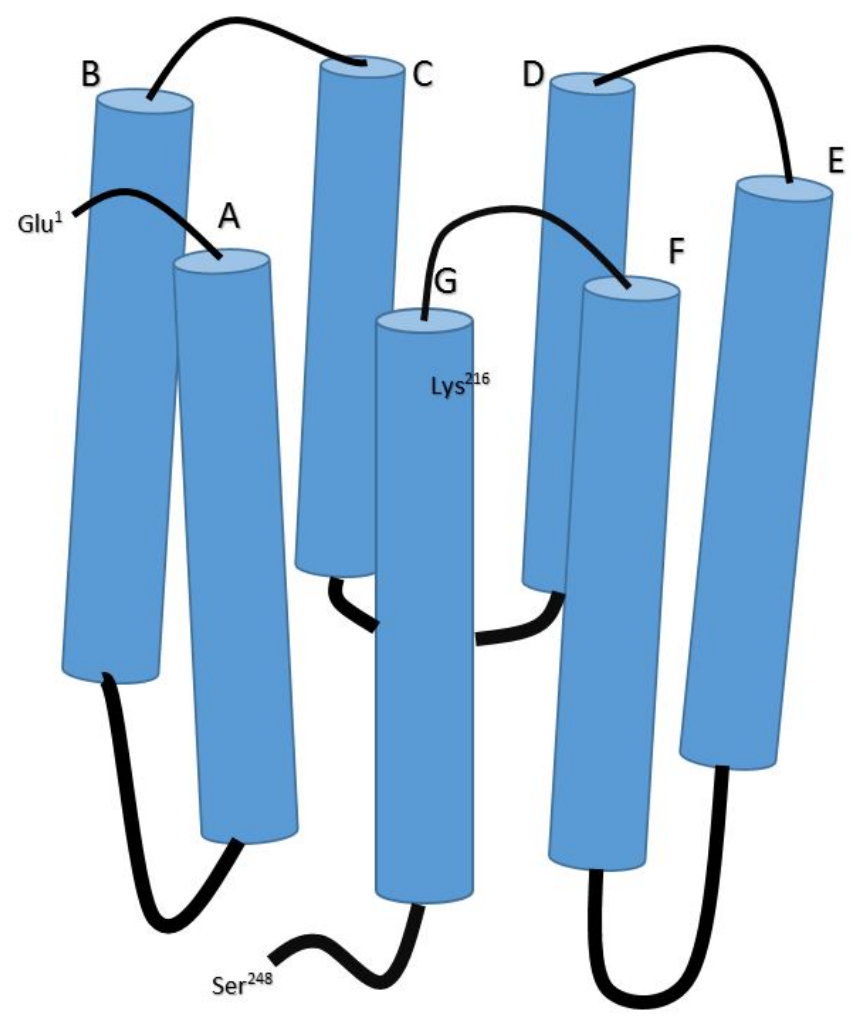

Figure 2.1: Tertiary structure of bR

from an all-trans to and all-cis molecular configuration (Figure 2.3).

Whilst on the ground state (knows as bR state), the retinal will remain in its all-trans configuration. Once a photon is absorbed the retinal will undergo several conformational changes into its new 13-cis isomer (M State, $\sim 3.5 \mathrm{pSec}$ ), and the excited Schiff base NH Group is now in able to donate a proton to an Aspertate molecule (Asp 85) embedded in the polyemr chain which is closer to the EC side [2]. Figure 2.3 will show a graphical representation of the cycle described below

This proton donation is aided by conformal change of the protein helices, bringing polypeptides closer to the Schiff Base in the embedded retinal. In around $1 \mu \mathrm{Sec}$ the Asp 85 accepts the proton (L State) and the $\mathrm{C}$ alpha helix will return to its original shape. The now deprotonated 13-cis-retinal will straighten and bend the helix $\mathrm{F}$ its embedded into, causing the Schiff base to get closer towards the CP side, where another Aspertate molecule (Asp 96) will donate its proton to the Schiff base (State M). This exchange takes about $50 \mu \mathrm{Sec}$. At the same time the conformational changes 


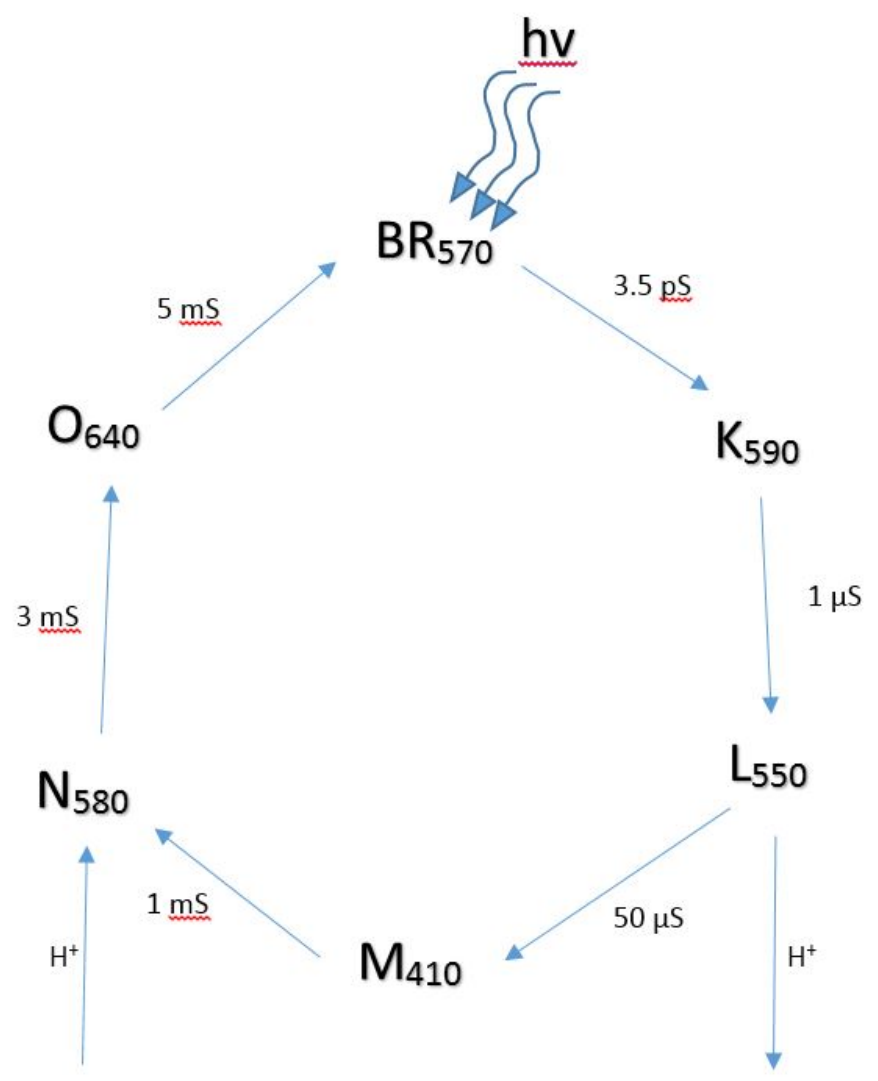

Figure 2.2: bR Photocycle

are occurring, the protonated Asp 85 will pump out its proton through a series of hydrogen bonds and eventually off the EC side. Almost simultaneously (about 1 $\mathrm{mSec}$ ) a proton will enter through the $\mathrm{CP}$ side and reprotonate the Asp 96, which has donated its own proton to the Schiff base. With the Schiff base being reprotonated, after $\sim 3 \mathrm{mS}$ the $\mathrm{F}$ helix will return to its original shape, however at this point the retinal is still in its 13 -cis configuration (O State), but after a short time $(\sim 5 \mathrm{mSec})$ it will decay back into its all-trans configuration bringing the protein back to its $\mathrm{bR}$ state. Figure 2.2 shows this cycle with timings and Figure 2.4 shows the change in absorption peaks

\subsubsection{Absorption Spectrum}

One of the most attractive features of bR lies with the photocycle described above, as every intermediate state shifts the maximum peak of absorption spectra within the 


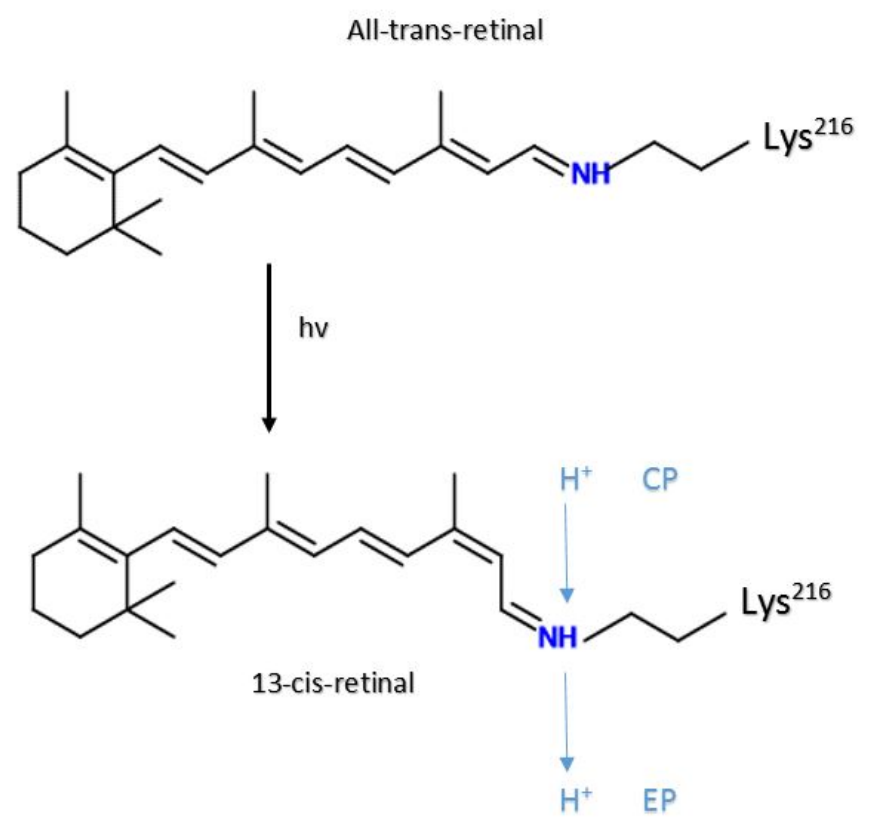

Figure 2.3: Conformal change of all-trans-retinal, the main mechanism of the proton pumping in $\mathrm{bR}$

visible range. [4]

Figure 2.4 shows the spectral change across all the intermediates of bR, and of most interest for various optical applications is the spectral change of the $\mathrm{M}$ state into the $412 \mathrm{~nm}$ blue light [3]. From the previous description of the photocycle, it can be seen that the bR molecule will thermally relax after $\sim 10 \mathrm{mSec}$ back into its bR state, however if blue light (close to the absorption spectrum) is irradiated on the sample, these thermal relaxation intermediates can be skipped, and the molecule can be brought back into its bR state, effectively acting as a optical switch [5]. It can be easily seen that this property could allow for various optical and opto-electrical applications (more on application in the next chapter).

There has been a lot of work done in creating bR mutants which will change the cycle of different intermediates. Some factors that have been used to modify bR include genetic modification, $\mathrm{pH}$ changes, drying time and temperature [6], but for the scope of this paper, the naturally found form (wild-type) will be used. 


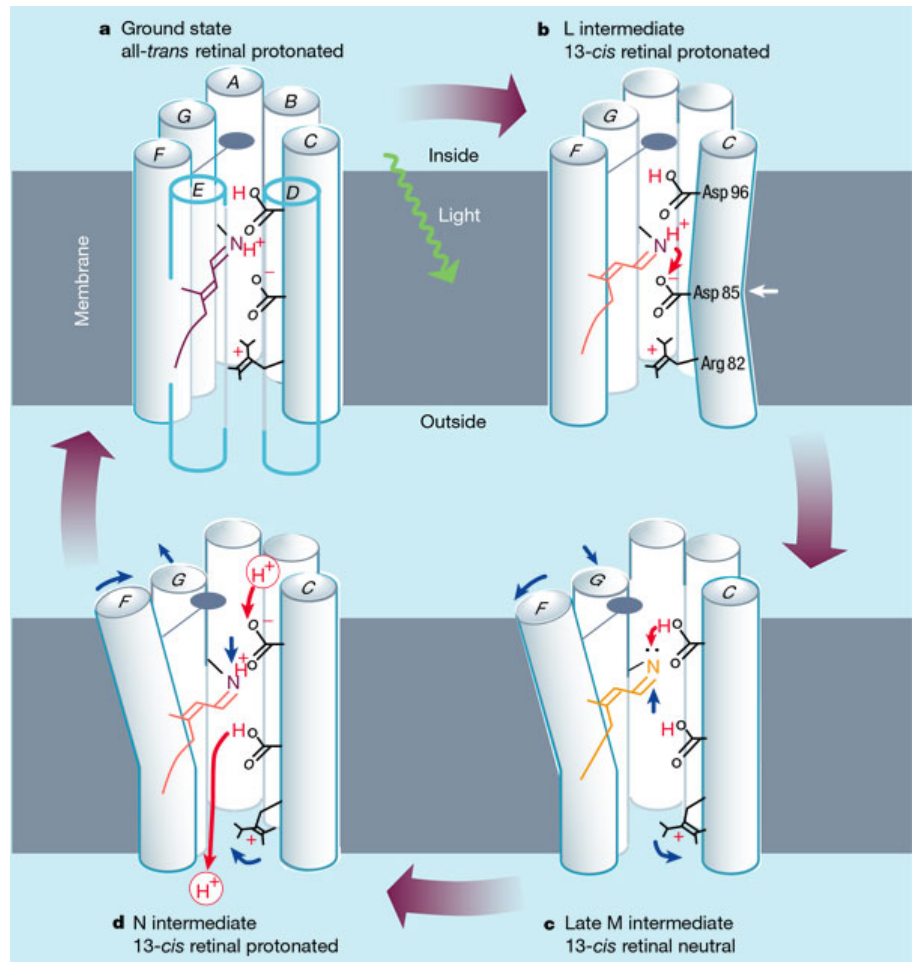

Figure 2.4: Schematic of the conformational changes a bR protein will undergo to pump an ion [6]

\subsection{Conducting Polymers}

Most of modern electronics are based on inorganic solids, since their conductivity can behave as a metal, insulator, or semiconductor. Polymers in the other hand, have always been thought of as strictly an insulator. However this notion changed around the 1950's where polymers with semiconductor properties were discovered, and it was further reinforced in the late $70 \mathrm{~s}$ when conductivities rivalling that of metals were achieved.

While both polymers and inorganic materials share similar conductive properties, their actual method of charge transport is quite different, as the latter has a rigid crystal lattice which depends on free hole/electron charge movement, while the former is a much more deformable structure, and as such depends on various quasi-particles due to charge-lattice coupling formed in the long polymer chains [7]. 


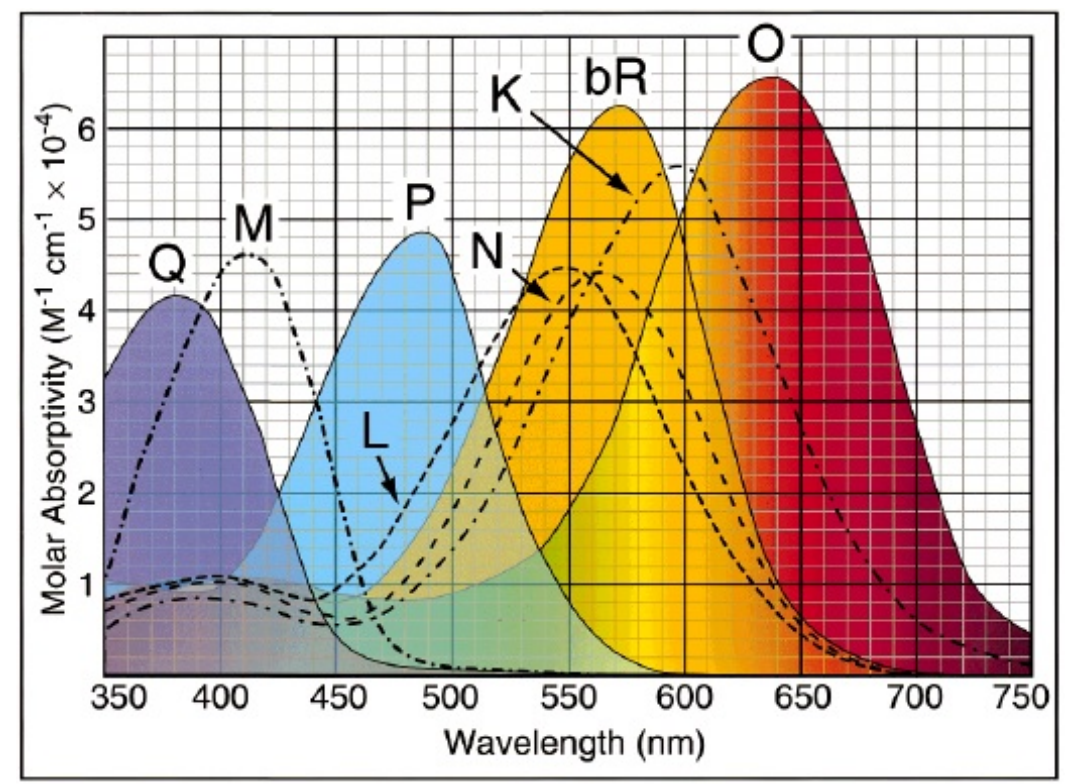

Figure 2.5: Spectral absorption peak changes in bR and its intermediate states [3]

\subsubsection{Chemical Structure}

\section{Molecular Bonding}

Polymers are a family of molecules which are made up of many repeated subunits linked by covalent bonds, which form into long chains. These organic polymers are based off of a backbone made up of carbon atoms which are covalently bonded to each other (ie. they share electrons), and may be bonded to other organic molecules like hydrogen, oxygen or sulfur to name a few [7].

To understand how covalent bonds help link polymer chains and grant them conducting properties, its important to understand how atomic orbitals are created when atoms come together to form a molecule. Atomic orbitals are defined as the probability of finding any electron in a specific space around the atom's nucleus (represented mathematically as the wave function), with the simplest example being a Hydrogen atom which only posses one electron and one proton. For this atom, the orbital exists in its $1 \mathrm{~s}$ form, as the wave function in the ground state displays a spherical symmetry, ie. the electron can statistically be found in a sphere around the atom [8].

The aforementioned orbital number, 1s, comes from the unique characterization of these atomic orbitals: a unique set of values based on the principal $(n)$, azimuthal $(l)$ and magnetic $\left(m_{l}\right)$. The principal quantum number corresponds to the electron's energy level. The azimuthal determines quantized orbital angular momentum of this 
electron within the orbital, and there can be 0 to $n$ - 1 values for the angular momentum. So relating these to the previously mentioned hydrogen atom, a 1s orbital would represent to a principal quantum number of 1 , with an angular momentum of $\mathrm{s}$, which represents 0 (long ago Bohr proposed the annotation $\mathrm{s}=0, \mathrm{p}=1, \mathrm{~d}=2, \mathrm{f}=3 \ldots$ ). The magnetic momentum along any axis can range from $-l$ to $l$. Finally, the last quantum number to describe the state of an electron in an atomic orbital is the quantized spin of an electron $\left(m_{s}\right)$ which describes the intrinsic angular momentum of the electron and can have a value of $-1 / 2$ or $1 / 2[8]$

\section{Bonding/Antibonding Orbitals}

The next step for is to understand what happens when two atoms come together to form a molecule. Continuing the example with hydrogen, once two molecules come together, the wavefunction of their single electron will overlap with each other, resulting in two molecular orbitals coming together and creating a bonding and an antibonding orbital, each caused by constructive and destructive interference, respectively (Figure 2.5).

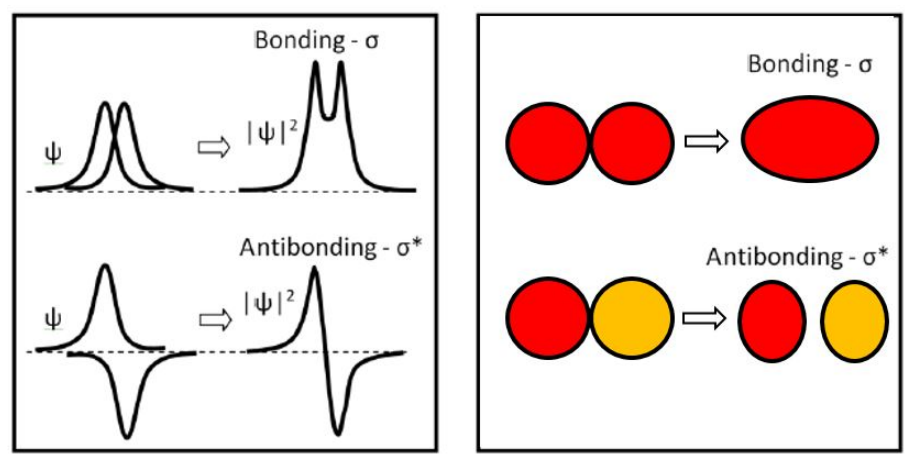

Figure 2.6: Constructive and Destructive atomic orbital interference

When talking about bonding orbitals, if the wavefunctions constructively interfere, it means that probability density of an electron being between the two nuclei is increased, which in turn decreases the electrostatic repulsion between the protons in two nuclei. This is beneficial to the Hydrogen molecule, as this would lower its overall energy level in comparison to a single Hydrogen atom. In contrast, when electrons occupy an antibonding orbital, the wavefunction creates a destructive interference. This means that the probability density of electrons being between the nuclei decreases, and increases the probability density of them being outside increases which 
in turn causes electrostatic repulsion between the protons in the nuclei, and causes the antibonding orbital to have an overall higher energy then the bonding orbital (Figure 2.6) [8].

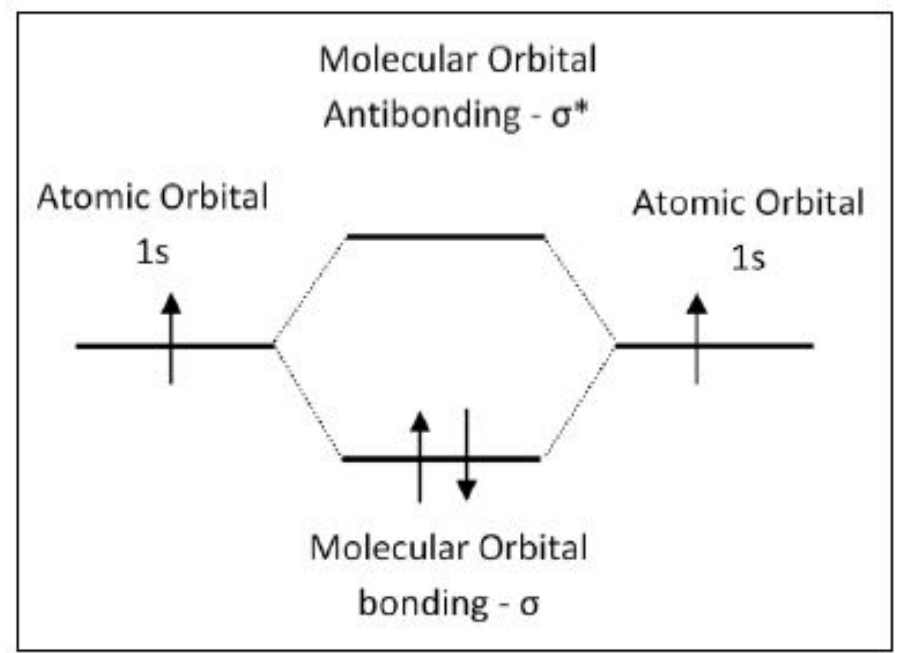

Figure 2.7: Antibonding and Bonding Molecular Orbitals

\section{Sigma and Pi Bonding}

Another property of molecular orbitals to consider is the plane in which the bondings lie with respect to each other. When the bonding and antibonding orbitals do not have a nodal plane along the internuclear axis (aka the axis along the nuclei) then this can be considered a Sigma $(\sigma)$ bond, where $\sigma$ and $\sigma^{*}$ denote the bonding and antibonding orbitals respectively (asterisk denotes antibonding). It quickly becomes obvious as to why this is the strongest type of molecular bond, since there is a much larger overlap in atomic orbitals. Conversely, if the probability of finding an electron (aka there is a nodal plane) over the internuclear axis, the molecules will form a Pi $(\pi)$ bond, in which the atoms are not free to rotate along the bond [8]. Figure 2.7 shows how two p orbitals can form a $\sigma$ or $\pi$ bond.

Since S orbitals display spherical symmetry, they have no orientation. However, p orbitals will display 3 orientations $\left(p_{x}, p_{y}, p_{z}\right)$, all of which are related to the aforementioned magnetic quantum number $m_{l}$. If an axis, say the $\mathrm{z}$ axis, is arbitrarily assigned as the internuclear axis, then the overlap between two $p_{z}$ oribitals will result in a $\sigma_{p z}$ and $\sigma *_{p z}$ molecular bonds. With such geometry defined, the overlap between 
(a)

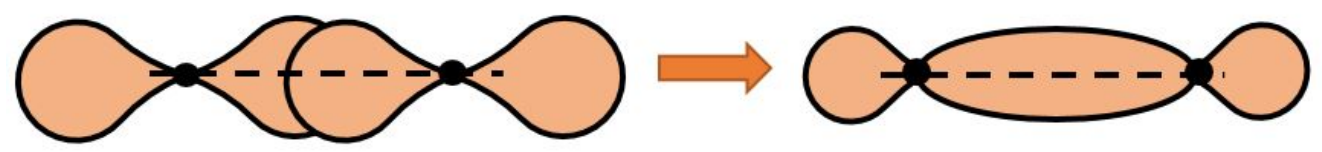

(b)

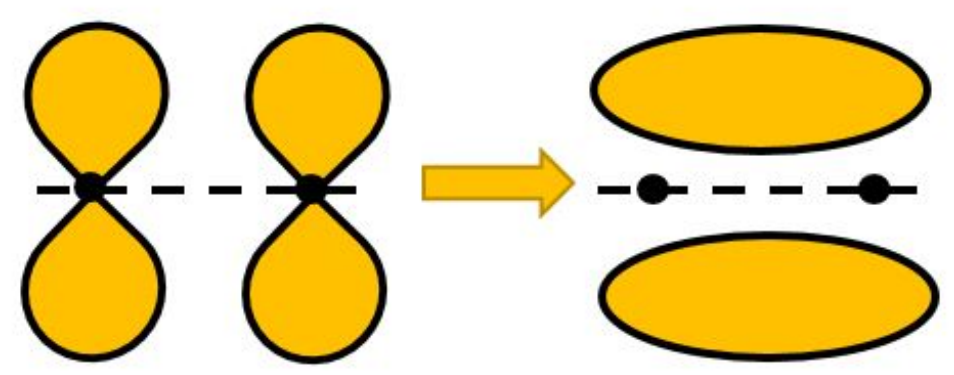

Figure 2.8: (a) $\sigma$ bonding between two $p_{z}$ orbitals (b) $\pi$ bonding between two $p_{x}$ orbitals

two $p_{x}$ (and therefore two $p_{y}$ ) orbitals would result in molecular orbitals that have nodes along the internucluear axis, and therefore there would be $\pi_{x}$ and $\pi *_{x}$ orbitals. Even though every molecular bond between two atoms will have bonding and antibonding, for the scope of this thesis, all bonds will be called either $\sigma$ or $\pi$ without specifically referencing the antibonding [8].

\section{Hybrid Orbitals in Saturated and Unsaturated Polymers}

As mentioned in the previous section, atoms try to arrange their bonds as to lower the total energy of the molecule. Since conducting polymers have a backbone of linear carbon chains, they will tend to form two types of hybrid structures to accomplish a lower energy state [9]. These structures are called tetrahedral and hexagonal geometries, with Tetrahedral referring to nonconducting materials such as Nylon or other saturated polymers, while hexagonal geometry refers to conducting materials such as PEDOT:PSS and conjugated (unsaturated) polymers. To further understand these hybrid structures, an overview of atomic orbital hybridization is needed.

Sometimes, the $p$ and $s$ orbitals of atoms will mix and create hybrid orbitals, which overlap with orbitals from other atoms to form molecular orbitals. This process is 
referred to as hybridization of atomic orbitals. The basic concept of hybridization is through electron promotion into a higher state orbital, the atomic orbital can actually achieve a lower more favourable energy state [9].

For the example of a Tetrahedral structure, a really simple hydrocarbon like Polyethylene (PE) can be studied (shown in Figure 2.8 below).

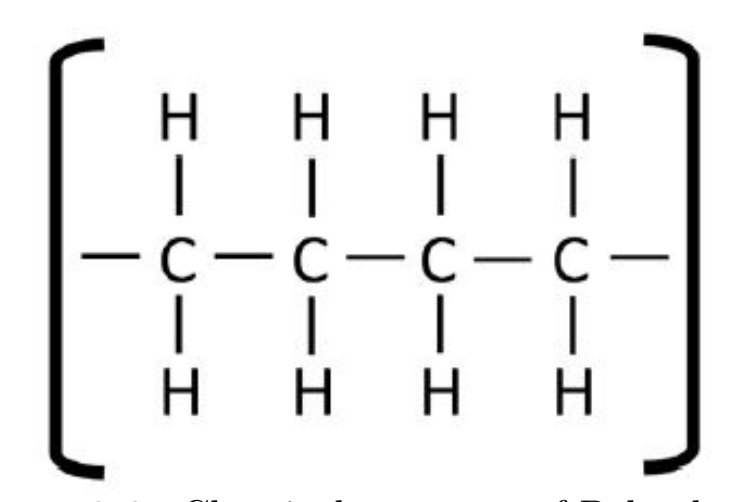

Figure 2.9: Chemical structure of Polyethelyne

PE has 4 valance electrons of a carbon atom, which are found in its $2 s$ and $2 p$ orbitals, but to become energy favourable the electrons will be rearranged into 4 hybrid orbitals, each holding a single unpaired electron. This is done by promoting an electron from the $2 s$ to the $2 p_{z}$ orbital, leaving 4 orbital with an unpaired electron. With this new arrangement (1 electron in the $2 s$ and 3 in the $2 p$ ), the four orbitals will mix into a linear combination to form four new hybrid orbitals called $2 s p^{3}$ orbitals (Figure 2.9). These hybrid orbitals have the same shape and energy (principal quantum number is 2), but will now have different orientations and form a regular tetrahedron structure.

With this new arrangement, two hydrogen atoms can now bond to two $2 s p^{3}$ orbitals and form a covalent $\sigma$ molecular bond, while the remaining hybrid orbitals can form $\sigma$ covalent bonds with two neighbouring carbon atoms, creating the polymers chain. These polymer chains with a backbone made out of carbon atoms with $\sigma$ bonds made from the $s p^{3}$ hybrid orbitals are called saturated polymers. With all the electrons in the hybrid orbitals being occupied with $\sigma$ covalent bonds, there are no electrons left for conduction and as such, saturated polymers are insulators [7].

For the case of a conducting polymer, there would also be a $2 s$ electron promoted to the $2 p_{z}$ orbital (like in the previous structure), however this time only $2 p$ orbitals and $1 s$ orbital will undergo hybridization into a $2 s p^{2}$ orbital, such that the carbon 


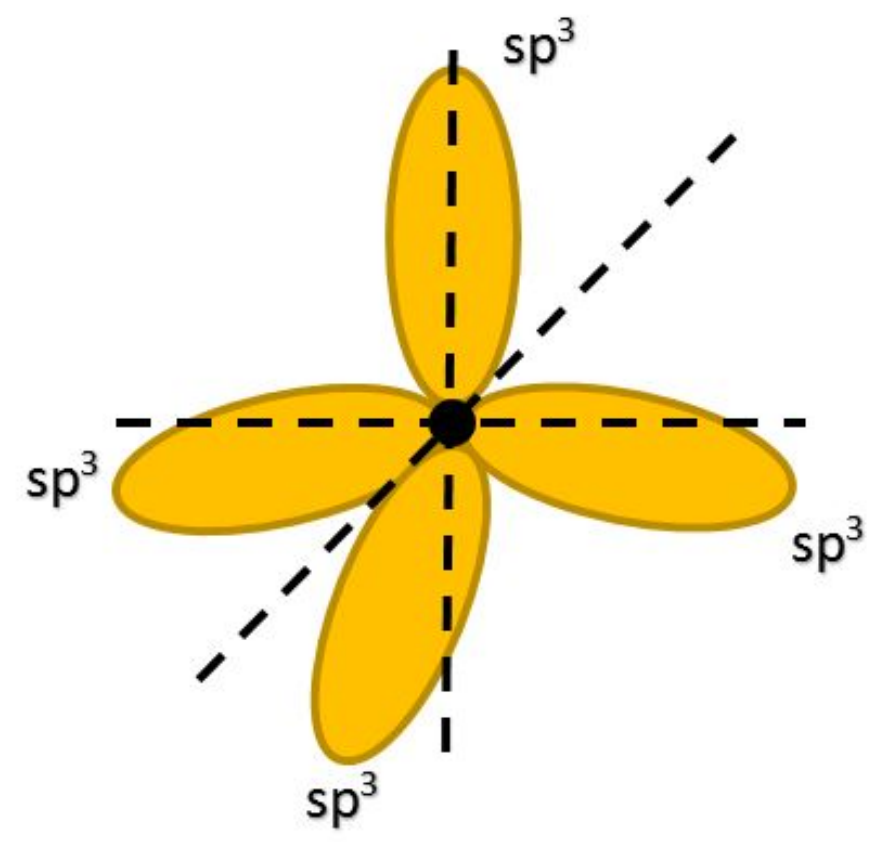

Figure 2.10: Hybridization of a $s$ with $3 p$ orbitals into four $2 s p^{3}$ orbitals

atom now has three $2 s p^{2}$ and one $2 p$ orbitals. Like in the tetrahedral case, the carbon will bind to its neighbouring carbon atoms by overlap of one of their $s p^{2}$ orbitals to create a $\sigma$ molecular bonds. The remaining $s p^{2}$ will form a $\sigma$ molecular bond with a hydrogen atom. The remaining unpaired electron the $2 p$ orbital can now form a $\pi$ bond with the unpaired electron of the adjacent carbon atom. This $\pi$ bond will be parallel to the $\sigma$ bond, effectively forming a double bond between the carbons. Polymers with this type of $s p^{2}$ hybridization are called unsaturated, while carbon chains with this type of $\pi$ bonding are called conjugated $\pi$ systems [7]. Polyacetylene, one of the more common conjugated $\pi$ systems can be seen in Figure 2.10.

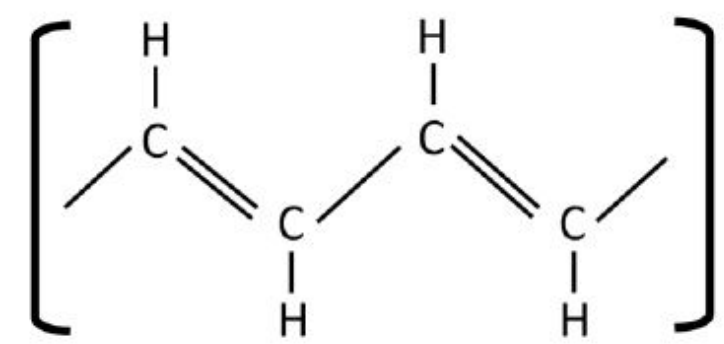

Figure 2.11: Chemical structure of Polyacetelyne

Finally, the other type of bond that can occur is a triple bond, made up of one $\sigma$ 

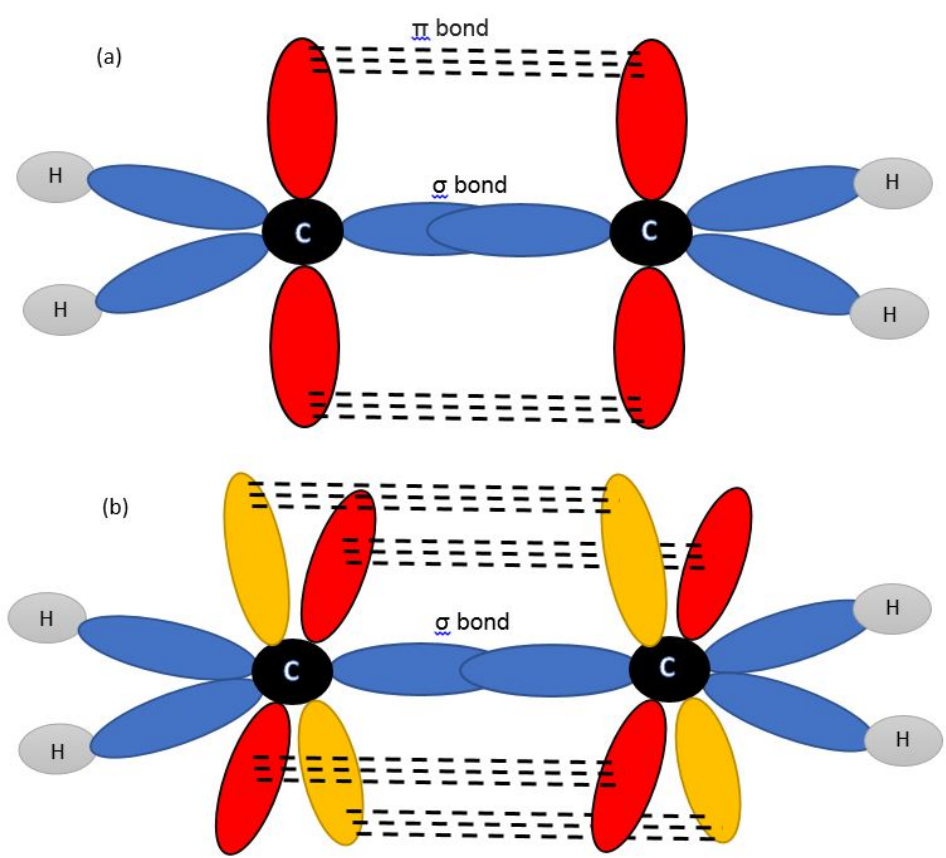

Figure 2.12: (a) $\sigma$ bonding between two $p_{z}$ orbitals (b) $\pi$ bonding between two $p_{x}$ orbitals

bonds and two $\pi$ bond. Here, the hybridization occurs between the $2 s$ orbital and one $2 p$ orbital, creating a $2 s p$ hybrid orbital which leaves two $2 p$ orbitals. These orbitals have an unpaired electron each, and can therefore form a $\sigma$-bond between the 2sp of each carbon atom, and the remaining $2 s p$ orbital in each atom can bond with a hydrogen. Also, each $2 p$ orbital from one carbon atom can form a $\pi$ bond with the adjacent carbon atom. In this case however, there are two $2 p$ orbitals in each carbon atom, therefore there can be two $\pi$ bonds, making it so that there are two $\pi$ bonds and a $\sigma$ bond, causing the molecule to have a triple bond (Figure 2.11)

Something to note is that unsaturated polymers are less chemically stable than saturated polymers, due to most of the electrons being found in the $\sigma$ bonds, where they are harder to remove compared to electrons found in the $\pi$ bonds. But as mentioned before, unsaturated polymers can exhibit semiconducting and sometimes even metallic properties, unlike saturated polymers [7]. 


\subsubsection{PEDOT:PSS Charge Transfer}

\section{Energy Band Structure of Conjugated Polymers}

In the last section covering molecular orbitals, only two atoms were considered when forming the molecule. Here, the molecular orbitals of these atoms are degenerate in energy. However as the two atoms come together, the degenerate atomic orbitals split into two non-degenerate (different energy) molecular orbitals. In polymers, as more molecules aggregate into a long chain consisting of $\mathbf{N}$ ion cores evenly separated by a distance a (Figure 2.12), atomic orbitals will split into a nondegenrate energy levels. But as the $N$ gets much larger, the spacing between these energy levels gets smaller and smaller, to the point they become a continuous energy band [10].

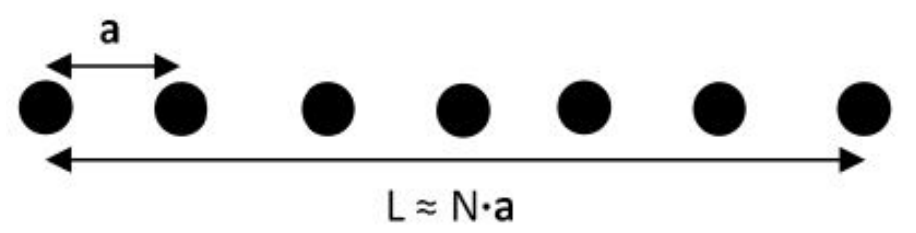

Figure 2.13: 1-D chain of repeating ion sequences, each dot can be considered a monomer

These energy bands can now either overlap or be separated by an energy gap known as the bandgap, with each energy band having a specific amount of occupying electrons based on temperature under no external field. This property is what defines materials' conduction: metals have partially filled energy bands that allow electrons to move to unoccupied levels under an electric field. Insulating materials will have a bandgap of several $\mathrm{eV}$ separating the filled (valence) from the unfilled (conduction) bands, making it such that thermal excitation of an electric field cannot make the electrons become free charge carriers. Finally, a semiconductor will have an energy gap small enough that electrons in the valence band can be excited into a free conduction state in the conduction band through thermal excitation or an electric field [10]. Figure 2.13 shows this principle in action, with the discrete atomic $2 \mathrm{p}$ and $2 \mathrm{~s}$ orbitals on the right being hybridized to $s p^{3}$ on the left hand side, forming the valance and conduction bands.

As mentioned in the previous chapter, conjugated polymers attribute their conduction to the delocalized $\pi$-electron structures they posses. In terms of molecular orbitals, this can be considered as the Highest Occupied Molecular Orbital (HOMO). 


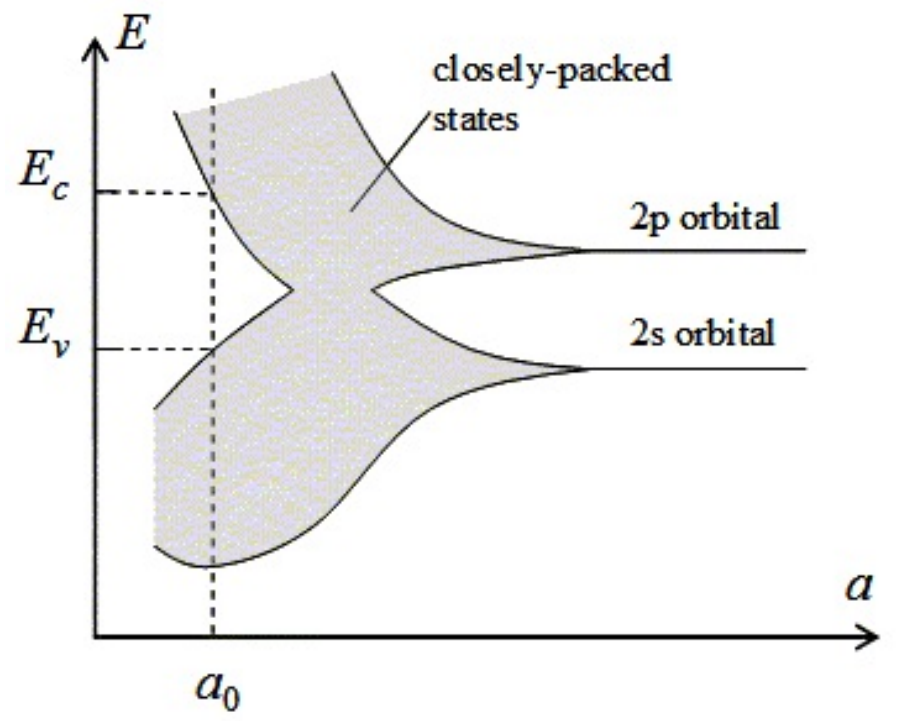

Figure 2.14: Band formation for through orbital hybridization (With permission from [11])

Corresponding to the delocalized $\pi$-electron, there is also an unoccupied $\pi^{*}$ orbital ready to accept electrons, known as the Lowest Unoccupied Molecular Orbital (LUMO). If we consider a long polymer chain, much like the combination of atom, the $\pi$ and $\pi^{*}$ band form a valance and conduction band respectively as the polymer chain repeats, so in terms of conjugated polymers the filled valance band can be considered the HOMO band, and the unfilled conduction band can be considered the LUMO band.

\section{PEDOT:PSS Structure}

Although the majority of this thesis has been based around the PEDOT:PSS complex, it wasn't until somewhat recently that the combination of these came to be. Before this, in 1980,(poly(ethylene-3,4-dioxythiophene))(PEDOT) was developed in the Bayer AG Labs. While it showed very promising properties for a conducting polymer, such as high conductivity and films being almost transparent in the oxidized state; the lack of solubility of the polymer with conventional solvents was a serious drawback for the application of printed electronics. However, the introduction of poly(styrenesulfonic acid) (PSS) creates the PEDOT:PSS complex (Figure 2.14) which allows for the formation of a dispersion of the polymer nanoparticles in water which allows for a much easier processing of a conductive film using various techniques, such as ink-jet, screen printing, spin coating and vacuum deposition [12]. A 
varied range of applications for this complex exist, such as antistatic coatings, electroluminescent devices, hole injecting layers in OLEDs, organic transistors and pixel displays to name a few.

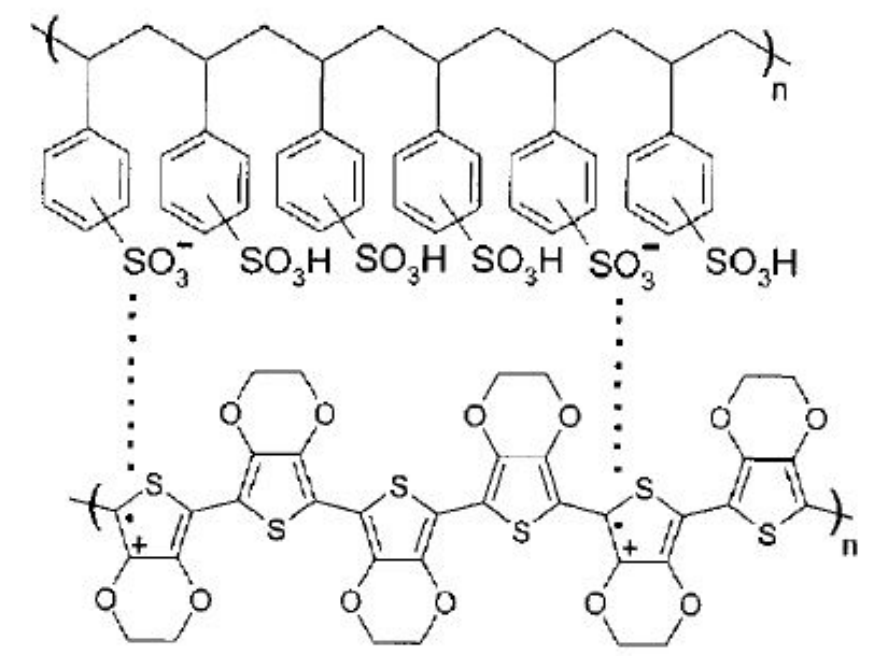

Figure 2.15: Chemical structure of PEDOT:PSS (With permission from [11])

Another positive effect of the addition of PSS is its use as a charge-compensation counter-polyanion for the positively charged PEDOT. PEDOT is referred as a polycation since each EDOT monomer can lose a $\pi$ electron, becoming positively charged. The PSS is considered a polyanion, since the $\mathrm{SO}_{3} \mathrm{H}$ group can lose its hydrogen (proton) and become negative. An ionic bond can now be created between the PSS polyanion and EDOT polycation, effectively p-doping the EDOT monomer. With this, the EDOT olingomers will grow into a PEDOT chain along a long PSS Polymer chain which acts as a backbone for the these shorter PEDOT chains, which have some of their thiophene group (sulfur aromatic ring) oxidized causing them to attach to the PSS backbone. It should be noted that in the PEDOT chains, there will be a hole for each ionic bond created, which in turns increases conductivity of the film. Conductivities are increased in mixtures where the ratio of PEDOT to PSS (by weight) is closer to $1: 1$ [12].

Various studies such as Gangopadhyay et al [13] have allowed for accurate modeling of the primary structure of PEDOT:PSS, on a molecular level (Figure 2.14). Kirchenmeyer et al [12] proposed a secondary structure, which consists of charged oligimers (6-18 repeating units) of EDOT distributed along a PSS chain backbone, with the EDOT binding to the sulfonic group anions (Figure 2.15(b)). A tertiary 
structure was also proposed (Figure 2.15(c)), consisting of entangled particles made from the PEDOT:PSS chains, which once aggregated into a mixture consisting of approximately 90 to $95 \%$ water, allow for the formation of a printable dispersion (Figure $2.15(\mathrm{~d}))$.

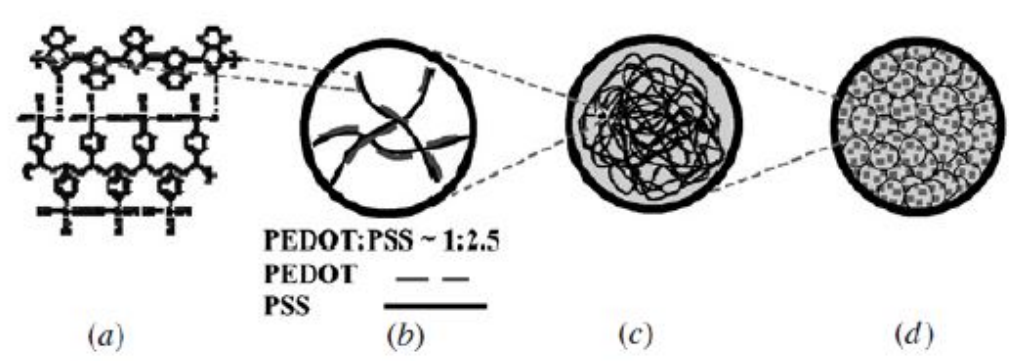

Figure 2.16: (a) Chemical Structure of PEDOT:PSS. (b) Individual Oligomers of PEDOT on a long PSS chain. (c) Entangled PEDOT:PSS Chains. (d) Particles of entangled PEDOT:PSS chains. (With permission from [11])

An important factor for PEDOT:PSS film conductivity is its morphology, as PEDOT:PSS films are known to have an anisotropic morphology. Various studies on the morphology and topology of PEDOT:PSS films have been done, for example Timpanaro et al. (2004) [14], who performed a study on these films using STM and AFM, showing that these films have a lamellar structure, with PEDOT clusters embedded in a PSS matrix. These studies show that the surface morphology and bulk of the film exhibit very different PEDOT cluster sizes, using AFM on cleaved samples to show that PEDOT:PSS films have PEDOT-rich "islands", typically of 20-30 $\mathrm{nm}$ diameter (in-plane) and height (out-of-plane) of 4-6 $\mathrm{nm}$. However, other granule sizes have been demonstrated to change the conductivity [12]. Separating these islands, PSS lamellas exist in the out of plane direction with not much separation in the in plane direction. The tertiary structure of the films consists of entangled complexes of these PEDOT rich islands and PSS lamellas, which tend to coil up in multiple directions. It has been shown that further manipulation of the coils to create more directionality could potentially improve the conductivity of these films.

\section{PEDOT:PSS Charge transfer}

From the studies mentioned in the previous section, a PEDOT:PSS film can be thought of as a mixture of PEDOT-rich conductive regions and non-conductive PSSrich regions where there is no PEDOT present. The conduction of current therefore, 
is composed of transfer of charge carriers from one PEDOT-rich island to another and as such, the ratio of PEDOT to PSS is a significant contributor to the overall conductivity of the film. For example, Clevios PH1000 (Conductivity $\sim 1000 \mathrm{~S} / \mathrm{cm}^{2}$ ) has a ratio of PEDOT:PSS (w/w) of 1:2.5, while Clevios Al4083 (0.002-0.0002 S/ $\left.\mathrm{cm}^{2}\right)$ has a ratio of 1:6. By this same idea, addition of foreign non-conductive materials can both decrease or increase the conductivity, with dymethil sulfoxide being a very common surfactant added to both improve the wetability and conductivity of PEDOT:PSS films. C.S. Pathak et al [15] did a study in which it decreased the resistivity of PEDOT:PSS films by almost two orders of magnitude. The mechanics of conduction increase are not fully understood, but Raman spectroscopy suggest that DMSO treatment causes a rearrangements of the PEDOT islands and washes away the PSS insulating areas from the surface, creating a better pathway for charge transport in the film. This should highlight the very important fact that morphology of PEDOT:PSS films plays a tremendously large role in its overall conductivity, leading to lots of research in the field of film confirmation to increase conductivity.

Herein also lies a major problem with PEDOT:PSS (and conjugated polymers in general), as the great variability in conductivity and its dependence in morphology has led to a lack of understanding of an appropriate and well understood model for conductivity, despite a wide range of research and applications in which PEDOT:PSS is currently being used in. The disorder of the polymer chains does not allow for a simple model of conductivity like that of other crystalline semiconductor materials. Models applied to these crystalline devices defines an electron experiencing little scattering due to the symmetry of the crystal lattice, has a well defined momentum and is delocalised. As disorder is introduced into the lattice, the electron will encounter more scattering, resulting in a conductivity and mean free path decrease. If enough disorder is introduced, the mean free path length becomes comparable to the lattice constant, causing the electron to lack a well defined moment, and to be spatially localised. Now, the electron can still move, but only by thermally activated hopping from one localised state to the other.

This has been brought up as a conduction model for disordered systems such as conducting polymers, in which the inherit disorder of the polymer chains dominate the conduction and as such, the localised charge hopping called Variable Range Hoping (VRH), normally used in amorphous inorganic semiconductors, seems to be taken as one of the more appropriate models for conduction, especially when talking about 
charge transport in PEDOT:PSS.

A study by Nardes et al. (2007) [16] studied how the anisotropic morphology of PEDOT:PSS films affected the in-plane and out-of-plane conductivities of spincoated films. The study concluded that conduction mechanisms in the in-plane and out-of-plane directions were due to 3 dimensional VHR and nearest-neighbour hopping, respectively. Another important discovery in the conduction model brought up by this study was the distinction from band transport present in most inorganic semiconductors. Conducting polymers display a very different band transport, since the transport of charge occurs in mid-band gap states instead of the regular conduction or valance bands. These mid-band charge carriers are caused by many well known quasi-particles (Solitons, Polarons and Bipolarons) instead of the regular electrons or holes [12]. As these quasi-particles are outside of the scope of this thesis, they will not be discussed with any further detail.

\subsection{Application}

As mentioned earlier in this chapter, the PEDOT:PSS are effectively p-doped by the creation of a hole in the thiophene groups ionically bonded to the negative charge in the PSS backbone chain. In turn, if these charges were to be counter-doped, these ionic bonds would break and the conductivity of the device would decrease. This is the base operation of the optoelectrical bR/PEDOT:PSS device studied in this thesis.

By orientating the bR molecules to have their EP side (discussed later in this thesis) pump ions into the PEDOT:PSS area they cover, the protons will combine with the negative charge in the PSS backbone, which in turn will cause the hole in the EDOT monomer to de-dope, causing an ionic bond to no longer exist between the PEDOT and PSS, which in turn decreases the conductivity of the device. 


\section{Chapter 3}

\section{Literature Review}

This chapter will cover a review of papers and patents related to device covered in this thesis.

\section{1 bR Potential Applications}

Bacteriorhodopsin (bR) has started to spark a lot of interest in various applications, especially in the field of image sensing, as regular CCD and CMOS image sensors still cannot mimic the information processing capabilities found in their biological counterparts. Natural vision seems to offer the highest form of integrated image sensing and signal processing achievable: the information processing begins at the retinal level, whereas artificial imaging relies on analog to digital signal conditioning, resulting in loss of definition and detail. Biological photoreceptors offer the advantage of being fairly simple, display inherent pre-processing capabilities, are more energy efficient, lower cost and can be constructed on flexible substrates. This last part is especially important, as this could mean creation of innovative radial biological photoreceptor arrays which are not bound to planar, single sized, rectangular sensor arrays.

Wang et al. [17] proposed such an implementation which uses the well known Reichardt's delay-and-correlate algorithm for motion detection. By depositing a 16pixel array of bR thin films on a flexible PET substrate (Figure 3.1) and connecting it to a high gain switched integrator along with a sample and hold \& hold circuitry, the group was able to implement a photoreceptor which detected the motion of a probing laser along a row of pixels. This testing methodology will be similar to the one used in this thesis, which uses a probe laser to promote the ion pumping and creates a 
measurable photocurrent.

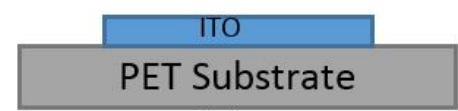

(a)

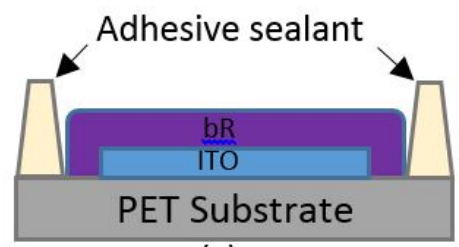

(c)

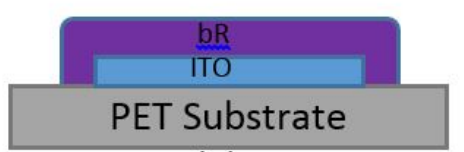

(b)

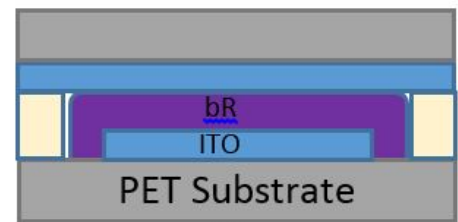

(d)

Figure 3.1: bR based photosensitive pixel [17]

The group also proposed a potential circuit model for bR (Figure 3.2) which could help in future applications where bR must be interfaced with circuitry. Here they estimated the bR molecule as a current generator with a finite resistance of the protein medium $\left(R_{s}\right)$ which can be modeled as a current source $E_{p h}(\mathrm{t})$. The lipids in the membrane will exhibit a high dielectric constant which was modeled as a combination of series capacitor $C_{l}$ and series resistor $R_{l}$. Finally, the parallel resistor $R_{m}$ and capacitor $C_{m}$ represent the behaviour of the non-illuminated bR molecules as well as the lipids which are perpendicular to the bR orientation.

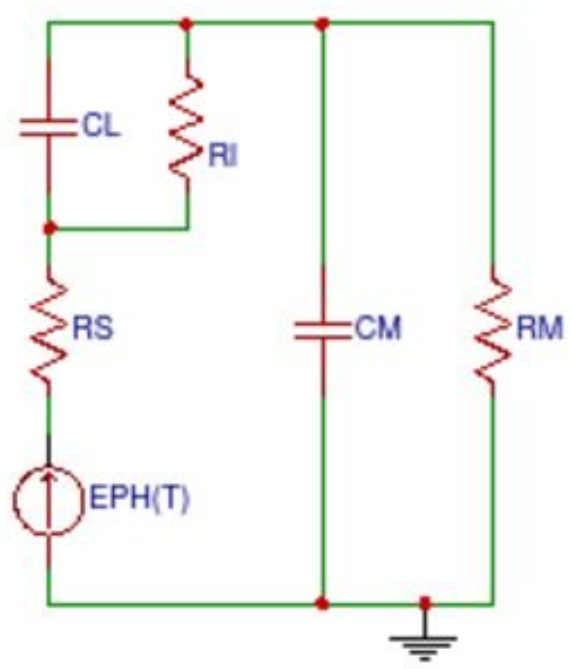

Figure 3.2: Proposed circuit model of bR [17] 
By converting the raw bR output into a digital PWM signal, Wang et al shows that even in its current state bR could be a potentially interesting material for photosensing. Of even more interest is the proposed radial imaging system based on the same pixel array, but taking advantage of the flexible nature of the substrate. Having a flexible substrate brings about possibilities of having a true 360 degree sensing array, or one which can be folded up and moved to different locations with ease. The former being an extremely interesting new concept, as the alternatives in today's security cameras that require large field-of-views and omni-directional imaging usually require bulky reflecting surfaces and wide-angle lens assemblies, which really increase their cost and impracticality.

Of similar interest, Frydrycha et al. (2000) [18] proposed a color sensitive retina based off of bR. Here, the group constructed an artificial retina which processes colours in a way very much analogous to the way a human retina will process colour: Through a combination of "cones" and "rods" ganglion cell structures, all of which absorb different wavelengths to process the full visible spectrum [19]. However, if only wildtype bR was to be used, this would not be a very efficient colour recognizing device, as the absorption peak lies around $570 \mathrm{~nm}$. To circumvent this, the group used synthetic retinal analogues of bR through genetic manipulation, namely 4-ketobR and 3,4-dehydro-bR. The absorption peaks of these analogues shift towards 600 $\mathrm{nm}$ and $500 \mathrm{~nm}$ respectively, with 4-keto having a very high absorption in the range from 400-520 nm (Figure 3.3). In future work, more mutants of bR could be added to cover the full visible spectrum.

Various different elements (pixels), each containing one of the bR mutants, were constructed with the $\mathrm{bR}$ processed as thin films. The pixels are composed of gold/bR/ITO on glass, allowing for transparent electrodes which let the light in. The active bR layer (called the photosensitive layer) cannot detect colour by itself, so a processing layer is added to the device. The processing layer then has to be trained to know which excitations represent which colour, this is done using a self-organizing map (SOM) with the output of the photosensitive layer. Even as far as 1999, we can already see the ease of integration of bR into self-teaching neural networks, in this case to recognize colour.

From these two papers, it can be seen that bR has great potential application in the field of optical sensing by demonstrating very analogous response to our eyes, as well as providing a distinct and measurable output signal, of great compatibility as 


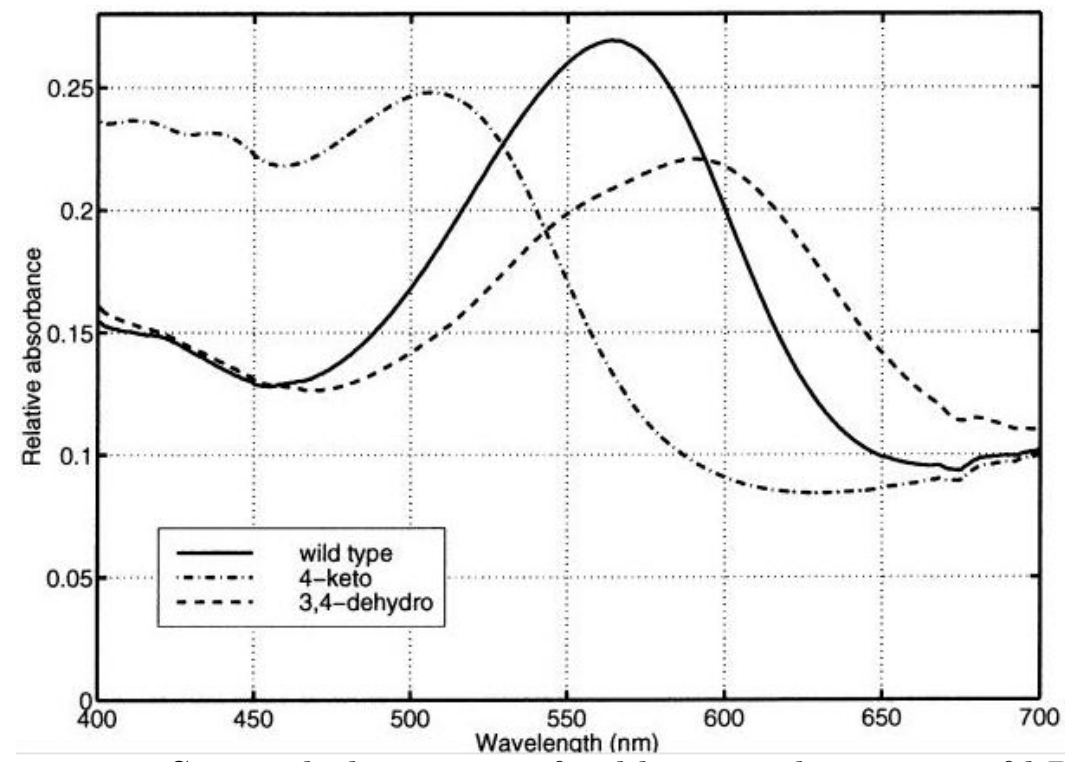

Figure 3.3: Spectral absorption of wildtype and mutants of bR [18]

an input to neural networks and learning algorithms.

Beyond sensing applications, bR has had several proposed applications in the world of biomolecular electronics. With its promise of cost-effective miniaturization, the device integration and optimization possible with bR has been heavily studied. One very interesting and innovative architecture proposed by Birge et al (1999) [20] is a holographic three-dimensional memory. Three dimensional memories are of great interest in the field of computing, as the bottleneck of modern computing has become the transfer rate bandwidth to random access memory. Three dimensional memories offer a large increment in data storage capability, and an optical implementation can offer an even greater push in speeds.

For an optical bR based three dimensional memory, an interesting phenomena of bR is used, called a branch state. The branched state refers to the creating of a long-lived state of bR not normally reached in the regular photoexcitation to thermal decay cycle (bR to $\mathrm{M}$ state and back). This involves photoconversion of the $\mathrm{O}$ state to form a 9-cis chromophore called the $\mathrm{P}$ state, which is only accessible by shinning an $\mathrm{O}$ state bR molecule with red light, which then converts into the unstable chromophore 9-cis $\mathrm{P}$ state $\left(\lambda_{\max }=490 \mathrm{~nm}\right)$, which eventually thermally decays into a stable $\mathrm{Q}$ state $\left(\lambda_{\max }=380 \mathrm{~nm}\right)$. For the purpose of simplicity, $\mathrm{P}$ and $\mathrm{Q}$ are considered as the same state. This $\mathrm{Q}$ state now has the advantage of having a predicted lifetime of 5-20 years (meaning a long time storage), and since the calculated thermal isomerization 
binding barrier is in the order of $4 \mathrm{kcal} / \mathrm{mol}$ (comparable to the activation energy for thermal denaturation of the protein), it is practically impossible for the state to thermally decay back to the bR state. The only way for the molecule to be brought back into its ground bR state is to shine it with blue light [21]. These properties of the protein make it ideal for a memory, as they are binary in nature, with a off state $(\mathrm{bR})$ and a long lived on state $(\mathrm{Q})$. Figure 3.4 shows the use of this new photocycle in the scheme of a writeable memory.

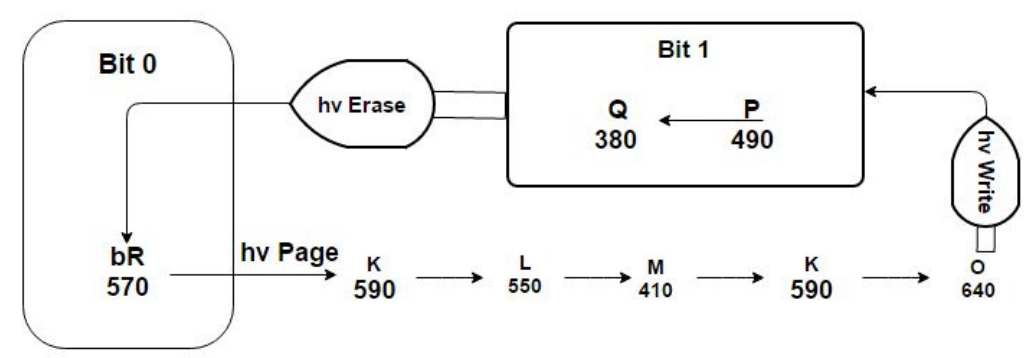

Figure 3.4: Schematic of bR Photocycle memory cycle, with P/O states as the "On" bit and bR state as the "Off" bit

For the creation of such memory, a bR based polymer cuvette was proposed by in situ polymerization of a acrylamide-bR solution. The solution is added to a vacuum degassed fluorescence cuvette. This cuvette now holds the bR matrix which can be used as the memory. To address it (as in standard memories), two active matrix liquid crystal spatial light modulators (AMSLM) can be controlled electronically, one to page thin vertical slices of the bR cuvette and another in a pixel $2 \mathrm{D}$ matrix which can then define areas of bR which will be "written" into the Q state. Reading can be done as well simply by activating all the data SLM pixels, and having a detector CCD array detect the areas which are already in the Q state (as they will not absorb the red laser). A schematic of this cycle can be seen in Figure 3.5.

A full erase can be done by sweeping the paging SLM across the cuvette while using a blue laser. The group was able to implement a 1024x1024 voxel bR cuvette based memory with relatively low bit error, albeit not very rugged to environmental variants like $\mathrm{pH}$ or temperature. However, this does demonstrate a very promising usage of biomolecular electronics.

Other applications which have been demonstrated include solid-state optical switches [22], treatment of eye disorders such as macular degeneration [23], and a 


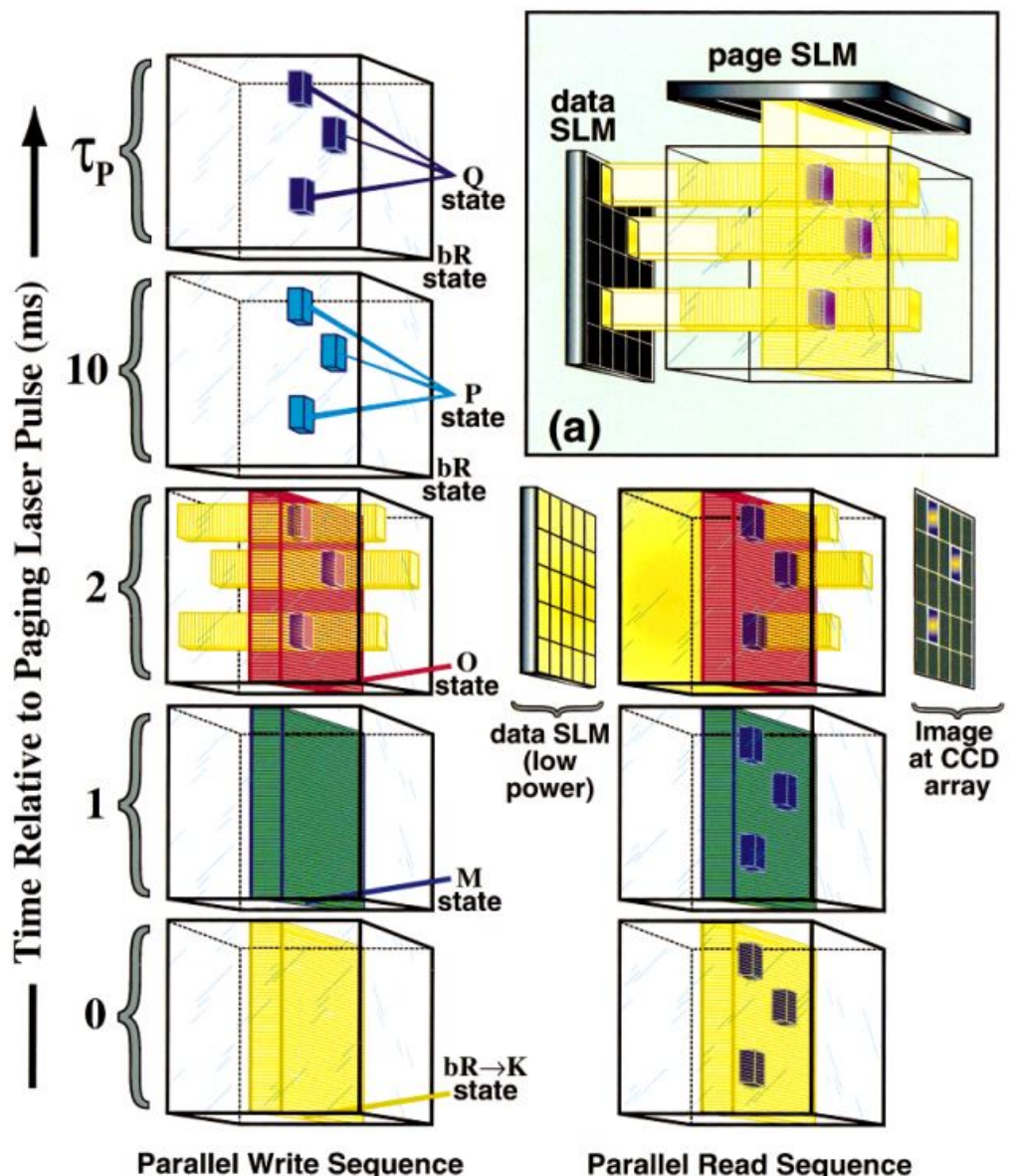

Figure 3.5: Parallel write (left) and read (right) sequence of 3 bits of data using the branched cycle architecture propsed in Figure 3.4 [20]

whole other range of medical and instrumentation/sensing applications.

\subsection{PEDOT:PSS Potential Applications}

Since the discovery of polymeric organic conductors in 1977, these materials have been studied very rigorously to bring the technology into its current state. As previously mentioned in the thesis, the semiconductor properties of conducting polymers are highly sought out for various applications, especially in fields where transparent, flexible conducting materials are required. Various different conducting polymers have been studied for various purposes [24]:

- Polythiophene: offers great environmental and thermal stability, better solubility and higher capacitor behaviour. Applied in charge dissipation coatings 
in electron-beam lithography and an active semiconducting material in organic thin-film transistors.

- Poly(para-phenylene Vinylene) (PPV): high levels of crystallinity, small optical band gap and bright yellow fluorescence make it ideal for uses in organic LEDs and photovoltaic devices. Can also be easily doped, making it easy to change its electrical and physical properties with the inclusion of functional groups.

- Poly(carbazole): used mostly in polymer film electrodes, electrochromic display devices and batteries.

- Polyaniline (PANI): has been polymerized on multiple electrodes, including Platinum, glassy carbon and CFME, due to the good knowledge of the oxidized and reduced states of PANI films. PANI is very popular due to its diverse structure, thermal and radiation stability, low cost, ease of synthesis and conducting properties. Uses include gas sensors and corrosion protective coatings as well as micro-electronics.

- Polypyrrole (PPy): One of the better studied conducting polymers, due to its ease of synthesis, good redox properties, stability in oxidized form, water solubility and ability to have high conductivity. Proposed in several applications such as batteries, supercapacitors, electrochemical biosensors, conductive textiles and fabrics, EMI shielding, drug delivery systems and mechanical actuators.

However, when it comes to the world of electric and electronic applications Poly(3,4-ethylenedioxythiophene)-poly(styrenesulfonate) (PEDOT:PSS) is the most dominant conducting polymer. One of the reasons for this is the potential for PEDOT:PSS to replace indium tin oxide (ITO) as a transparent conductor. PEDOT:PSS is on its way to achieve conductivity on the level of ITO, being almost within an order of magnitude [25], while offering the same transparency at a fraction of the cost, making it a really appealing component to add to many application that require transparent conductive layers.

One of these applications would be as a conductor in a electroluminescent (EL) device. Normally, inorganic electroluminescent devices consist of a composite active layer such as zinc sulfide emitter and a dialectric in between two conducting layers, one of which is transparent. When AC voltage is applied (for $\mathrm{ZnS}: 100 \mathrm{~V}$ @ 400 $\mathrm{Hz}$ ), the $\mathrm{ZnS}$ layer emits light, the colour which can be tuned by different doping 
agents. The transparent layer used normally is ITO, but can be readily replaced with PEDOT:PSS (Figure 3.6) [25]. While the conductivity of ITO is larger than that of PEDOT:PSS, for an EL application this isn't a problem, as the high voltages the devices are operated at, specific resistance in the transparent layer can be relatively low. The major advantage of having a conducting polymer as the transparent layer is that it can be applied by means of silkscreen printing just like the active layers, making processing easy and cheap. ITO normally has to be deposited by sputtering techniques, a much more expensive process. Another major advantage is that this allows for flexible EL devices, since PEDOT:PSS does not suffer form the brittleness of ITO.

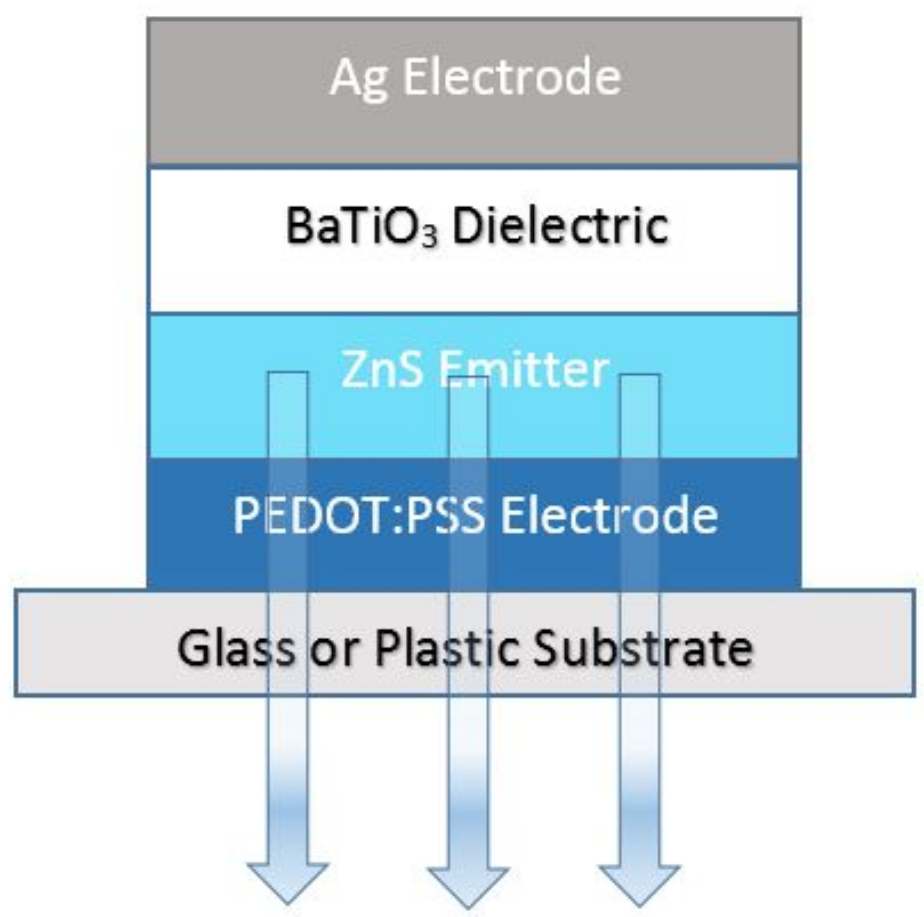

Figure 3.6: EL Device with PEDOT:PSS as a transparent electrode

Along the lines of the functionality in EL devices, PEDOT has also found a usage within the field of Organic Light Emitting Diodes (OLEDs) [25]. Here, PEDOT:PSS can be used as the hole injection layer. OLEDs operate by having an organic emissive layer (EML) placed either between and electron injection layer (EIL) and a hole injection layer (HIL), or just one of them, all sandwiched between a anode and a cathode. When the anode is placed at a higher potential, hole injection occurs form the anode to the HOMO of the HIL, and electrons are injected from the cathode into 
the LUMO of the EIL. Holes and electrons migrate towards the EIL where the charges will localize on the same molecule due to spacial proximity, causing a fraction of the to recombine and form an exciton (a localized electron-hole pair having an excited energy state). These excitons will then relax and decay back to their ground state, where they will recombine, which causes energy to be released as light of a wavelength determined by the EML material. This light will be perpendicular to the substrate, and as such one of the electrodes and transport layers must be transparent.

Not only is the addition of a cheap, flexible transparent layer interesting in the scope of OLEDs, but also of interest is the addition and modification of the PEDOT:PSS layer for enhanced device performance. Xiao et al. (2012) [26] demonstrated that the additions of Gold nanoparticles (Au NPs) could increase the luminosity efficiency of the device. A device was created with a PEDOT:PSS:Au NP mix as the HIL. An ITO/PEDOT:PSS:Au NPs/EML/ $A l q_{3} / \mathrm{Al}$ device was fabricated (Figure 3.7), using very standard commercial fabrication techniques for OLEDs. The Addition of Au NPs did not change the thickness of the the PEDOT:PSS layer, as the concentration of the NPs is so small. However, this small concentration showed an improvement in green emission luminescence efficiency of almost $1 \mathrm{Cd} / \mathrm{A}$ in, while still maintaining almost the same current density to voltage response.

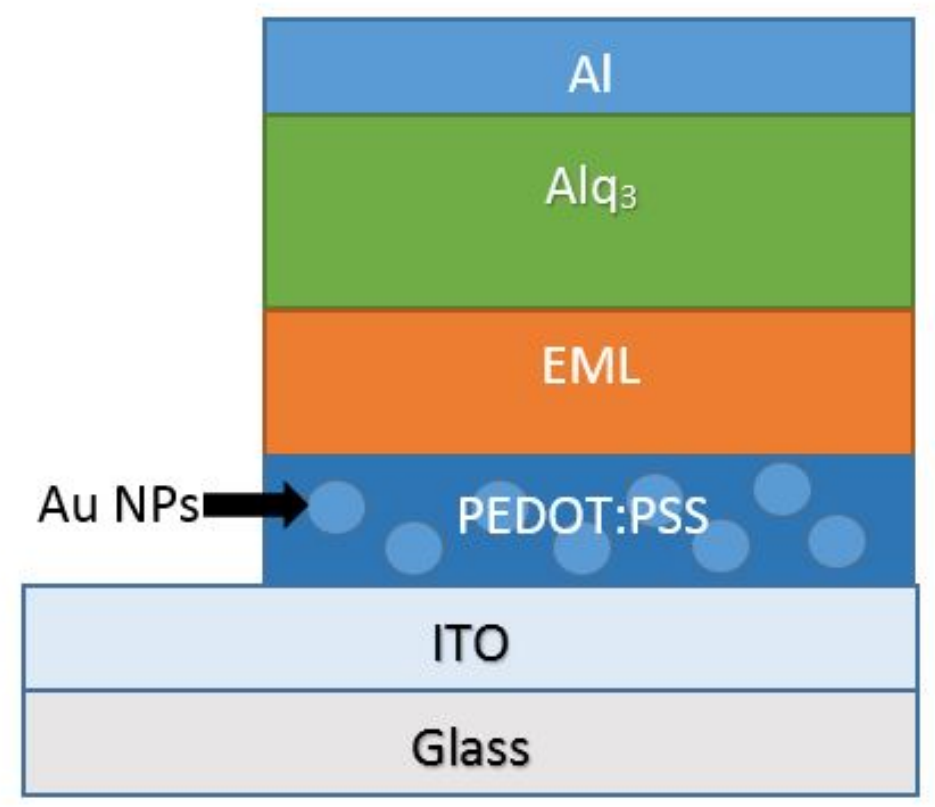

Figure 3.7: OLED Stack with a PEDOT:PSS/AU NPs HIL 
In another paper, Wang et al. (2008) [17] proposed the introduction of multi wall carbon nanotubes (MWCNT) into PEDOT:PSS nanocomposites to improve the response of the OLEDs. Since both materials display p-type semiconductor properties as well as high conductivities, creating a nanocomoposite that maintains the optical transparency of pristine PEDOT:PSS proves a great solution for increasing the response of the device. For this device, a concentration of 0.4 wt.\% acid treated MWCNT were added to a high conductivity PEDOT:PSS solution and spin coated on a ITO coated PET substrate. The group created a ITO/PEDOT:PSS: wt $0.4 \% \mathrm{MWCNTs} / \mathrm{EML} / \mathrm{Alq}_{3} / \mathrm{Al} \mathrm{OLED}$, and compared it to a ITO/EML $/ \mathrm{Alq}_{3} / \mathrm{Al}$ and ITO/PEDOT:PSS/EML/ $\mathrm{Al}_{3} / \mathrm{Al}$ devices. The nanocomposite device showed much higher current densities for a lower voltage $(10-50 \mathrm{~mA} / \mathrm{cm})$ as well as much higher luminance $\left(1000-6000 \mathrm{~cd} / \mathrm{m}^{2}\right)$. These two papers show that there is a very large room for improvement in the field of nanostructuring to create even more efficient OLEDs.

Of very similar interest as well is the nanostructuring of PEDOT:PSS as a hole transport layer in photovoltaic applications. Here, a photon striking a semiconducting material will generate an electron-hole pair, so having a highly conductive layer ptype layer to transport the charge to the anode is highly sought after. Xia et al (2011) [27] demonstrated how appropriate choices of organic cosolvents could heavily improve the conductivity of PEDOT:PSS and increase the efficiency of these solar cells in which the treated PEDOT:PSS was used as the hole transport layer.

Other applications of PEDOT:PSS have been found in electrolytic layers for super capacitors [28], conducting layers for printed wiring board manufacturing [25], electrically conducting coatings, conductive layers in all organic thin film transistors [24].

\subsection{PEDOT:PSS/bR Device Potential Applica- tions}

While extensive research has been done for both materials previously mentioned, not a lot of work has been done in the interfacing of these two materials. As mentioned previously in chapter 2 , bR and PEDOT:PSS seem like they would have very complementary functions and could be used for various optoelectrical sensing applications. Some work has been put into the characterization of such devices, mostly in terms of the voltage controlled photoresponse of the device. 
Arun and Narayan (2007) [29] explored a very similar architecture to this thesis, where they created a device in the hopes of using a conducting polymer as an antenna for probing biophysical activities. Two conducting polymers, PEDOT:PSS and PANI:DBSA (Polyalianine doped with dodecylbenzene sulfonic acid) were laid down in a strip over gold $(\mathrm{Au})$ contacts, and a drop of bR was dried over the strip conducting polymer and let dry under an external electric field (explained later in this thesis). The bR was the illuminated with a green laser (peak bR absorption wavelength) modulated between with a chopper at 4-400 $\mathrm{Hz}$ and fed into a lock in amplifier, with the resulting photoelectric response between the Au nodes was then plotted as a function of time. Here they were able to reproduce the very characteristic transient signal/spike bR response, with a large positive spike $(\sim 0.35 \mathrm{mV})$ when turning on the probe laser, a slow increase in voltage as the bR molecules start pumping more ions, and a large negative spike $(\sim 0.25 \mathrm{mV})$ when the laser is turned off, representing the re-uptake of protons.

Using all these results and approximations, the group estimated the spectral and temporal response of the bR could be modelled using a two-level kinetic model, consisting of the ground state (bR) and intermediate state (M), with the total molecules $N_{T}(t)=N_{B}(t)+N_{M}(t)$, where at time $\mathrm{t}$, these represent the number total number of molecules, the number of molecules in bR state, and the number of molecules in the $\mathrm{M}$ state, respectively. The forward rate of the reaction from bR to M state can be called $k_{B}$ and the rate of backward reaction being limited by the lifetime of the M state, labeled as $\tau_{M}$. Furthermore, a simple first-order reaction which models the number of molecules at time $t$ in the ground state after photoexcitation was derived:

$$
N_{B}(t)=\frac{N_{T}}{k_{B}+\frac{1}{\tau_{M}}}\left(\frac{1}{\tau_{M}}+k_{B} \cdot e^{-\left(k_{B}+\left(1 / \tau_{M}\right) \cdot t\right.}\right)
$$

Where at time $\mathrm{t}=0, N_{M}=0$ and $N_{T}=N_{B}$. Once the light is turned off, the M state thermal decay to its initial bR ground state can be modelled as:

$$
N_{M}(t)=N_{M}(S S) e^{\frac{-t}{\tau_{M}}}
$$

Where $N_{M}(S S)$ is the wavelength and intensity-dependant steady state distribution of M-state molecules.

A 2nd form of this architecture was created by having an aluminium electrode being thermally evaporated over the bR patch, almost like a gate to a transistor. 
They measured the photoelectric current response across the lateral Au electrodes with the Al gate floating, and it displayed the similar transient bR signal as discussed above. Moreover, now the group could use the $\mathrm{Al}$ electrode to probe the transverse process across the bR-conducting polymer interface, since now you can measure signals from the $\mathrm{Al}$ electrode and the Au electrodes shorted together. Also, by increasing the voltage added to the top Al layer, the current response of the was greatly increased in magnitude $(\sim 0.28 \mathrm{nA})$ because of the contribution of the electric field. Manoj and Narayan (2003) [30] proposed a "sandwich" configuration where the bR/PEDOT:PSS device is sandwiched in between an ITO and Al electrodes, an architecture much more similar to the device mentioned above, which both display similar amplitudes of photoresponse $(\sim 0.3 \mathrm{nA})$, a much larger response than for the purely lateral device $(\sim 0.8 \mathrm{pA})$. These studies not only give insight into the charge transport of the bR/PEDOT:PSS device, but give hope to further tune, enhance and modulate the signal for different applications.

Arun and Narayan then decided to study the effect of the ion motion across the interface on resistivity. To do so, they monitor the lateral conductance current $I(t)_{A u-A u}$ of the polymer under a constant lateral bias $V_{A u-A u}=0.5 \mathrm{~V}$. As a control test, a voltage was applied to the top $\mathrm{Al}$ electrode without any photoexcitation and the photocurrent was observed, yielding no change (ie. no de-doping of the conducting polymer). Upon photoexcitation, a increase in resistivity of the polymer layer could be seen directly associated with the increment in Al voltage, which did not persist upon the removal of photoexcitation. Once the probing laser was removed, the conductivity of the PEDOT:PSS layer decayed back to its original value at a rate modelled by an exponential decay in terms of the diffusion of the protons in bR.

From these observations, a speculation as to the characteristic spike behaviour seen in the PEDOT:PSS lateral layer was concluded to arise from the change of the dipole moment within the adjacent bR layer upon photexcitaion. This dipole moment change induces an electrical field which modifies the interface potential between the $\mathrm{bR}$ and the PEODT:PSS, which in turn de-dope the PEDOT:PSS and change the surface charge transport pathways, giving that characteristic "spike" in conductivity. The ion exchange will eventually reach an equilibrium with the chemical charge gradient, at which point the response will stay at a steady voltage until the light is turned off, in which case the re-uptake of ions by the bR layer will cause the characteristic reverse spike. 
As far as potential applications that could become a product, Takamatsu et al (2008) [31] developed a bendable touch screen interface based on bR and PEDOT:PSS. Since normal touch screen work based on applied pressure to two transparent electrodes to change the capacitance between them, any mechanical deformation would result in changes in the gap between electrodes making it not suitable for the application of flexible displays/touch screens. This is where the integration of a bR/PEDOT:PSS sensing element for the flexible touch screen comes in.

To create a pixel (Figure 3.8) for this device, a solution that blends both bR and PEDOT:PSS is dispersed as a thin film on a PET substrate. Polydimetylsiloxane (PDMS) is then used to define an area which will be a reservoir for an electrolytic buffer solution (potassium chloride) between the organic layer and the Au counterelectrodes, which will be used as a proton donor for the pumping mechanism of bR. The basic idea of the device is that a light pen (containing a green laser or LED) will shine upon the bR/PEDOT:PSS elctrode, causing the de-doping of the PEDOT:PSS through the regular photoexcitation process. Through the characteristic "on" positive spike response between the PEDOT:PSS and Au electrodes, the system can detect when the light is being shone upon the pixel, and the characteristic "off" negative spike when then light is removed.

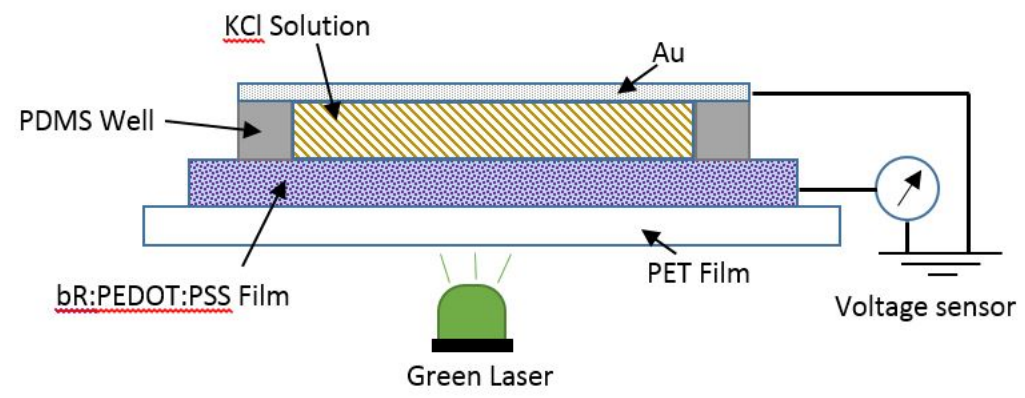

Figure 3.8: Flexible touchscreen pixel [31]

A 4x4 pixel display was created, with each pixel connected to a zero shunt current amplifier with its output connected to a low pass filter with a gain of $40 \mathrm{~dB}$ and $100 \mathrm{~Hz}$ cut-off, which were then connected to 16-bit A/D board where the output voltage from a pixel was measured by the relationship between the photocurrent and intensity, and then digitized into a computer. The display was then tested by drawing some shapes on it, including a transverse line, a box and a $\mathrm{H}$, all of which produced the correct output in the computer. Finally, the same measurements were taken with 
the pixel display being bent, and showing that both the photocurrent and absorption remain similar even under deformation.

Since minimal work has been done in the field of characterization of a bR/PEDOT:PSS interfaced device, this thesis wanted to probe further into this topic, which will hopefully help understand the optical and electrical response of such a device for future research. 


\section{Chapter 4}

\section{Design And Fabrication}

\subsection{Introduction}

This chapter will deal with the processing steps required for the formation of PEDOT:PSS-bR devices. For the purpose of this device, spincoating was used as the main deposition step for PEDOT:PSS, as very thin film (around $100 \mathrm{~nm}$ ) were needed since bR can only provide a limited amount of protons to de-dope the conducting polymer. Also for simplicity, reactive ion etching was used to define the device areas, as this meant there was no need to use under-studied wet processing steps which include various chemicals that can affect the PEDOT:PSS films. However, other forms of PEDOT:PSS processing were investigated, including lithography, lift-off and different types of gases for plasma etching.

\subsubsection{Device Design}

The first step to create the device was to design the architecture which it would use. The wafer selected was silicon with an insulating layer covering it, as to electrically isolate the conducting polymer layer, which is the only place where current will be flowing. Silicon was also selected because processing on this as a substrate can give insight into the integration of the process into modern semiconductor processes if this material was to ever be used in conjunction with modern CMOS technologies.

The stack of the device would consist of the PEDOT:PSS on top of the insulating layer and the bR layer on top of that. This bR layer would then have to be oriented for an increase in device performance. To be able to electrically contact the PEDOT:PSS layer, Silver contacts have to be used as the material is otherwise too soft to be probed 
with metal tips, which will just pierce the material and contact the insulating layer below it. A high level process schematic can be seen in Figure 4.1 below

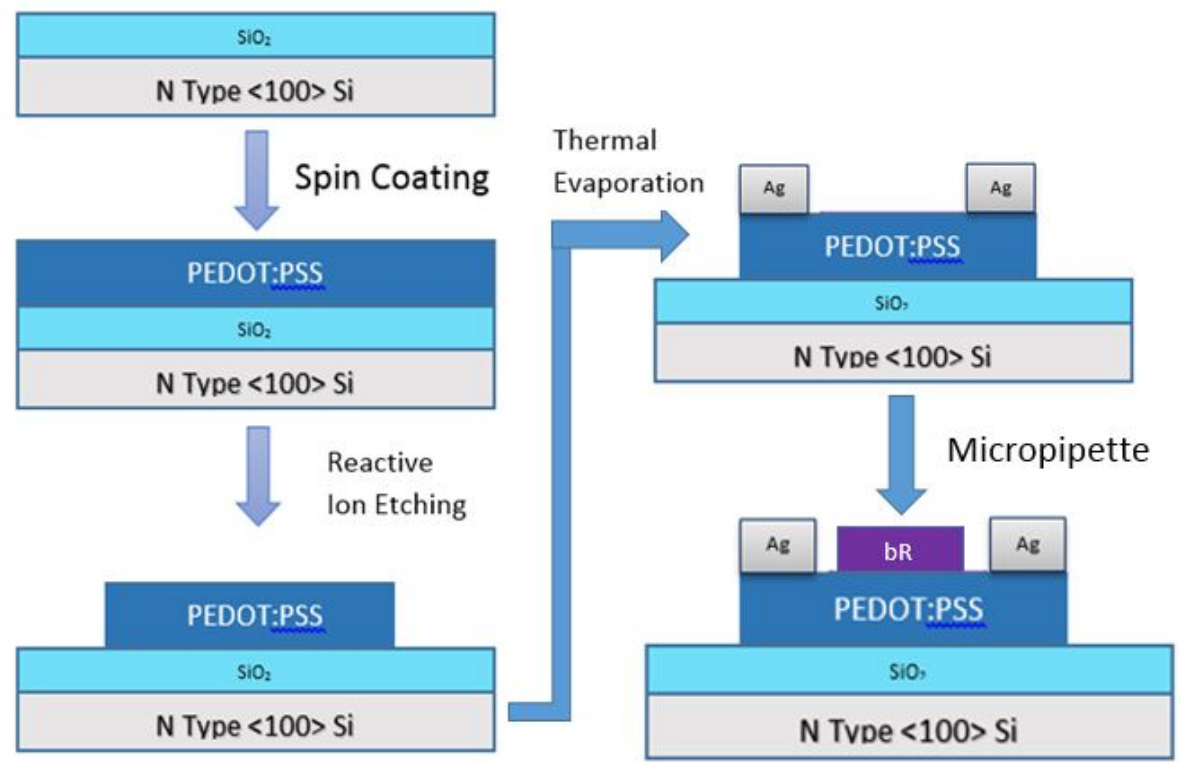

Figure 4.1: High level architecture of the bR/PEDOT:PSS device

This chapter will overview the different processing methods to fabricate this device.

\subsubsection{Mask Design}

Because reactive ion etching will be used for etching, a shadow mask had to be designed to define the device areas. One of the main considerations of the shadow mask design was to be able to have various feature sizes to be able to study the effect of device size on the response. Another consideration was to have a large amount of devices so that once spinning and etching was done, there could be an array of different devices to chose from if there were any faults in the film. This of course made the devices more susceptible to process variation, but this was a trade-off for the flexibility of selecting the best devices.

To create the masks, L-Edit 2016.1 was used. Two masks had to be created, one which would define the area in which the PEDOT:PSS device was going to be, and another one which will define the area of the metal contacts.

The first mask designed was the one which would define the device region. To do so, device size had to be first considered. At the time of design, rectangular devices 
were chosen, since they allow for an easier dispersion of the bR and allow to see the effect of the distance between the device active region (where the probing laser hits the bR/PEDOT interface) and the bond pads. Another consideration was to have all devices be the same amount of squares, which would allow for all the devices to have consistently similar resistances.

The final sizes of the devices and the number of devices can be seen in Table 4.1. Figure 4.2 (a) shows the design of the mask used for defining the device area when etching the substrate, and Figure 4.2 (b) shows the design of the mask used to define area of the metal contacts.

Table 4.1: Number of devices

\begin{tabular}{||cccc||}
\hline Device & Device Size $(\mu \mathrm{m})$ & \# of Vertical & \# of Horizontal \\
\hline \hline 1 & $250 \times 1000$ & 143 & 156 \\
\hline 2 & $1000 \times 4000$ & 24 & 25 \\
\hline 3 & $2000 \times 8000$ & 3 & 3 \\
\hline 4 & $3000 \times 12000$ & 0 & 2 \\
\hline 5 & $4000 \times 16000$ & 3 & 3 \\
\hline
\end{tabular}

As seen in Figure 4.2 (a) below, the smaller devices could be set in a grid to maximize the area and increase the overall number of devices. The crosses on the left and top side were used as alignment guides.

The 2nd mask that was created seen in Figure 4.2 (b) was to define the areas where the metal contacts will be. Since metal evaporation (see section below) was to be used, this mask protected the devices and let the metal vapour contact the PEDOT:PSS to form the bond pads.

The sizing of the pads can be seen in Table 4.2 below.

\subsection{PEDOT:PSS Spin Coating}

The first method of of PEDOT:PSS deposition for the purpose of an optoelectronic device was to use spin coating. With spin coating, the aqueous dispersion of PEDOT:PSS could be easily controlled to the desired film thickness. Elschner et. al [12], 


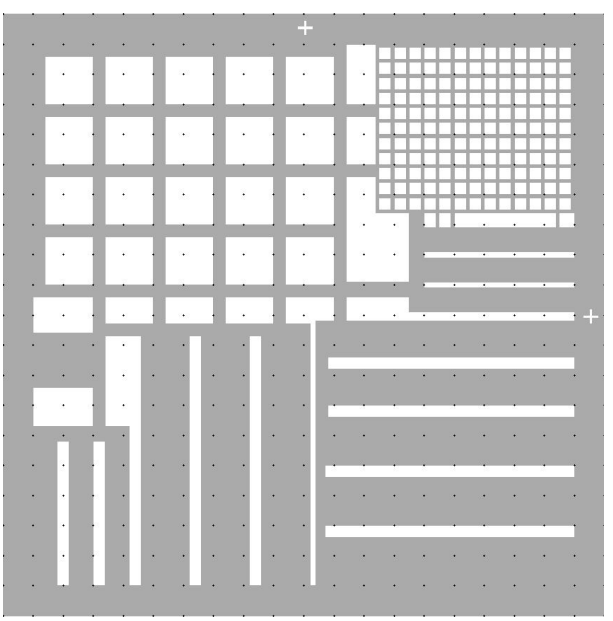

(a)

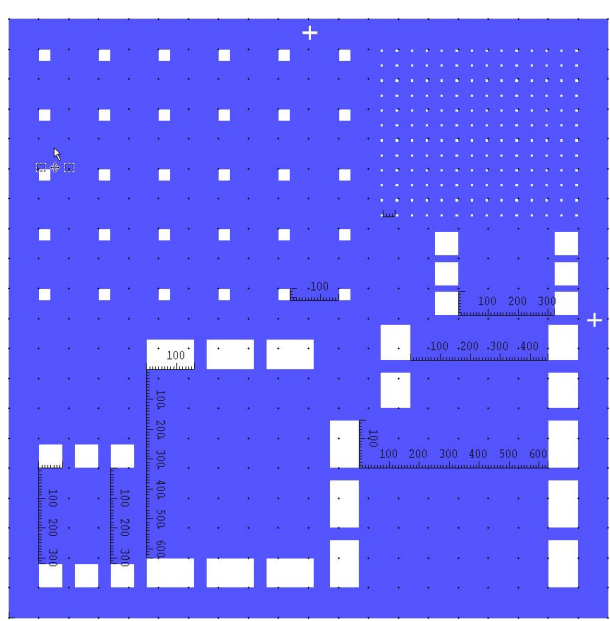

(b)

Figure 4.2: Design of (a) device defining mask and (b) contact defining mask

Table 4.2: Size of Pads

\begin{tabular}{||cc||}
\hline Device (from table 1) & Pad Size $(\mu \mathrm{m})$ \\
\hline \hline 1 & $250 \times 250$ \\
\hline 2 & $1000 \times 1000$ \\
\hline 3 & $2000 \times 3000$ \\
\hline 4 & $2500 \times 3000$ \\
\hline 5 & $2500 \times 4000$ \\
\hline
\end{tabular}

amongst many others, has studied and plotted the effect of spin speed on film thickness from which it was interpolated that speeds of around 1000 RPM will yield layers of about $100 \mathrm{~nm}$ for low viscosity, low conductivity dispersion. For the purpose of this thesis' device, the substrate used was a N-type $<100>$ Silicon (Si) wafer with $1000 \stackrel{A}{A}$ Silicon Dioxide $\left(\mathrm{SiO}_{2}\right)$ insulation layer grown on the surface. This is due to the device being a lateral conductivity device where the conduction only happens in the PEDOT:PSS layer, and thus if no $\mathrm{SiO}_{2}$ were to be present, the Si would operate as an electrode to PEDOT:PSS and disperse the charge.

From literature, it was gathered that the first step of spin casting any PEDOT:PSS films was the cleaning and condition the substrate. Substrates were cleaned using a acetone, DI water and propanol solution in ultrasonic bath, as to remove all possible contaminants from the $\mathrm{SiO}_{2}$ surface. For the conditioning of the wafer surface, a treatment of 5 minutes in oxygen $\left(\mathrm{O}_{2}\right)$ plasma, which helps to both descum the surface 
wafer from any organic material particles, as well as change the surface energy of the $\mathrm{SiO}_{2}$ and increases hydrophilicity, which increases the chances of adhesion [32].

One of the main challenges with PEDOT:PSS films is its unwillingness to stick to the substrate. Although $\mathrm{SiO}_{2}$ is hydrophilic, various attempts to spin coat commercial grade Baytron P (labelled at $1 \mathrm{~S}-\mathrm{cm}$ ) colloidal solution proved unsuccessful. One of the methods of improving the adhesion was to introduce surfactants to the Bayton $\mathrm{P}$ to increase its wetability. The simplest surfactant to be used was isopropyl alcohol (IPA) Since IPA will reduce the surface tension of the mixture, causing the dispersion to spread on the wafer at a smaller wetting angle, hopefully improving wetting of the surface during spinning, leaving a uniform PEDOT:PSS film. A solution of 90\%:10\% (w/w) Baytron:IPA was mixed and placed in an ultrasonic bath, since the obtained dispersion is quite old and the polymeric chains tend to "coil up", lowering conductivity and ultrasonication can help unwind these coils [12]. To dispense the solution, it was passed through a $0.45 \mu \mathrm{m}$ PVDF hydrophilic filter, as the average particle size of the PEDOT:PSS can range from $10 \mathrm{~nm}$ to $1 \mu \mathrm{m}$, so a smaller $0.22 \mu \mathrm{m}$ filter may filter out too many conducting particles. After Oxygen plasma treatment, the wafer was blown with Nitrogen $\left(N_{2}\right)$ gas to rid the surface of any particles, and placed in the spin coater. It was found that for this mixture, dynamic dispensing (dispensing while the chuck is spinning) yielded better results, and so $5 \mathrm{~mL}$ of the Baytron:IPA mixture was dispensed onto a wafer spinning at $500 \mathrm{rpm}$ for 15 seconds to wet the whole surface. The chuck then accelerated to $1000 \mathrm{rpm}$ for 60 seconds. Finally, the wafer was placed in a hot plate to thermally anneal, starting at $60^{\circ} \mathrm{C}$ and ramping up to $120^{\circ} \mathrm{C}$ in 1 hour, as to allow for the moisture to slowly evaporate off of the surface, creating a more uniform film which may be able to cover up some of the impurities. This method yielded fairly uniform films of $100 \mathrm{~nm}$, but all films seemed to exhibit some sort of visible particles on them. In theory the filter and $N_{2}$ blow should have gotten rid of these, but no matter what precautions were taken there was never perfect uniformity in the films.

A Plasmos Ellipsometer was used to measure the thickness of the device. Ellipsometry is an optical technique that measures the change in polarization of an incident laser upon reflection or transmission [33]. Scans were taken at 9 different points on the wafer as seen in Figure 4.3 to study the thickness and uniformity of the film, with results in Table 4.3. 


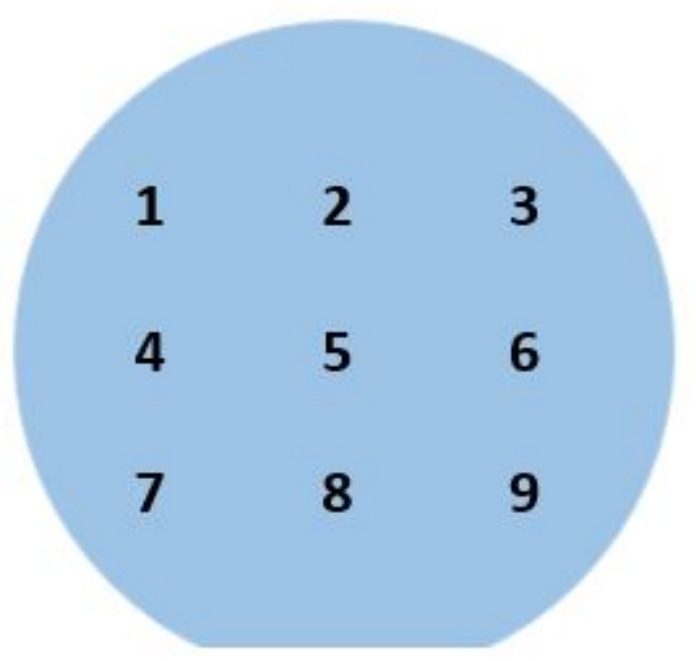

Figure 4.3: Areas of Ellipsometry Measurement

\begin{tabular}{||cc||}
\hline Area & Thickness $(\stackrel{\circ}{A})$ \\
\hline \hline 1 & 919.32 \\
\hline 2 & 935.15 \\
\hline 3 & 907.83 \\
\hline 4 & 1029.29 \\
\hline 5 & 1185.65 \\
\hline 6 & 956.55 \\
\hline 7 & 986.63 \\
\hline 8 & 1046.04 \\
\hline 9 & 1163.70 \\
\hline
\end{tabular}

Table 4.3: Table of Ellipsometry Results

The wafer is fairly uniform, showing an expected Gaussian distribution with thicker layers in the middle of the wafer, and thinning out as it approaches the edges, where the centrifugal force is pushing the material off.

Another commercial grade form of PEDOT:PSS called Orgacon EL-P3040 (680 $\Omega / \square)$ was used. This dispersion is normally used for screen printing, and as such displayed much larger viscosity (>8 Pas), meaning that diluting down would result in a solution watery enough to properly wet the substrate, but viscous enough to have good adhesion. To do this IPA was added to the mix in a ratio of 3:1 PEDOT:PSS to IPA, which noticeably decreased the viscosity of the EL-P3040. Similar steps were taken with the Baytron P dispersion, except that for the EL-P3040, static dispensing where the whole surface was covered before spinning seemed to yield better results. Fairly uniform films were produced, although surface grain size seemed to be increased, and the conductivity of this dispersion proved too high for the application of this optoelectronic device, since lower conductivities would guarantee a larger, more noticeable change in resistance. 


\subsection{Reactive Ion Etching}

To define the active regions of the device, sections of PEDOT:PSS had to be isolated. One of the simplest and quickest methods to do so was reactive ion etching, since this is a dry etching technique, which would compromise the PEDOT:PSS the least as the material is highly sensitive to moisture and dissolves in water. To be able to define the areas for the device, a shadow mask which covered the PEDOT:PSS active regions was to be designed. These would protect the area of the device while the plasma etching removes the unprotected areas. Different gases with different etch rates were tested as well, as described below.

\subsubsection{Gas Etch Rate}

Reactive ion etching uses chemically reactive plasma to remove material deposited on wafers. For the purpose of removing organic material, the March RIE Jupiter II was used, and two different gases were studied. Charlot et al. (2013) [34] studied the effect of $\mathrm{O}_{2}$ and $\mathrm{CF}_{4}$ as the etching gases. $\mathrm{O}_{2}$ seemed to demonstrate a inconsistent etching rate, starting at $400 \mathrm{~nm} / \mathrm{min}$ and winding down to $70 \mathrm{~nm} / \mathrm{min}$, with the $C F_{4}$ gas having a constant etch rate. However, Khaldi et al. (2011) [35] demonstrated that a combination of $90 / 10 \% O_{2} / C F_{4}$ gas yielded the highest etch rate of $1.95 \mu \mathrm{m} / \mathrm{min}$. For this application, a higher etch rate was highly desired because the of the use of a shadow mask.

To place the mask and define the protected area, the 2 inch wafer with PEDOT:PSS would be placed over a larger 4 inch $\mathrm{Si}$ wafer, the mask is placed over it and then taped down the edges with Kapton tape, as seen in Figure 4.4. The shadow mask physically covers the PEDOT:PSS layer, meaning that there isn't a perfect contact between the mask and the layer. Because the mask is a thin metal and it doesn't perfectly rest over a rounded wafer, there was some bending once the mask is taped down. Although the March RIE provided a highly directional etching profile, the bending in the mask still allowed for gas to get underneath and etch the desired active areas, albeit at a slower rate. Because of this, the high etch rate was desired as to leave the desired area intact.

To etch the PEDOT:PSS layer, wafer was placed in the March RIE, the gas flow rate of $\mathrm{O}_{2}$ gas was set to $270 \mathrm{sccm}$, and the rate of a mixture of $\mathrm{CF}_{4} / \mathrm{O}_{2}$ gas was set 


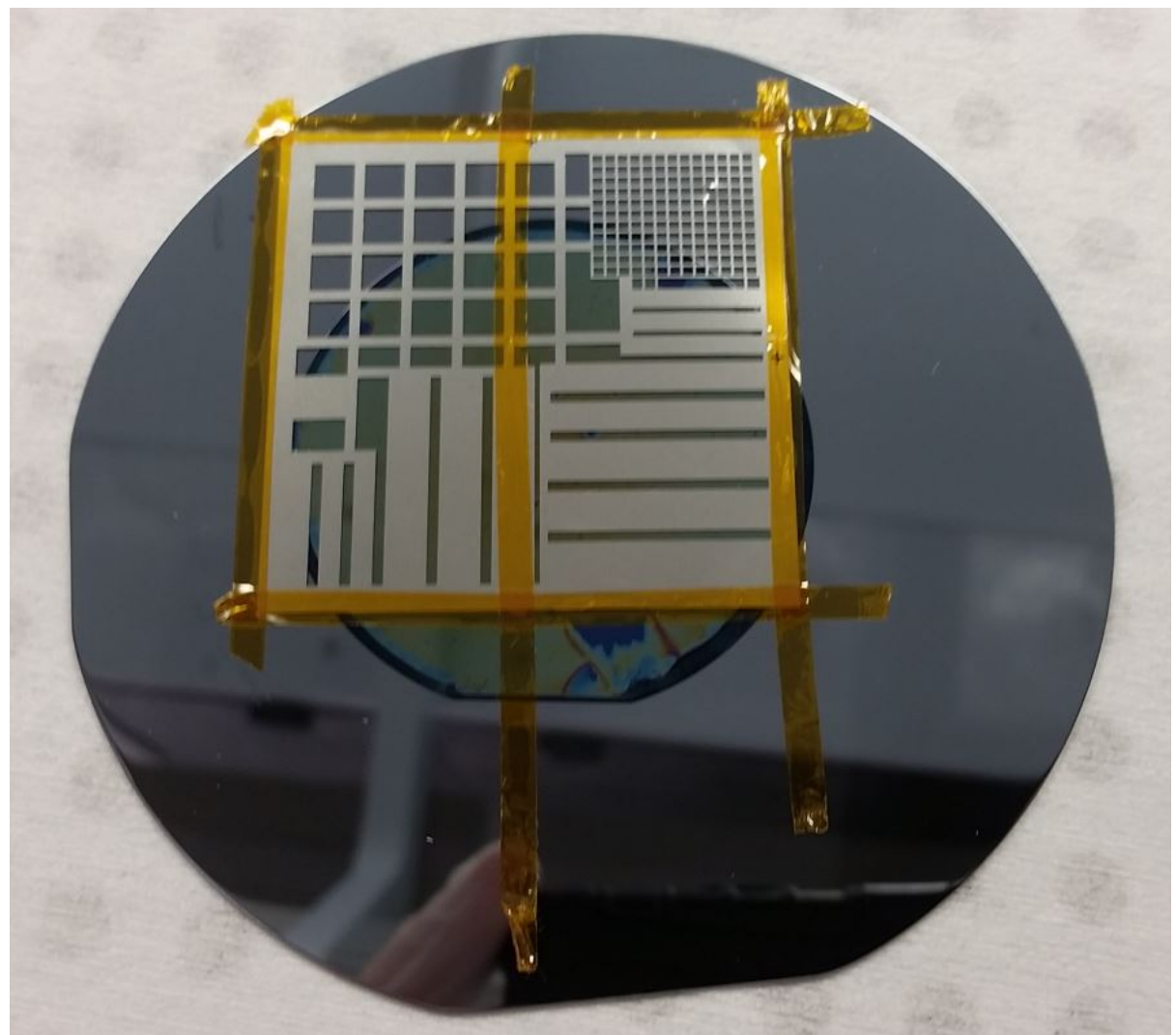

Figure 4.4: Wafer with mask ready for etching

to $28 \mathrm{sccm}$, yielding the total desired mixture of $90 / 10 \% \mathrm{O}_{2} / C F_{4}$. The power was set to 200 Watts and etching time set to 20 seconds. As mentioned above, the high power, low time, fast etching conditions were the most desired etching conditions for this application, as they set the conditions for the least amount of compromise of the conducting film which will act as the active area of the device.

Table 4.3 shows the processing steps to spin coat and pattern a Bayron P PEDOT:PSS device on a silicon wafer:

\subsection{Lift-off}

Although wet processing of PEDOT:PSS is normally avoided, this will not necessarily be viable in a commercial scale process, where the material will have to be integrated into a process that will most likely have some wet processing step along the way. Because of this, we also tried to investigate the effects and results of doing wet processing on a PEDOT:PSS film. Ouyang et al. (2014) [36] proposed a process for PEDOT:PSS 
Table 4.4: PEDOT:PSS Spin Casting processing

\begin{tabular}{||ccl||}
\hline Step & Description & \multicolumn{1}{c||}{ Details } \\
\hline \hline 1 & Wafer Conditioning & $O_{2}$ plasma, 5 minutes \\
\hline 2 & PEDOT:PSS Deposition & $\begin{array}{l}\text { Baytron P:IPA 90\%:10\% Dilution, spin coated at } \\
500 \text { RPM for } 15 \mathrm{~s}, 1000 \text { RPM for } 60 \mathrm{~s}, \text { dynamic dis- } \\
\text { persion }\end{array}$ \\
\hline 3 & Hard Bake & $\begin{array}{l}\text { Hot plate, Start at } 60^{\circ} \mathrm{C}, \text { ramping to } 120^{\circ} \mathrm{C} \text { for } 60 \\
\text { min }\end{array}$ \\
\hline 4 & Etching & $\begin{array}{l}\text { March IRE Jupiter II, } 90 \% / 10 \% \quad 270 \mathrm{sccm} \\
\mathrm{O}_{2} / 28 \mathrm{sccm} \mathrm{CF}_{4}, 200 \text { Watts for } 20 \text { seconds }\end{array}$ \\
\hline
\end{tabular}

by method of mechanical lift off, a process often used in creation of MEMS devices. This process may be interesting to use, especially in a commercial process, because of its ease in integration into todays semiconductor processing; especially the use of photo-lithography as a patterning step, since it provides a straightforward path for large integration scaling where really fine features are required (eg. a pixel display).

As previously mentioned, wet processing is often ignored for polymers as chemical deterioration of the films is of major concern. Positive photoresist (PR) does not mix well with PEDOT:PSS which is highly acidic, and causes uncontrolled decomposition of the PR, while negative PR processing involves cross-linking with UV radiation which leaves residual material on the surface, compromising the PEDOT:PSS film. However, Lang et. al (2007) [37] and Ouyang et al. (2014) [36] have demonstrated that through careful selection of materials for deposition and processing, the PEDOT:PSS films can be minimally damaged during liftoff process.

For this process, negative resist SU8-2005 (MicroChem) was used, since it provides the most compatible processing properties (chemical, thermal) with PEDOT:PSS. SU8-2005 was diluted with a SU8 thinner such that the layers spun would be around $200 \mathrm{~nm}$, making them easier to lift off. All the same Si wafer conditioning steps as in section 4.2 were followed. The SU8 dilution was dispensed on a static wafer, spun at 1000 RPM form 30 seconds to wet the surface, then spun at 4000 RPM for 30 seconds to reach the a thickness of $100 \mathrm{~nm}$. The wafer is then placed on a hot plate at $95^{\circ} \mathrm{C}$ for a soft bake for 1 minute. For the purpose of exposure, an emulsion mask was created by the microfab technician, seen in Figure 4.5. Once the film has been soft baked, it was exposed on a Karl Suss MA 6 lithography machine under $80 \mathrm{~mJ} / \mathrm{cm}^{2}$ for 1 minute. For a negative tone resist, the exposed areas represented the areas in which 
the resist would stay after development, meaning the areas that were not the active device. After exposure another soft bake at $95^{\circ} \mathrm{C}$ for 1 minute is needed, and then the wafer was dipped in SU8 Developer (PGMEA based developer) for 1-2 minutes (or lift off of the active areas were seen) and then dipped in an IPA bath and dried by blowing a $N_{2}$ gas gun on it. The wafer then has an area of exposed $\mathrm{SiO}_{2}$ where the PEDOT:PSS will remain, while having the exposed areas with hardened crosslinked SU8 to be lifted off. To spin on the PEDOT:PSS layer, the same procedure for Baytron $\mathrm{P}$ as in section 4.2 were followed, except for the initial descum/Ozone plasma conditioning, as this could remove the SU8 layer. However, a wafer conditioning was done earlier in the process, which changed the surface energy of the wafer and helped with the Baytron $\mathrm{P}$ adhesion. Once the layer was spun on, the PEDOT:PSS layer now had to be baked, but it was done at a much lower temperature $\left(80^{\circ} \mathrm{C}\right)$ for 25 minutes, as putting it on a hot plate with the original time and temperature would constitute as a "hard bake" for the SU8 layer, making it virtually impossible to lift off.

For the lift off procedure of already cured SU8, MicroChem's documents indicate to use a heated up SU8 Developer and an ultrasonic bath. Following these instructions, the SU8 developer was heated to $80^{\circ} \mathrm{C}$, and the beaker was then placed in an ultrasonic bath, the wafer was submersed in the beaker and left to sonicate and develop for 180 minutes. Afterwards, the wafer was dipped in IPA and blown dry. The edges of the PEDOT:PSS were well defined, even in the smaller features. However, conductivity seemed to have decreased quite a bit which was hypothesized to have happened because of the long time it was submerged in the SU8 Developer. Digital Multimeter Resistance measurements for devices of 3 squares were averaging $4 \mathrm{M} \Omega$ as compared to around the $1 \mathrm{M} \Omega$ found from plasma etching.

An alternative to the above problem could be solved by the addition of an extra layer, in this case using MicroChem's OmniCoat. This layer has a higher reactivity to a PGMEA solvent, and as such will lift off faster than the SU8. To process this, all the same Si wafer conditioning steps as in section 4.2 were followed. OmniCoat dispensed on a static wafer, spun at 1000 RPM form 30 seconds to wet the surface, then spun at 4000 RPM for 30 seconds, yielding a $80 \mathrm{~nm}$ layer. A soft bake at $95^{\circ} \mathrm{C}$ for 1 minute was performed, and then the same exact procedure for the SU8 deposition, patterning and removal was performed. With this stack of materials, it was found that the ultarsonic bath removal step only had to be done for 90 minutes. 


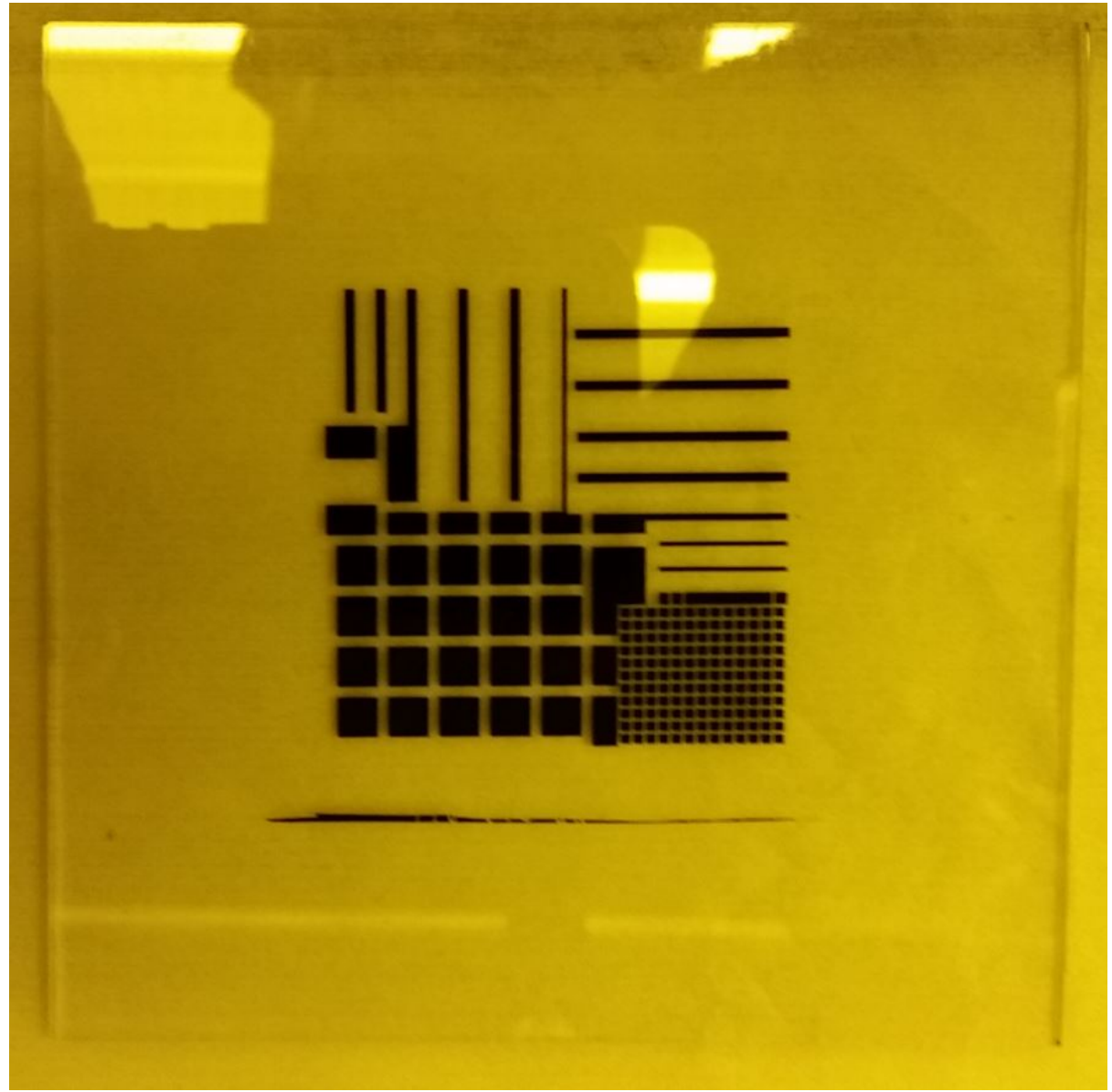

Figure 4.5: Emulsion mask for lithographic etching of PR

The conductivity seemed to have suffered less, as resistances of around $3 \mathrm{M} \Omega$ were measured.

\subsection{Metal Deposition}

To be able to properly contact the PEDOT:PSS for probing, metal contacts had to be deposited. Originally Aluminium (Al) was to be used, but through experimental procedure it was discovered that due to the acidic nature of PEDOT:PSS, at the $\mathrm{Al}$ and PEDOT:PSS interface the Al would oxidise into $\mathrm{AlO}_{x}$ [12] which would act as an insulator and the PEDOT:PSS layer could not be probed. With this precaution in mind, Silver (Ag) was used as the material for the pads.

For this application, regular physical metal evaporation techniques would not really work, since they require the wafer to stay inside a chamber where the desired 
Table 4.5: PEDOT:PSS Lift-off process (without OmniCoat)

\begin{tabular}{|c|c|c|}
\hline Step & Description & Details \\
\hline 1 & Wafer Conditioning & $\mathrm{O}_{2}$ plasma, 5 minutes \\
\hline 2 & PR Deposition & $\begin{array}{l}\text { SU8 Dilution, spin coated at } 1000 \text { RPM for } 30 \mathrm{~s} \text {, } \\
4000 \mathrm{RPM} \text { for } 30 \mathrm{sec}\end{array}$ \\
\hline 3 & Soft Bake & Hot plate, $95^{\circ} \mathrm{C}$ for $1 \mathrm{~min}$ \\
\hline 4 & Exposure & Karl Suss MA 6, $80 \mathrm{~mJ} / \mathrm{cm}^{2}$ for 1 minute \\
\hline 5 & Soft Bake & Hot plate, $95^{\circ} \mathrm{C}$ for $1 \mathrm{~min}$ \\
\hline 6 & Development & $\begin{array}{l}\text { SU8-Developer (PGMEA), 1-2 minutes, light agi- } \\
\text { tation }\end{array}$ \\
\hline 7 & Rinse & IPA Rinse, $10 \mathrm{sec}$, light agitation \\
\hline 8 & PEDOT:PSS Deposition & $\begin{array}{l}\text { PEDOT:PSS/IPA Dilution, spin coated at } 500 \\
\text { RPM for } 15 \mathrm{sec}, 1000 \mathrm{rpm} \text { for } 1 \text { minute }\end{array}$ \\
\hline 9 & PEDOT:PSS Hard Bake & Hot pate, $80^{\circ} \mathrm{C}$ for 25 minutes \\
\hline 10 & SU8 Lift-off & $\begin{array}{l}\text { SU8-Developer (PGMEA) at } 80^{\circ} \mathrm{C} \text {, ultrasonic bath } \\
\text { for } 180 \text { minutes }\end{array}$ \\
\hline
\end{tabular}

metal is melted at a much higher temperature than the PEDOT:PSS can withstand without thermal decomposition. To avoid this, a thermal evaporation Angstrom Engineering Covap system was used, which is an evaporation system that operates at much lower temperatures than regular evaporators (around $40^{\circ} \mathrm{C}$ instead of upwards of $400^{\circ} \mathrm{C}$ ) which makes it appropriate for usage in Organic device processing.

The wafer was placed in a metal chuck, and then the shadow mask was aligned and placed over it, then held down with magnets (Figure 4.6). The chuck was then placed inside of the Covap system, and the crucible was loaded up with 99.9\% pure Ag pellets. The machine has pre-programmed "recipes" which select the rate of deposition and thickness of the film, which was set to $5 \AA$ /sec and $1000 \AA$ respectively. The vacuum pump was activated and the chamber pressure was let to go down to about $8 \times 10^{-7}$ Torr, at which point the machine deposition was started. However, it was not found until after the device completion that the tooling factor for the machine had been calibrated incorrectly. The actual thickness of the contacts was measured to be around $210 \mathrm{~nm}$. This thicknes was deemed acceptable for metal contacts. 


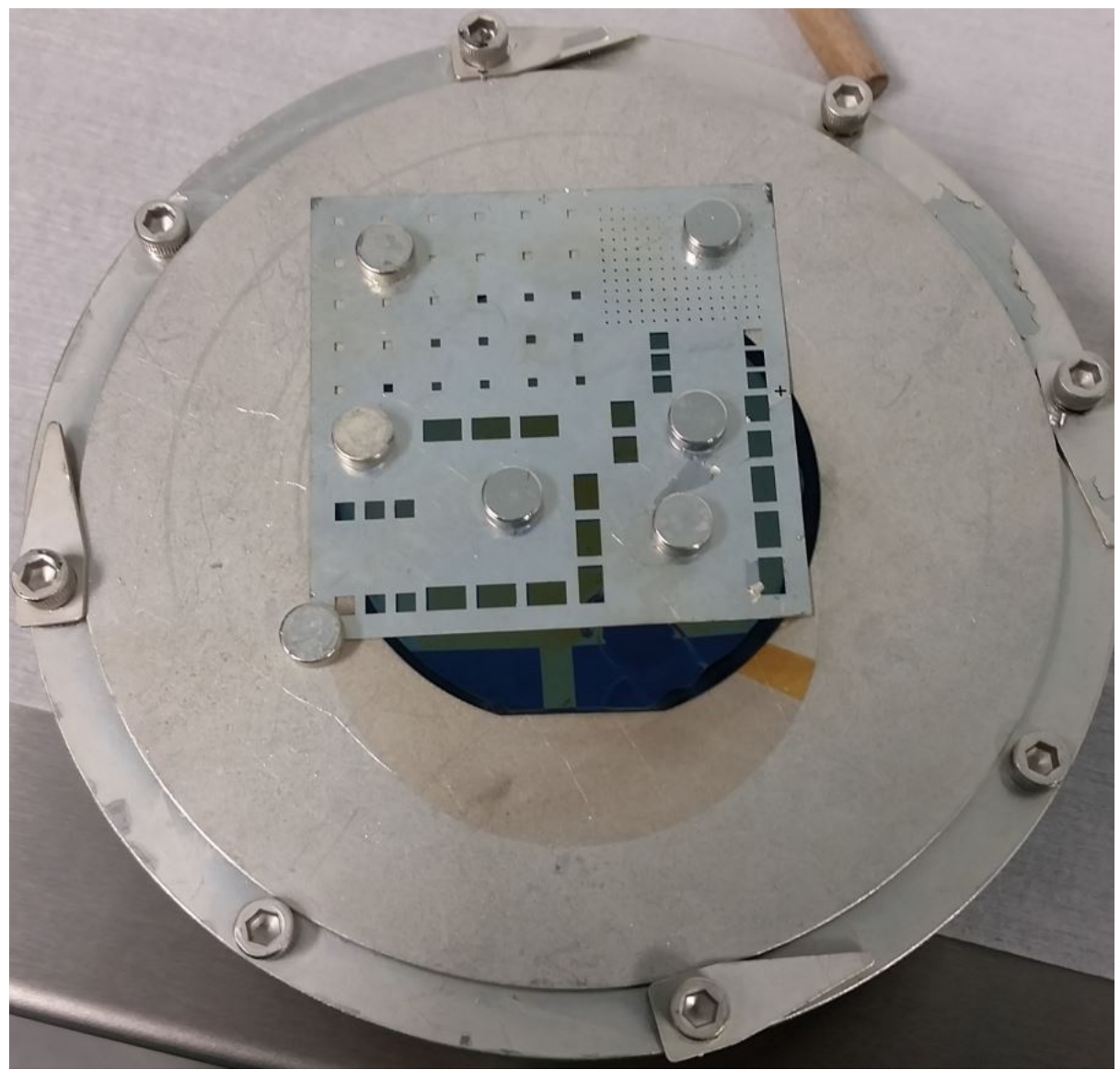

Figure 4.6: Wafer with mask ready for metallization

\section{6 bR Deposition}

The final step of the device production was to disperse a bR concentrate over the PEDOT:PSS active area. Commercial grade Wildtype bR in lyophilized form was obtained. The $1 \mathrm{mg}$ of bR was diluted with DI Water ( $\mathrm{pH} 6.8$ ) to obtain a concentration of $10 \mathrm{mg} / \mathrm{mL}$. Using a Micro pipette, about $15-25 \mu \mathrm{m}$ of the bR solution were dispersed across 8 different PEDOT:PSS covered areas, creating 7 different devices (of 4 different total sizes).

To dry the droplets, a procedure called Electric Field Sedimentation (EFS) was used. This procedure uses the net charge of the membrane to its advantage: it has been generally accepted that bR has its $\mathrm{CP}$ side more negatively charged than its EC side [38]. This net electric dipole moment can be then used to manipulate the orientation of bR film to point towards the anionic component of the PEDOT:PSS film, such that the EP side (the output of the ion pump) will be directed towards 
the conducting polymer film, increasing the photo-efficiency of the protein and maximizing the chance of de-doping of the PEDOT. For this procedure, the wafer was placed between 2 metal plates $5 \mathrm{~mm}$ apart, which will act as electrodes (See Figure Below). A DC Source was connected to each plate, with the bottom plate acting as ground. The top plate was placed at a bias of $10 \mathrm{~V}$ (yielding an electric field density of $20 \mathrm{Vcm}^{-1}$ ) and the droplets of bR were left to dry overnight for 12 hours. This whole poling assembly was held inside of a glove box held at $25^{\circ} \mathrm{C}$ and $48 \%$ humidity. Figure 4.7 shows a schematic of the setup used for the process.

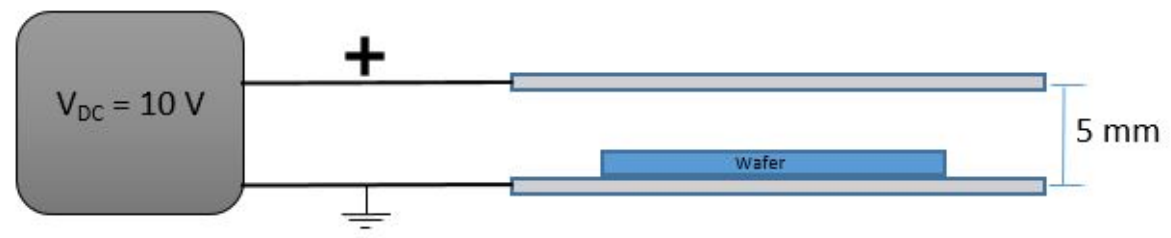

Figure 4.7: bR/PEDOT:PSS complete device

Figure 4.8 below shows a finalized bR/PEDOT:PSS device on silicon with metal contacts that will be tested in the next chapter.

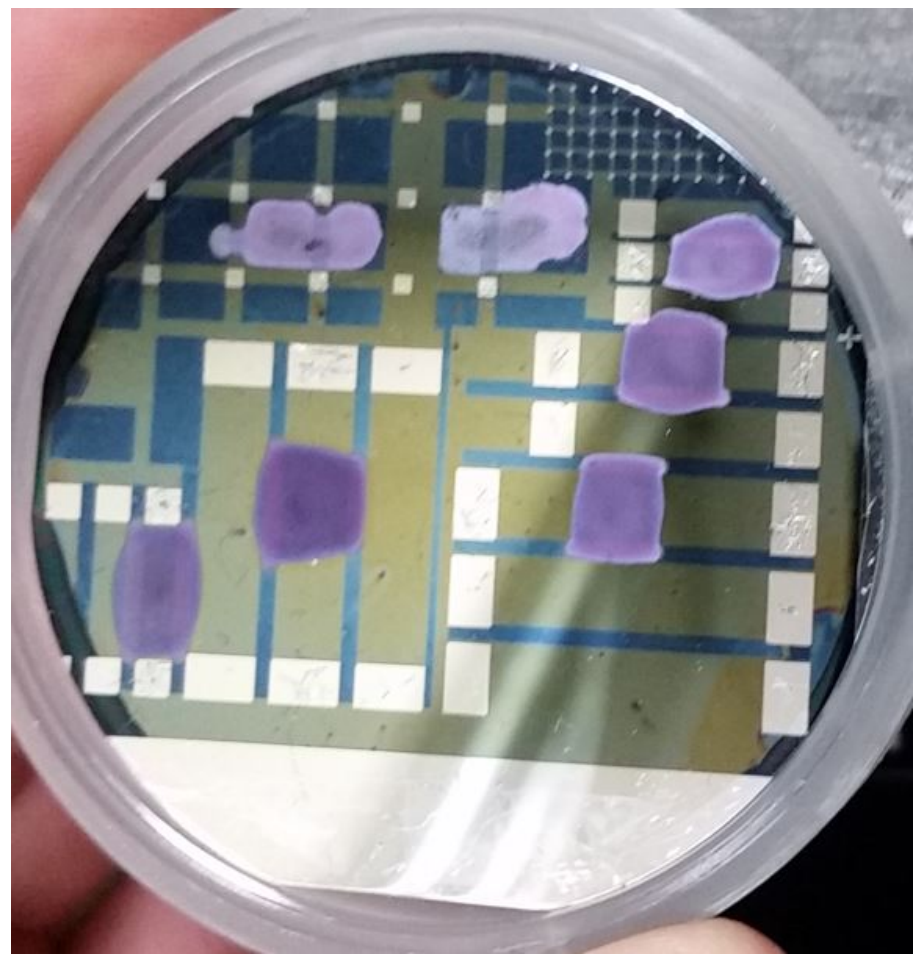

Figure 4.8: bR/PEDOT:PSS complete device 


\section{Chapter 5}

\section{Results and Discussion}

Various measurements were performed on the bR/PEDOT:PSS lateral device fabricated according to Chapter 4 . The testing involves both direct and indirect measurements of changes in resistance of the PEDOT:PSS film when responding to a probing laser. This chapter will outline the testing methodologies and set-ups, as well as discuss the results.

For these tests, 7 different devices with 4 different sizes were tested; below in Table 5.1 shows the devices tested, their size and thickness (image of the device can be seen in Figure 4.8)

Table 5.1: Tested Devices

\begin{tabular}{||ccc||}
\hline Device Name & Device Size $(\mu \mathrm{m})$ & Thickness $(\AA)$ \\
\hline \hline A1 & $3000 \times 12000$ & 986.63 \\
\hline A2 & $3000 \times 12000$ & 956.55 \\
\hline B1 & $4000 \times 16000$ & 1046.04 \\
\hline B2 & $4000 \times 16000$ & 1163.70 \\
\hline C1 & $2000 \times 8000$ & 907.82 \\
\hline D1 & $1000 \times 4000$ & 935.15 \\
\hline D2 & $1000 \times 4000$ & 919.32 \\
\hline
\end{tabular}




\subsection{Mobility Measurements}

The first important measurement to find out about the PEDOT:PSS layer is its conductivity, and finding out what the carrier mobility in the device is. This will give an insight into the conduction of the material, as well as be useful to benchmark against other semiconductor devices.

The sheet resistance of the PEDOT:PSS film was calculated using a four point probe method. Here, four terminals of a PEDOT:PSS strip are probed by equally spaced probes; the two outer source current while the middle two probes measure voltage. The difference in voltage between the middle probes is then plotted as a function of supplied current, and the slope of the graph is used with the empirically derived equation [39]

$$
\rho_{\square}=\frac{V}{I} \frac{\pi}{\ln 2}
$$

Or bulk resistivity can be calculated by incorporating the thickness of the film being probed:

$$
\rho=t \frac{V}{I} \frac{\pi}{\ln 2}
$$

Figure 5.1 below shows a 4 point probe measurement made on two PEDOT:PSS wafers, one that has been just fabricated, and one that has remained in a room at regular temperature and humidity for over a month.

Both devices show a similar I-V characteristic curve, but at higher currents the voltage seems to saturate. When conducting a 4 point probe measurement, negative charges are being pumped into the PEDOT:PSS film and the voltage across it is being monitored. The PEDOT:PSS film which is in a doped oxidized state will be reduced by these negative charges, effectively converting it into an insulator. It is possible for low conductivity formulations, such as Baytron $\mathrm{P}$, that once a certain current density is reached the PEDOT:PSS layer is completely oxidized and becomes an insulator, which Figure 5.1 seems to confirm.

The calculated sheet resistances for each device are:

$$
\rho_{\square, \text { Old }}=543.37 \frac{k \Omega}{\square}
$$


I-V curve for PEDOT:PSS

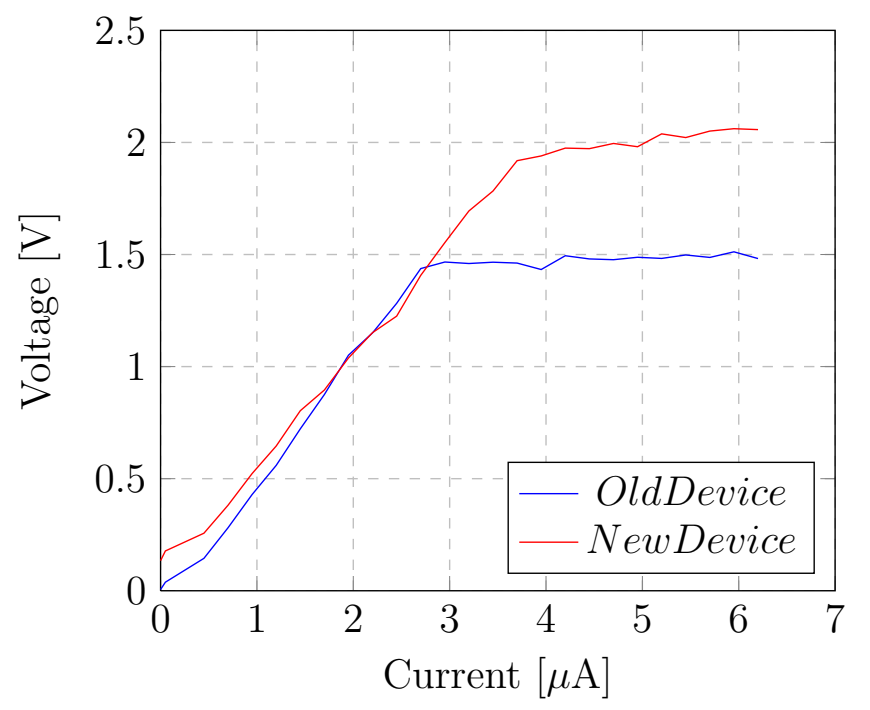

Figure 5.1: Current dependant Voltage of two PEDOT:PSS devices

$$
\rho_{\square, N e w}=491.87 \frac{k \Omega}{\square}
$$

Note that both devices show approximately the same sheet resistance as expected, however both devices can show a large difference in resistance as seen in Table 5.2 below:

Table 5.2: Thickness and Resistance of New vs Old PEDOT:PSS spin device

\begin{tabular}{||ccccc||}
\hline \multirow{2}{*}{ Device Name } & \multicolumn{2}{c}{ Old Device } & \multicolumn{2}{c}{ New Device } \\
& $\begin{array}{c}\text { Resistance } \\
(\mathrm{M} \Omega)\end{array}$ & $\begin{array}{c}\text { Original } \\
\text { Thickness }(\AA)\end{array}$ & $\begin{array}{c}\text { Resistance } \\
(\mathrm{M} \Omega)\end{array}$ & $\begin{array}{c}\text { Original } \\
\text { Thickness }(\AA)\end{array}$ \\
\hline \hline $\mathrm{A} 1$ & 1.836 & 986.63 & 1.275 & 922.9 \\
\hline $\mathrm{A} 2$ & 1.732 & 956.55 & 1.082 & 1024.71 \\
\hline $\mathrm{B} 1$ & 0.971 & 1046.04 & 0.845 & 1135.78 \\
\hline $\mathrm{B} 2$ & 1.082 & 1163.70 & 0.842 & 922.29 \\
\hline $\mathrm{C} 1$ & 2.78 & 907.82 & 1.29 & 934.08 \\
\hline $\mathrm{D} 1$ & 1.86 & 935.15 & 1.195 & 975.14 \\
\hline $\mathrm{D} 2$ & 1.36 & 919.32 & 1.229 & 908.14 \\
\hline
\end{tabular}

The films show to lose conductivity over time (some quite significantly), most likely 
due to the swelling of the devices by water uptake. Elschner et al [12] showed that PEDOT:PSS is strongly hygroscopic and when exposed to moisture in the air, thin films can experience an increase of up to 15 weight percentage. This tends to increase the thickness of the device and according to Equation 5.2, would therefore increase the bulk resistivity. The uptake of moisture also introduces protons to the film which will de-dope the PEDOT:PSS and increase resistivity. Elschner also showed that long time exposure to UV light degenerate PEDOT:PSS, as the UV can break the polymer bonds. For any sort of commercial applications, it is of great importance to encapsulate the device to avoid rapid degradation over the usable lifetime.

Having measured the thickness and calculated the sheet resistivity of the PEDOT:PSS, the bulk resistivity can be calculated, as well as the conductivity (Figure $5.3)$ :

Table 5.3: Resistivity and Conductivity of a PEDOT:PSS film

\begin{tabular}{||ccc||}
\hline Device Name & Resistivity $\rho(\Omega$-cm $)$ & Conductivity $\sigma(\mathrm{S} / \mathrm{cm})$ \\
\hline \hline A1 & 5.36 & 0.19 \\
\hline A2 & 5.20 & 0.19 \\
\hline B1 & 5.68 & 0.18 \\
\hline B2 & 6.32 & 0.16 \\
\hline C1 & 4.93 & 0.20 \\
\hline D1 & 5.08 & 0.20 \\
\hline D2 & 5.00 & 0.20 \\
\hline
\end{tabular}

The average conductivity was measured to be:

$$
\sigma_{\text {avg }}=0.19 S / \mathrm{cm}
$$

Although Bayton $\mathrm{P}$ is advertised as a $1 \mathrm{~S} / \mathrm{cm}$ conducting polymer, results show that the conductivity is actually lower. The mixture of conducting polymer used was very old, which may lead to possible aggregation of the polymer chains, causing tangles and lowering the conductivity, or even possibly just the breakdown of the polymeric chains. However, this is not a problem in the scope of this thesis, as high conductivity is not really required.

With the conductivity of the film obtained, a calculation of the theoretical change 
in conductivity can be approximated by trying to estimate the amount of protons which will be pumped into the PEDOT:PSS. This can be done since PEDOT:PSS is a semiconductor material, to which the mobility equation can be applied [10]

$$
\sigma=q \mu_{p} p+q \mu_{n} n
$$

But as a majority hole carrier device, the equation can be simplified:

$$
\sigma=q \mu_{p} p
$$

However, finding a mobility number $\left(\mu_{p}\right)$ in PEDOT:PSS is not that easy, since the conduction mechanism is not well understood, and empirical observations have not been able to determine a concrete number.

To approximate the number of holes, first the number of photons which will hit the bR sample must be calculated. This is dependant on both the energy of the photons and the intensity of the incident laser.

The energy of the photon can be calculated as [40]

$$
E_{\lambda}=\frac{h c}{\lambda}
$$

where $\mathrm{h}$ is Planck's constant $[\mathrm{J}-\mathrm{s}]$ and $\mathrm{c}$ is the speed of light $\left[\mathrm{m} / \mathrm{s}^{2}\right]$. Once the photon energy has been found, dividing the light intensity $\mathrm{I}\left[\mathrm{W} / \mathrm{m}^{2}\right]$ yields a rate of photons in an area, $N_{p}$ :

$$
N_{p}=\frac{I}{E_{\lambda}}
$$

The laser can be approximated as a $1.5 \times 1.5 \mathrm{~mm}$ square area of irradiation (for simplicity of calculations), so dividing this area by the intensity will give the flux of photons in the designated area. Figure 5.2 is a graph that shows the correlation between light intensity and the number of photons crossing through a $1.5 \times 1.5 \mathrm{~mm}$ area.

In the next section, the actual resistance change caused by irradiation with a green laser has been measured and a model accounting for the change in resistance has been proposed. The equations from this section will be used to determine the change in conductivity caused by the irradiation and the number of holes required for this change, which in turn can be used to find the amount of photons absorbed 


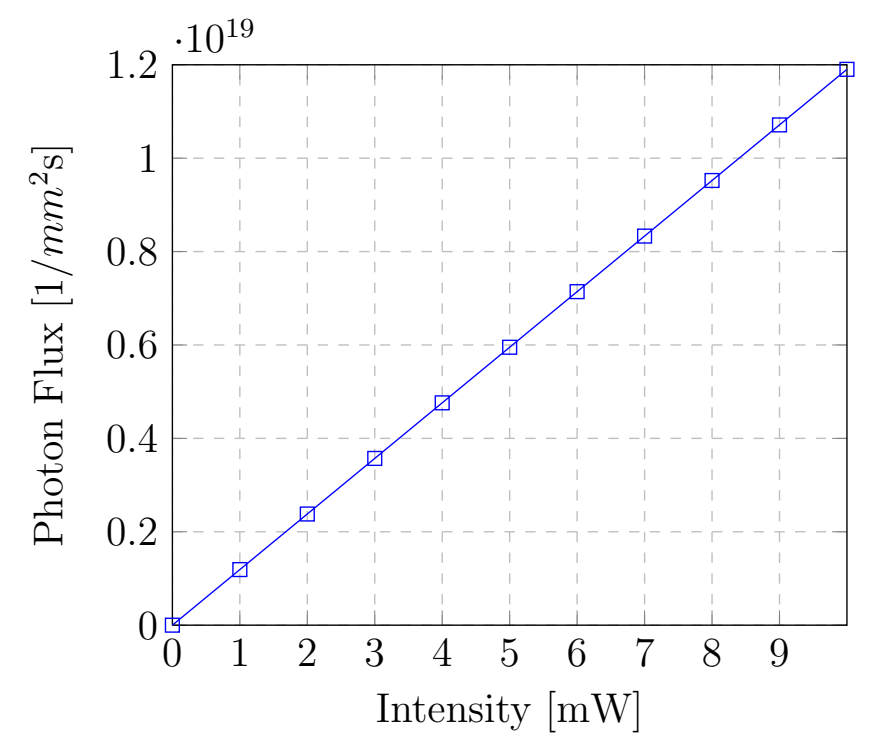

Figure 5.2: Number of Photons irradiated by a $532 \mathrm{~nm}$ Green Laser in a $1.5 \mathrm{x} 1.5 \mathrm{~mm}$ area

to compare to the theoretical efficiency.

\subsection{Photoresponse Measurements}

One of the first important measurements to confirm the operation of the device is to observe if there is any change in the resistance when hit by an incident probing laser. By doing a DC measurement of the change in resistance, two things can be confirmed: the bR layer is pumping protons and de-doping the PEDOT:PSS and that the drying method of EFS does indeed help the orientation of the bR molecules.

For this test set-up, probes were connected to the Ag pads of different devices, which were then connected to a Keithley 177 Microvolt DMM. Once the bR patch is shun with light, the "light turn-on" change in resistance could be directly read from the DMM, which would then stabilize to the original resistance value as the charges transferred over from the initial dipole moment switch in bR come to equilibrium with the chemical gradient created by the proton pumping [1]. Two wavelengths of light would be used for this test: one close to the peak absorption (Green 532 $\mathrm{nm}$ laser) and one at a lower absorption (Red $632 \mathrm{~nm}$ laser). Having two different wavelengths should yield different absorptions of photons, meaning that different amount of protons would be pumped and the resistance change would be different for each wavelength. As a control for this test, a strip of PEDOT:PSS without bR was 
illuminated with both wavelength lasers to see if they induced a change in resistance, which they did not. For consistency, both lasers were set to the same power $(6.3 \mathrm{~mW})$ and to illuminate the same spot in the bR patch, which yielded the following results:

Table 5.4: Tested Devices

\begin{tabular}{||cccc||}
\hline Device Name & $\begin{array}{l}\text { No Light Resistance } \\
(\mathrm{k} \Omega)\end{array}$ & $\begin{array}{l}632 \mathrm{~nm} \text { Red Laser } \\
\text { Resistance }(\mathrm{k} \Omega)\end{array}$ & $\begin{array}{l}532 \mathrm{~nm} \text { Green Laser } \\
\text { Resistance }(\mathrm{k} \Omega)\end{array}$ \\
\hline \hline A1 & 2713.24 & 2785.11 & 2835.89 \\
\hline B1 & 1609.45 & 1625.31 & 1638.08 \\
\hline B2 & 1334.77 & 1348.12 & 1359.54 \\
\hline A2 & 3422.33 & 3456.24 & 3487.21 \\
\hline C1 & 2206.78 & 2200.01 & 2204.76 \\
\hline D1 & 2215.12 & 2466.75 & 2571.33 \\
\hline D2 & 2066.11 & 2197.45 & 2315.27 \\
\hline
\end{tabular}

As predicted, shining a the bR patch with a green laser (approx. 65\% absorption) causes a larger de-doping of the PEDOT:PSS layer (higher resistance) than the red laser (approx. 30\% absorption), which corresponds to a larger probability of absorbing the light quanta which would induce a proton to be pumped. Also to note is the increased resistance between these values and the ones in Table 5.2, as this test was performed at an even later date than the ones in Table 5.2

This change in resistance can be used to approximate the amount of holes absorbed by the bR and compare it to the theoretical values proposed in Figure 5.2. First, the average resistance change of the devices was found as:

$$
\Delta R_{a v g}=120.57 k \Omega
$$

Which can then be used to find the conductivity change associated with this resistance change:

$$
\begin{aligned}
\Delta R & =\frac{\Delta \rho L}{A} \\
\Delta R & =\frac{L}{\Delta \sigma A}
\end{aligned}
$$




$$
\Delta \sigma=\frac{L}{\Delta R A}
$$

The consideration of the irradiation area being a $1.5 \times 1.5 \mathrm{~mm}$ square simplifies the equation to:

$$
\Delta \sigma=\frac{1}{\Delta R t_{\text {avg }}}
$$

Where $t_{\text {avg }}=987.89 \AA$ is the average thickness of the PEDOT:PSS layer. With this, the change in conductivity can be found as:

$$
\Delta \sigma=-0.084 \mathrm{~S} / \mathrm{cm}
$$

Here $\Delta \sigma$ is negative because the change in resistance is positive. Now, the new conductivity under irradiation can be found:

$$
\begin{gathered}
\Delta \sigma=\sigma_{2}-\sigma_{1} \\
\sigma_{2}=\Delta \sigma+\sigma_{1} \\
\sigma_{2}=(-0.084)+0.19 \\
\sigma_{2}=0.106 \mathrm{~S} / \mathrm{cm}
\end{gathered}
$$

Assuming that the carrier mobility $\mu_{p}$ stays constant, the ratio of conductivities between no light and illumination can be used to determine the actual amount of holes absorbed after illumination, or an estimation of the quantum efficiency of the PEDOT:PSS:

$$
\begin{gathered}
\frac{\sigma_{2}}{\sigma_{1}}=\frac{p_{2}}{p_{1}} \\
p_{1}=\frac{p_{2} \sigma_{1}}{\sigma_{2}}
\end{gathered}
$$

Using Figure 5.2, the theoretical number of photons being shun upon the area by a laser at $6.3 \mathrm{~mW}$ would be about $7.5 \times 10^{18}$ photons/sec, and assuming perfect conversion of photons to hole at peak absorption (65\%), the number of holes would be:

$$
p_{1}=4.875 x 10^{18} \text { holes }
$$




$$
\begin{gathered}
p_{2}=\frac{\left(4.875 \times 10^{18}\right)(0.106)}{(0.19)} \\
p_{2}=2.72 \times 10^{18} \text { holes }
\end{gathered}
$$

Which in turn would correspond to a photon number of:

$$
\begin{gathered}
N_{p}=\frac{2.72 \times 10^{18}}{0.65} \\
N_{p}=4.184 \times 10^{18} \text { photons }
\end{gathered}
$$

As it can be seen, the number of photons which were calculated to be absorbed (the ones that cause the change in conductivity) are actually much less than the ones irradiated, and even this number is an overestimation. This, however, does spark some insight into the quantum efficiency of the bR. It is unrealistic to expect all the light (even at peak absorption) to be absorbed by the bR, as it is quite likely that some of it is being lost to reflection and transmission, while some of the transmitted light may also be bouncing back off of the Si wafer and be absorbed.

Because of the assumption made about the irradiation area of the laser, it is important to develop a model for how this area affects the conduction path in the PEDOT:PSS film. The strip of PEDOT:PSS can be assumed as a network of resistors as shown in Figure 5.3, where the $1.5 \times 1.5 \mathrm{~mm}$ area will be a variable resistor $\left(R_{P D}\right.$, green area) which changes with the irradiated light. When the light width is smaller than the total width of the device, there will be parallel current paths (represented by fixed resistor $R_{2}$, blue area), which means that the total resistance in the middle section $\left(R_{\text {mid }}\right)$ will be the parallel combination of these resistors:

$$
R_{\text {mid }}=\frac{1}{\frac{1}{R_{2}}+\frac{1}{R_{2}}+\frac{1}{R_{P D}}}
$$

With the total resistance of the device as the series combination of the fixed side resistors $\left(R_{1}\right.$, orange area) and the middle area $\left(R_{\text {mid }}\right)$.

With the conductivity found in Table 5.3 and the change in conductivity found earlier in this section, an estimation of the percentage in resistance change attributed to the irradiated area can be estimated. Below is a sample calculation for the device A1, whose dimensions can be seen in Figure 5.4.

The fixed resistors $R_{1}$ and $R_{2}$ can be estimated using a conductivity of $0.19 \mathrm{~S} / \mathrm{cm}$ 


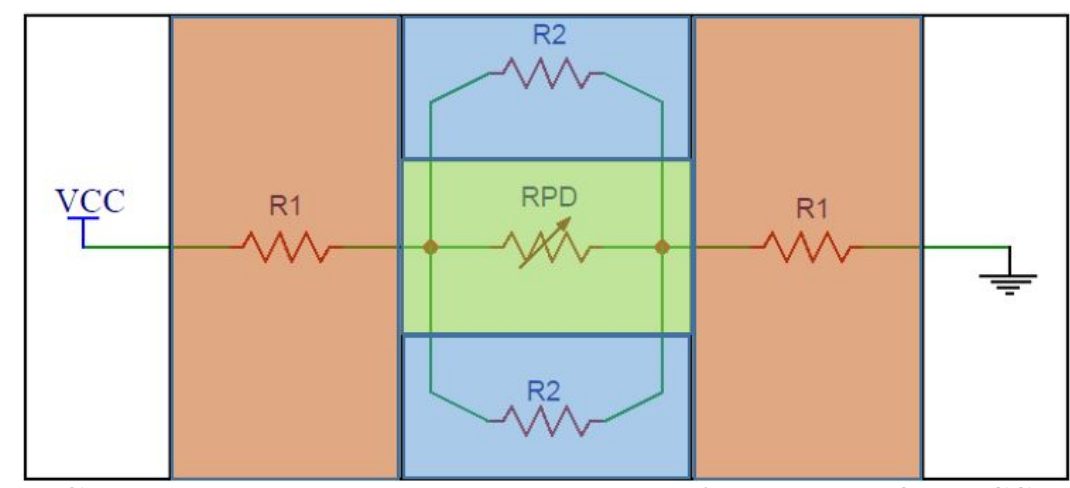

Figure 5.3: Circuit schematic model proposed for a PEDOT:PSS resistive strip where the Green area represents the illuminated bR/PEDOT:PSS interface.

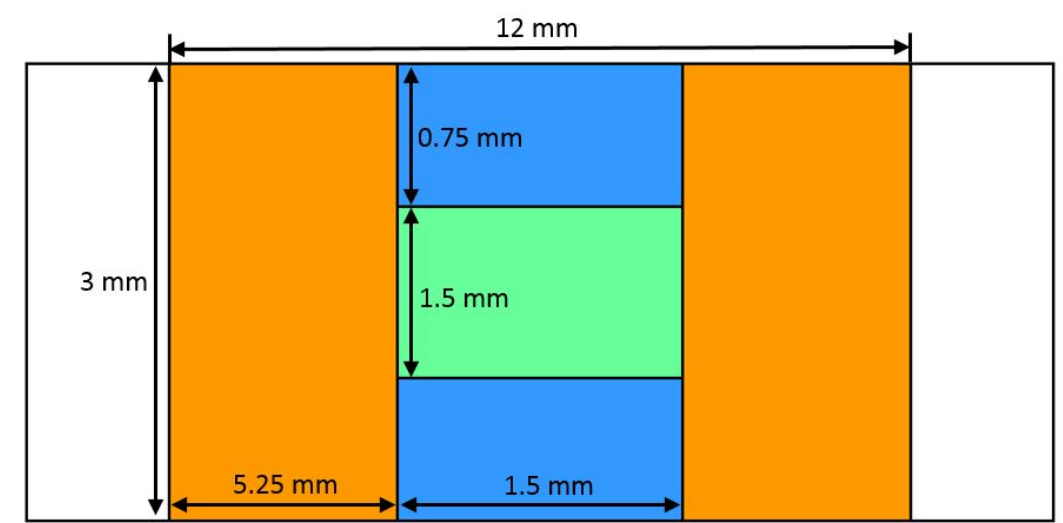

Figure 5.4: Dimensions for all the resistor sections in a bR/PEDOT:PSS device of size A1.

as per Table 5.3 and the dimensions found in Figure 5.4, as well as the thickness found in Table 5.2.

$$
\begin{gathered}
R_{1}=\frac{L}{\sigma A} \\
0.525 \mathrm{~cm} \\
R_{1}=\frac{R_{1}=95.07 \mathrm{k} \Omega}{(0.3 \mathrm{~cm})(0.19 S / \mathrm{cm})\left(9.8663 \times 10^{-6} \mathrm{~cm}\right)} \\
R_{2}=\frac{L}{\sigma A} \\
R_{2}=\frac{0.15 \mathrm{~cm}}{(0.075 \mathrm{~cm})(0.19 \mathrm{~S} / \mathrm{cm})\left(9.8663 \times 10^{-6} \mathrm{~cm}\right)} \\
R_{2}=108.65 \mathrm{k} \Omega
\end{gathered}
$$


The resistance of the illuminated $1.5 \times 1.5 \mathrm{~mm}$ area can then be calculated under two conditions: with no illumination (using the conductivity from Table 5.3, 0.19 $\mathrm{S} / \mathrm{cm}$ ) and under illumination (using the change in conductivity $\sigma_{2}$ found earlier in this section, $0.106 \mathrm{~S} / \mathrm{cm})$ :

$$
\begin{gathered}
R_{P D, \text { nolight }}=\frac{1}{(0.19 \mathrm{~S} / \mathrm{cm})\left(9.8663 \times 10^{-6} \mathrm{~cm}\right)} \\
R_{P D, \text { nolight }}=54.33 \mathrm{k} \Omega \\
R_{P D, \text { light }}=\frac{1}{(0.106 \mathrm{~S} / \mathrm{cm})\left(9.8663 \times 10^{-6} \mathrm{~cm}\right)} \\
R_{P D, \text { light }}=95.62 \mathrm{k} \Omega
\end{gathered}
$$

Now the parallel combination of the middle section of resistors $R_{m} i d$ can be calculated for both the light and no light conditions using Equation 5.7:

$$
\begin{gathered}
R_{\text {mid,nolight }}=27.64 k \Omega \\
R_{\text {mid,light }}=34.64 k \Omega
\end{gathered}
$$

So the total resistance of the device becomes:

$$
\begin{gathered}
R_{\text {nolight }}=2 R_{1}+R_{\text {mid, nolight }} \\
R_{\text {nolight }}=217.78 \mathrm{k} \Omega \\
R_{\text {light }}=2 R_{1}+R_{\text {mid,light }} \\
R_{\text {light }}=224.78 \mathrm{k} \Omega
\end{gathered}
$$

And the percentage of change can be calculated as:

$$
\begin{gathered}
\% \text { Change }=\frac{R_{\text {light }}-R_{\text {nolight }}}{R_{\text {nolight }}} x 100 \\
\% \text { Change }=3.21 \%
\end{gathered}
$$

Table 5.5 shows all the estimated resistances in each device, as well as the \% change in resistance that the illuminated area accounted for. One thing to note is that for devices of size D, the light covers the entire width of the device, meaning 
that there are no parallel current path $R_{2}$.

Table 5.5: Tested Devices

\begin{tabular}{||cccccccc||}
\hline Device Name & $R_{1}$ & $R_{2}$ & $R_{P D, \text { nolight }}$ & $R_{P D, \text { light }}$ & $R_{\text {Nolight }}$ & $R_{\text {Light }}$ & \% Change \\
\hline \hline $\mathrm{A} 1$ & $95.07 \mathrm{k} \Omega$ & $108.65 \mathrm{k} \Omega$ & $54.33 \mathrm{k} \Omega$ & $95.62 \mathrm{k} \Omega$ & $217.78 \mathrm{k} \Omega$ & $224.78 \mathrm{k} \Omega$ & $3.21 \%$ \\
\hline $\mathrm{A} 2$ & $95.13 \mathrm{k} \Omega$ & $108.72 \mathrm{k} \Omega$ & $54.36 \mathrm{k} \Omega$ & $95.91 \mathrm{k} \Omega$ & $217.45 \mathrm{k} \Omega$ & $224.96 \mathrm{k} \Omega$ & $3.34 \%$ \\
\hline $\mathrm{B} 1$ & $98.42 \mathrm{k} \Omega$ & $65.16 \mathrm{k} \Omega$ & $54.3 \mathrm{k} \Omega$ & $78.84 \mathrm{k} \Omega$ & $217.2 \mathrm{k} \Omega$ & $220.59 \mathrm{k} \Omega$ & $1.54 \%$ \\
\hline $\mathrm{B} 2$ & $98.44 \mathrm{k} \Omega$ & $65.17 \mathrm{k} \Omega$ & $54.31 \mathrm{k} \Omega$ & $95.62 \mathrm{k} \Omega$ & $217.24 \mathrm{k} \Omega$ & $219.93 \mathrm{k} \Omega$ & $1.22 \%$ \\
\hline $\mathrm{C} 1$ & $88.25 \mathrm{k} \Omega$ & $325.84 \mathrm{k} \Omega$ & $54.31 \mathrm{k} \Omega$ & $101.06 \mathrm{k} \Omega$ & $217.22 \mathrm{k} \Omega$ & $238.86 \mathrm{k} \Omega$ & $9.06 \%$ \\
\hline $\mathrm{D} 1$ & $43.46 \mathrm{k} \Omega$ & - & $81.48 \mathrm{k} \Omega$ & $147.16 \mathrm{k} \Omega$ & $168.40 \mathrm{k} \Omega$ & $234.07 \mathrm{k} \Omega$ & $28.06 \%$ \\
\hline $\mathrm{D} 2$ & $43.51 \mathrm{k} \Omega$ & - & $81.58 \mathrm{k} \Omega$ & $149.69 \mathrm{k} \Omega$ & $168.6 \mathrm{k} \Omega$ & $236.71 \mathrm{k} \Omega$ & $28.77 \%$ \\
\hline
\end{tabular}

As expected, devices where the illuminated area represents a smaller portion of the total area of the PEDOT:PSS resistive strip show a much smaller change in total resistance of the device, results which are in accordance to the ones found in Table 5.4, and to the ones found in the next section for the intensity dependant measurements. It should be noted that the numbers will not match up, because of the aforementioned environmental degradation of the PEDOT:PSS which accounts for much larger resistances than the ones theoretically derived in the calculations above.

Now that the device had been confirmed to work, another interesting property to characterize would be the dependence of the device on the incident light intensity. The 532 laser is connected to a DC power supply, and varying the input voltage would vary the intensity of the laser, so the 532 laser power (in watts) was measured with a laser intensity meter at different input voltages. Once the voltage points had been set, the bR/PEDOT:PSS devices were hooked up to the Keithley DMM, and the resistance at the turn on point were measured at different laser intensities. Figure 5.3 and 5.4 below show the intensities for devices of the same size plotted in the same graph.

As mentioned above, the incident light can be estimated as a $1.5 \times 1.5 \mathrm{~mm}$ square on the surface of the bR. As such, the percentage of area covered in each device will vary. This can be seen in the Figures 5.5 and 5.6, where smaller devices seem to show 
Intensity Dependant Resistance of bR

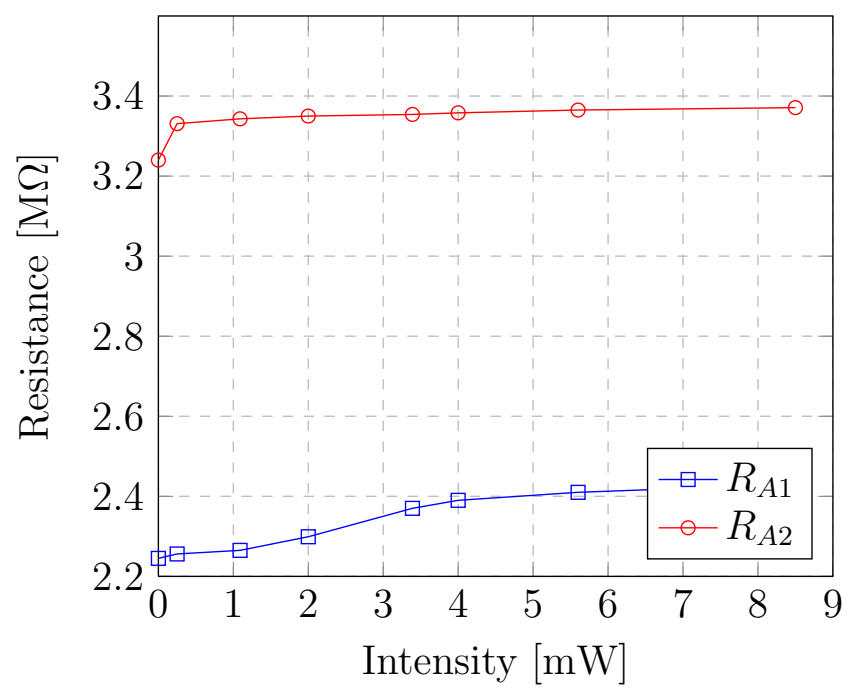

(a) Devices Size A

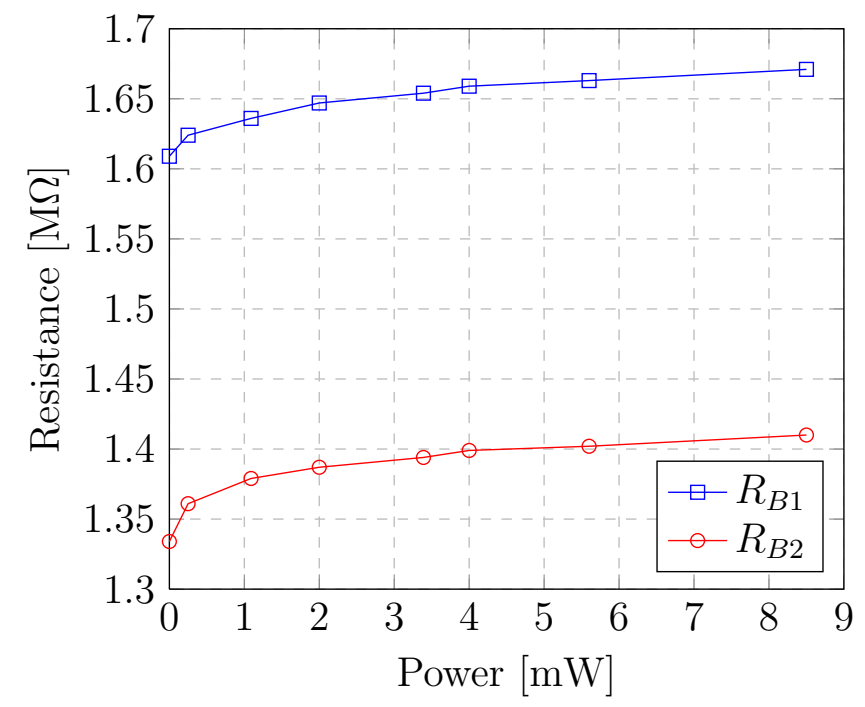

(b) Devices Size B

Figure 5.5: PEDOT:PSS film resistance dependant on incident $532 \mathrm{~nm}$ Laser Power for $\mathrm{A}$ and $\mathrm{B}$ sized devices 
Intensity Dependant Resistance of bR

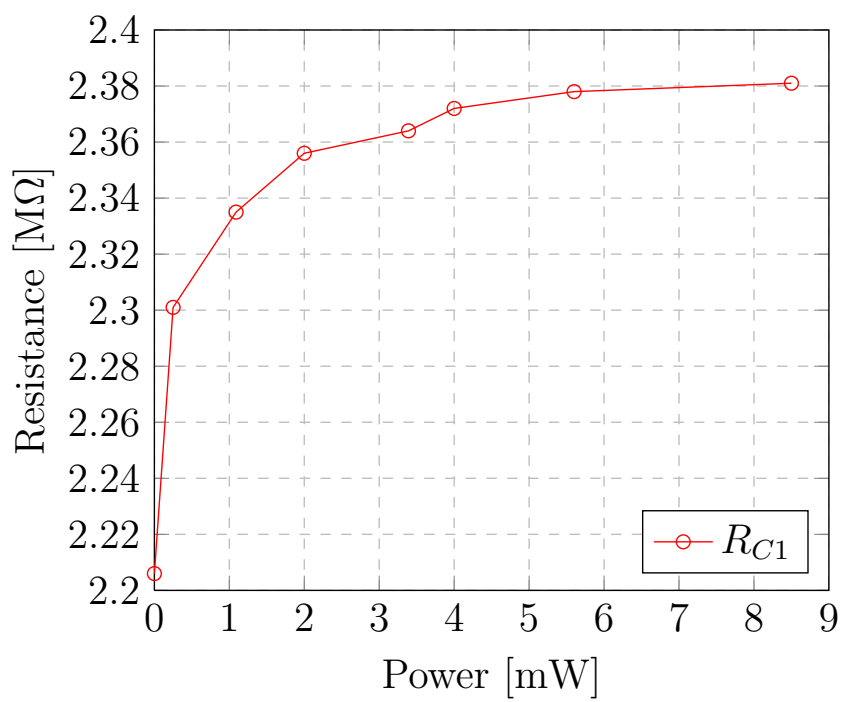

(a) Devices Size C

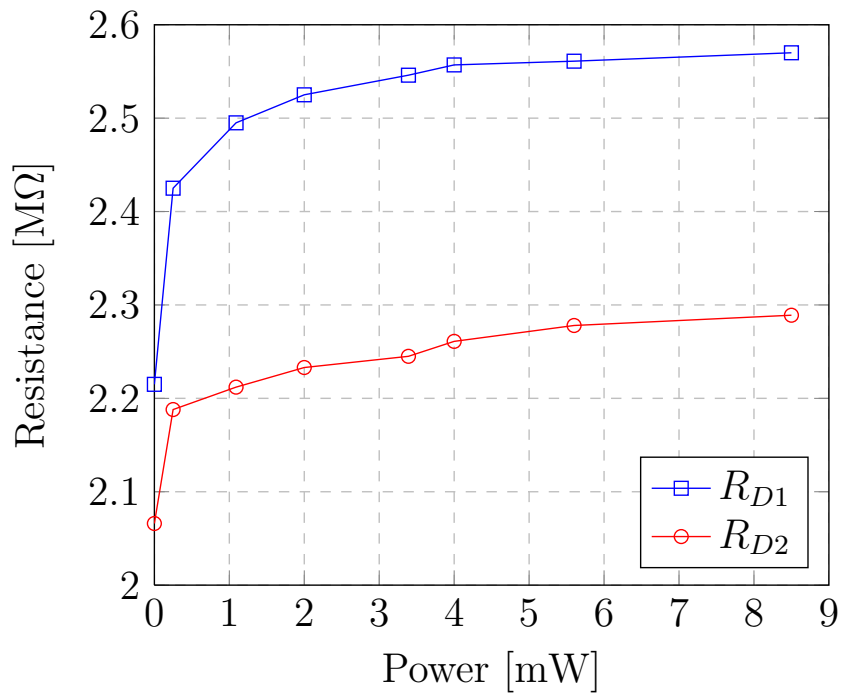

(b) Devices Size D

Figure 5.6: PEDOT:PSS film resistance dependant on incident $532 \mathrm{~nm}$ Laser Power for $\mathrm{C}$ and $\mathrm{D}$ sized devices 
a larger change in resistance in reaction to a photoexcitation. Figure 5.5 (a) and (b) show the response of the two largest devices, $3 \times 12 \mathrm{~mm}$ and $4 \times 16 \mathrm{~mm}$ respectively. The changes in resistance of both devices in Figure 5.5 (a) can be seen around 100$140 \mathrm{k} \Omega$, while the changes in Figure 5.5 (b) are approximately $50-60 \mathrm{k} \Omega$. For the smaller devices seen in Figure $5.6(2 \times 8 \mathrm{~mm}$ and $1 \mathrm{x} 4 \mathrm{~mm})$, the incident laser coverage represents almost the entirety (if not the entirety) of the width of the device, meaning that most of the PEDOT:PSS in the incident area will be de-doped, representing a much larger fraction of the area changing resistivity. This is noticeable in Figure 5.6 (a) where the change is about $170 \mathrm{k} \Omega$, and Figure 5.6 (b) where the change varies from $200-350 \mathrm{k} \Omega$.

The variation in the amount of resistance change could be attributed to the orientation of the bR molecules. As mentioned in Chapter 4, the bR molecules were left to dry under a $20 \mathrm{~V} / \mathrm{cm}$ electric field for 12 hours. However, it was not found until later that these were not ideal drying conditions. Min te al (1998) [41] conducted research on the optimal conditions for electrophoretic sedimentation drying for bR films by drying various bR films under different electric field densities and different exposure times, and checking the magnitude of the optoelectric response (in volts across 2 electrodes). The group found that the optimal drying electric field is $20 \mathrm{~V} / \mathrm{cm}$, and that exposure time of $45 \mathrm{sec}$ yielded the largest photoresponse, and that exposure times over 300 sec would damage the film, heavily hindering the photoresponse. In this work, the film was dried for 12 hours, much longer than the point of film damage, meaning that film orientation would most likely be compromised. Another issue with the EFS drying lies in the set up of the 2 conducting plates. While all possible efforts were taken, the top plate in the parallel plate structure could not be held perfectly horizontal, meaning that across the drying field there may have been electric field variations which could cause a difference in alignment of the bR molecules.

\subsection{Frequency Response}

An interesting feature to look at, especially for the potential application in the field of sensors, is how the bR/PEDOT:PSS device responds to irradiation at different frequencies. An analysis of the device's response to frequency modulation of the incident light can also give insight into the photocycle of bR, especially of the limiting transition step from $\mathrm{M}$ state to bR state which takes about $10 \mathrm{mSec}$. 
To conduct this measurement, the green laser was modulated by inputting a sine wave. The modulation input was connected to the output of a HP 33120A Function generator, and a $2.3 V_{p k-p k}$ sine wave was applied, corresponding to a peak-to-peak power of $2 \mathrm{~mW}$. Because the change in voltage would be hard to measure directly out of the device, since the change in voltage may be too small, an indirect measurement method had to be taken. For the measurement, the resistance of each individual device was measured with a DMM, and a voltage divider circuit with the same resistance as the PEDOT:PSS was constructed. The PEDOT:PSS will act as a variable photoresistor when irradiated by the green laser, changing the ratio of resistance and the voltage seen across the fixed resistor.

Because the actual output voltage would be very small, an amplifier with really high gain has to be used, as well as one that will extract the signal from an extremely noisy environment, since the noise and the desired signal are likely in the same order of magnitude. For this, a lock-in amplifier (LIA) was used, as it can amplify a carrier signal with a known frequency up to $120 \mathrm{~dB}$. The fixed resistor was connected to the input of a Itacho 3961 LIA, and the sync output of the function generator was connected to the sync input of the LIA, locking the modulated frequency of the laser and amplifier to the same frequency. Figure 5.7 shows a schematic of the testing set up.

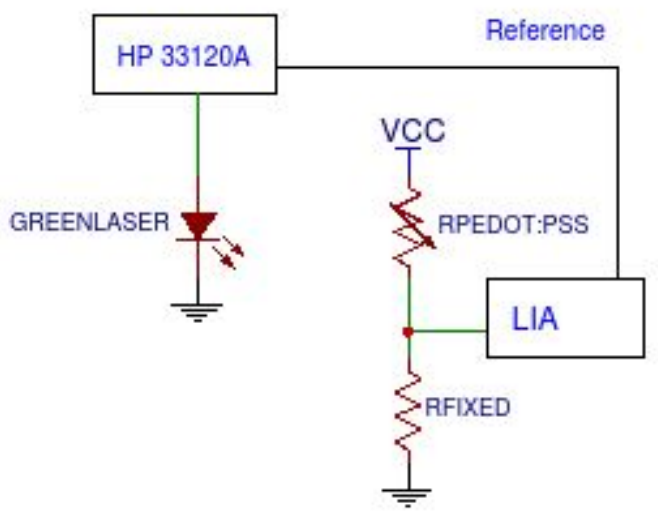

Figure 5.7: Testing setup for the measurement of bR/PEDOT:PSS frequency response

The equation for the voltage divider can be derived as: 


$$
V_{\text {out }}=V_{c c} \frac{R_{\text {fixed }}}{R_{\text {fixed }}+R_{P E D O T: P S S}}
$$

The values of the tested devices and fixed resistors are seen in Table 5.6

Table 5.6: Fixed resistors used for voltage divider

\begin{tabular}{||ccc||}
\hline Experiment & $R_{\text {PEDOT:PSS }}(\mathrm{M} \Omega)$ & $R_{\text {Fixed }}(\mathrm{M} \Omega)$ \\
\hline \hline $\mathrm{A} 1$ & 1.836 & 1.896 \\
\hline $\mathrm{B} 1$ & 0.971 & 0.964 \\
\hline $\mathrm{B} 2$ & 1.082 & 1.075 \\
\hline $\mathrm{A} 2$ & 2.78 & 2.78 \\
\hline $\mathrm{C} 1$ & 1.732 & 1.748 \\
\hline $\mathrm{D} 1$ & 1.86 & 1.853 \\
\hline $\mathrm{D} 2$ & 1.36 & 1.313 \\
\hline
\end{tabular}

For the measurement, the frequency would be swept from $10-10000 \mathrm{~Hz}$, and the output voltage is measured from the LIA. This measurement was repeated 3 times, and the average of these was plotted, with the multiple measurements giving some error margin to the data point. The "sync" output signal of the function generator could potentially inject some noise into the LIA, so it is also of interest to do dark (no laser) measurement of the output displayed by the LIA as a function of frequency. For this measurement, the sync output was connected to the LIA and the output of the function output was connected to the laser, but no DC voltage was given to the laser making it not illuminate. The fixed resistor was then connected to the input of the LIA and the frequency of the function generator was swept. One should not see any change in voltage, as there is no change in resistance going on with the device, meaning that any increase in output voltage would come from injected noise.

Like in figure 5.5 and 5.6, these will be presented for each individual device size and the dark measurement is averaged for multiple devices of the same size. Results are seen in figure 5.9 and 5.10, where the line indicates the average value of the measurements, the circle represents the maximum measurement and the star represents the lowest measured value. 


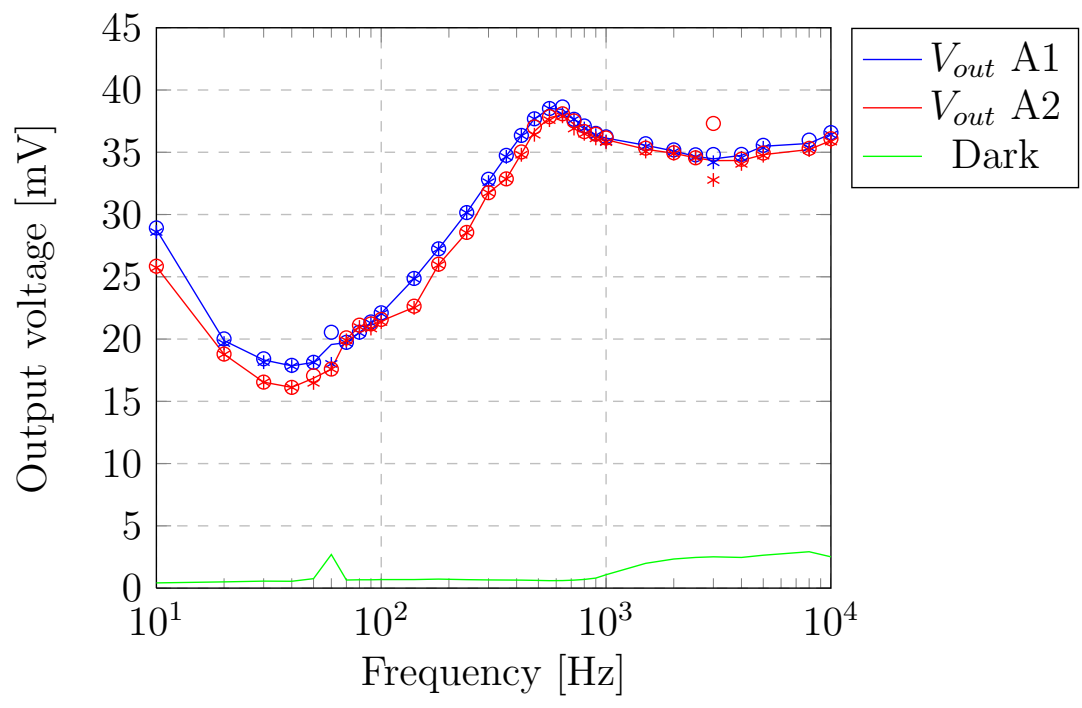

(a) Devices Size A

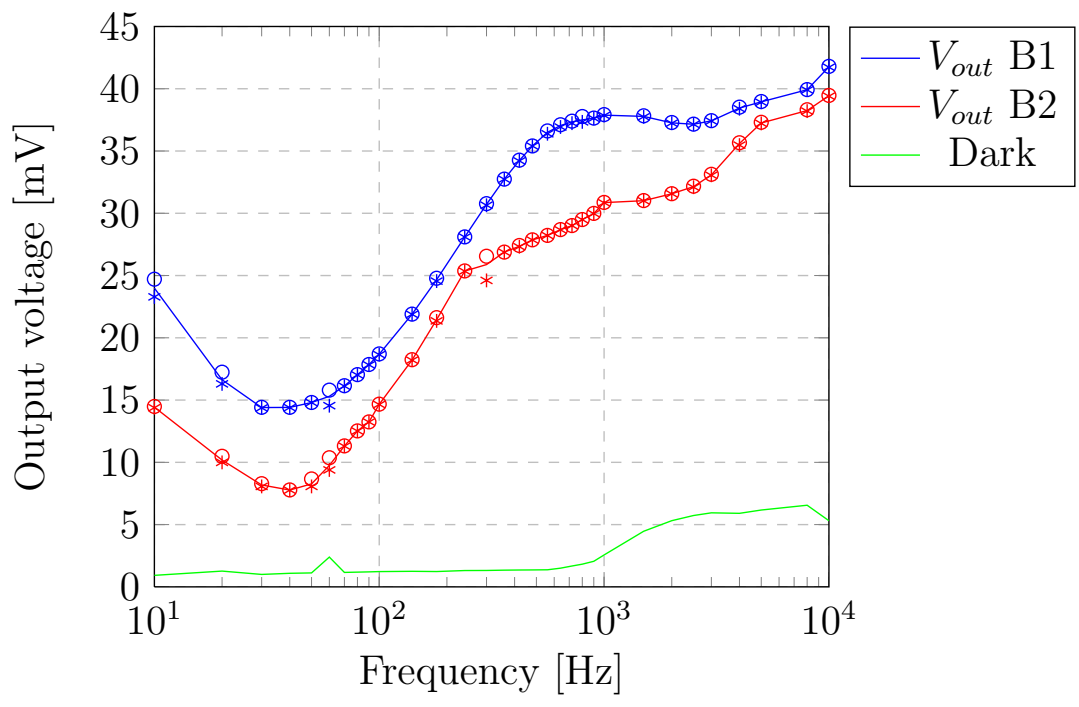

(b) Devices Size B

Figure 5.8: Frequency dependant response of bR/PEDOT:PSS devices of size A and $\mathrm{B}$ 


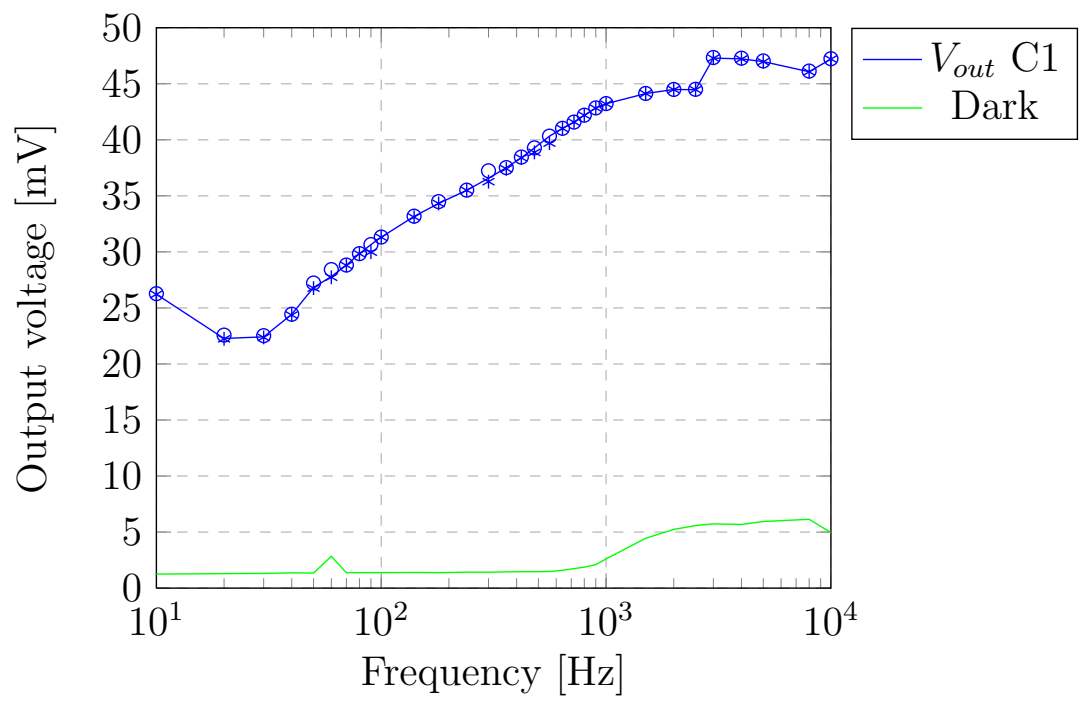

(a) Devices Size C

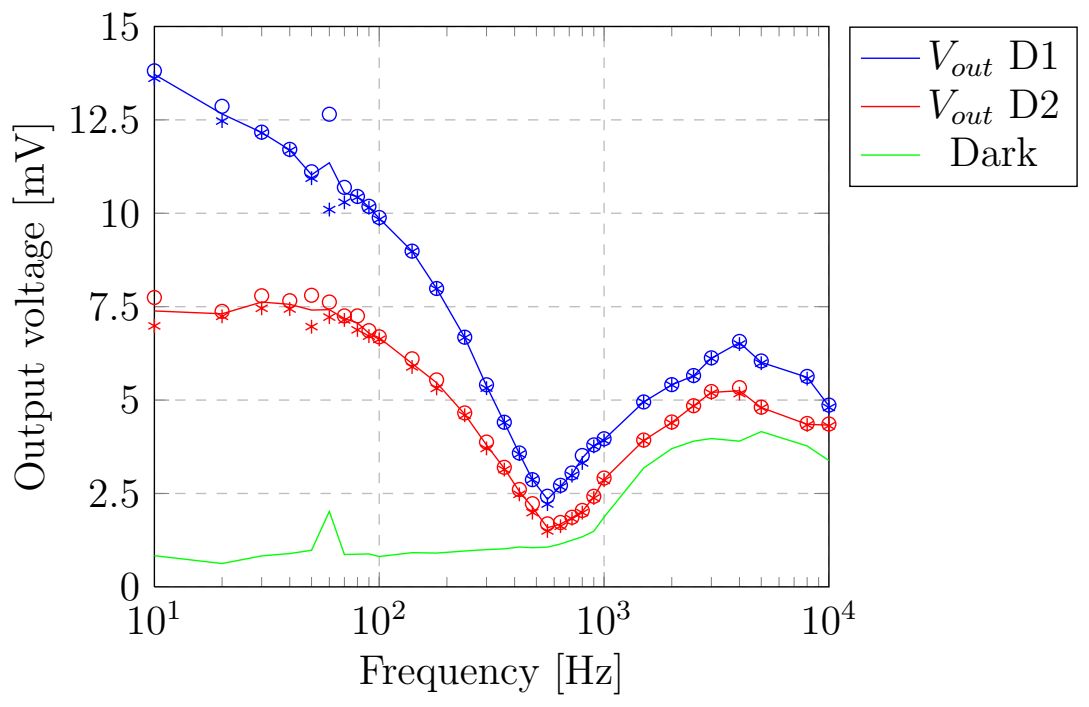

(b) Devices Size D

Figure 5.9: Frequency dependant response of bR/PEDOT:PSS device devices of size $\mathrm{C}$ and $\mathrm{D}$ 
The measurements seem to follow the expected trend: Turning on the light will increase the resistance of the PEDOT:PSS, which will in turn lower the voltage on the fixed resistor in the voltage divider as per Equation 5.8.

The response dependency on frequency also seems to follow the bR photocycle. To better understand this, it would be simpler to model the laser output as a square wave where every "on" cycle represents an irradiation of photons upon the bR.

For lower frequencies, the period of the square wave would be less than that of the $\mathrm{M}$ to bR state transition $(10 \mathrm{mS})$, meaning that in between irradiations there is plenty of time for the bR to reabsorb protons it would be pumping into the PEDOT:PSS, effectively decreasing the efficiency of the proton pump and causing minor de-doping and ergo small changes in resistance. As the frequency increases, the period of the laser starts to approach that of the proton re-uptake of bR, so more protons are being pumped into the PEDOT:PSS than are being pumped out, creating a larger differential in resistance and lowering the voltage of the fixed resistor. As the frequency increases, the light is now pumping much faster than the $M$ to bR step and starts to approach the period of bR to $\mathrm{M}$ state, meaning that light is being pumped faster than the bR can absorb it, leading to the saturation of the proton pump.

Since the output of the of the LIA is the total magnitude of the AC signal, an increase in voltage magnitude indicates an increase in resistance magnitude.

Out of all the samples, the larger devices A, B (Figure 5.9) and C (Figure 5.10 (a)) seems to exhibit the behaviour closest to predicted, with the major change in resistance happening around 400-700 Hz (1.4-2.5 mSec). Only the smallest device D (Figure 5.10 (b)) shows a different behaviour, where the largest magnitude of voltage is found around the low frequencies.

An explanation to this could stem from the cytoplasmic side only having a certain number of protons available to pump they are not be replenished quickly. When the laser is turned on initially the bR pumps the $\mathrm{H}+$ from the surface into the film polarizing the membrane and changing the resistance, but then saturates. So for the smallest device, there would be a lower amount of protons and it would saturate faster. The output voltage for this device is also quite lower than the other ones, which seems to corroborate that theory. As previously mentioned, orientation of the bR molecules in the smaller devices may also be an issue, as having poor orientation can cause the proton gradient to be unbalanced when irradiated by light, which would be even more noticeable on a smaller device which has overall less bR molecules. A 
potentially interesting study could be to find if the trans-membrane chemical gradient of bR can can create a capacitance which would increase the RC time constant of the system, delaying the charge carriers being moved into the PEDOT:PSS layer and lowering the frequency of operation. 


\section{Chapter 6}

\section{Conclusion}

\subsection{Summary of Knowledge Contribution}

In this thesis, an all organic optoelectrical device was fabricated and characterized. The results show that the interfacing the bR and PEDOT:PSS can be done with relative ease, as the two materials are quite compatible. Their processing is overall fairly simple and compatible, as they both require similar temperature and humidity conditions, and present a potential low cost analogue to today's photosensors, both in the aspect of materials and processing.

In terms of processing, this thesis showed that deposition and patterning of the materials can be done with no expensive equipment, as the processing is all done at fairly low (if not room) temperature, with low cost shadow and emulsion masks for patterning, and without any extra chemical etchants which could potentially save in manufacturing costs. This however does not bode well for integration into standard modern semi-conductor processing. Because of that, these types of devices would be better used for sensing or interfacing applications where they can be their own standalone structure and then interfaced with other computational devices which use more standard CMOS processes. Other alternate "wet" processes were proposed as well, but to a less successful degree.

In terms of a physical response, the effect of device size on photoresponse, both the DC and frequency response of the device, as well as both the absorption spectrum and photocycle physical response of the bR to different wavelengths and photon flux were studied. Irradiating the device with different wavelength representing different levels in the absorption spectrum yielded the expected spectral response: irradiation

closer to the peak absorption of $570 \mathrm{~nm}$ demonstrated a larger change in resistance 
than irradiation at wavelengths closer to $630 \mathrm{~nm}$, indicating a larger pumping of protons from the bR to de-dope the PEDOT:PSS. By varying the intensity of the laser and creating different photon fluxes which incident with the bR, the effect of size of the devices was studied. Other previous works did not account for device size nor variable irradiation intensity. This work shows the importance of studying different sized devices, as there is a direct correlation between percentage of area irradiated in the device and the total resistance change observed in the device. This knowledge can allow for tuning of device size to the desired response, as smaller devices can exhibit larger changes in resistance for the same input compared to larger devices. This is especially important when thinking of the specifications needed for this device to be used as a visual input to an organic neural network, where the changes in resistance in materials act as the main "learning" methods.

Finally, the frequency response of the device was studied, as well as how device size affects it. This is the main area where this work differentiates from others, as all other works cited previously focused on a single size of PEDOT:PSS/bR device and how it responds to a on/off cycle, as opposed to a frequency modulated optical signal. Again it was found that in smaller devices, the frequency response seemed to follow the theory more: The proton re-uptake of the bR is the limiting step to how fast the device can respond to a light change. This can give insight into the potential application of the device as a sensing element for visible light, and how well it can respond to a transient image, ie motion sensing.

\subsection{Improvements and Future Work}

Various improvements could be implemented for future devices and experiments.. From the processing side, the mixture of conducting polymer used was very old and it was known that these older versions have problems with adhesion. Steps such as ultrasonication and filtering were taken to prevent this, but it was not fully successful. Perhaps buying new material could yield more uniform layers with better adhesion, as Baytron has most likely improved their formulations over the years. Because of the little amount of bR available, only 1 working device to be tasted was successfully made. To truly test the repeatability of the results, more wafers with multiple devices should be processed and tested. Unfortunately because of high price of $b R$, this was not feasible. Also, as already mentioned earlier, when performing EFS to dry the bR 
dispersions, a more appropriate amount of exposure time to the electric field could have yielded better alignment, therefore increasing the photoresponse of the devices.

On the testing side, to truly test the spectral response of the device, a reasonably high power (mWs) tunable source such as a monochromator would have been needed. Because the monochromator divides down the power of the light, a powerful light source would have been needed to be able to incite enough photon flux incident on the device (larger than $1 \mathrm{~mW}$ ), however this equipment was not available. A lot of the data collection was performed manually, so more accurate results could have been derived from all measurements by having a computer with extraction software connected to the GPIB of the LIA.

Further work on the frequency operation, especially in the field of characterizing parasitic capacitances, could vastly improve our understanding of the switching characteristic of the bR, and help improve the specifications needed to trigger photoresponses large enough to make them useful for sensing applications.

Overall, it seems that bR/PEDOT:PSS devices are an exciting and interesting approach to organic sensing. While there are some major challenges with environmental stability, their ease of processing and interfacing, relative cheapness and large, measurable response gives these types of devices a potential application as visible lights sensors, and could very well advance the field of Organic Neural Networks and Organic Sensors. 


\section{List of References}

[1] N. Vsevolodov, Biomolecular Electronics. Boston, Massachusetts: Birkhauser Boston, 1998.

[2] J. Herzfeld J. C. Lansing, "Magnetic resonance studies of the bacteriorhodopsin pump cycle," Biomolecular Structures, vol. 31, 2002.

[3] M. I. Samoilovich A. F. Belyanin E. P. Grebennikov A. V. Guiyanov, "Bacteriorhodopin - the basis of molecular superfast nanoelectronics," Nanotechnology, vol. 13, 2002.

[4] Z. Chen R. Birge, "Protein-based artificial retinas," Trends in Biotechnology, vol. 11, 1993.

[5] T. Fischer M. Neebe T. Juchem N. Hampp, "Biomolecular optical data storage and data encryption," IEEE transactions on nanobioscience, vol. 2.

[6] P. Saeedi J. Moosaabadi M. Behmanesh A. Eidi J. Mehrabadi, "Bacteriorhodopsin and its mutants allude a breakthrough impending to artificial retina construction and strategies for curing blindness," Journal of Paramedical Sciences, vol. 2.

[7] C. Skotheim T. Elsenbaumer R. Reynolds J. Edsr, Handbook of Conducting Polymers, 2nd ed. New York, NY: Marcel Dekker, Inc., 1998.

[8] J. Olmsted G. Williams R. Burk, Chemistry, 2nd Canadian Edition. Toronto, ON: Wiley, 2010.

[9] M. Stevens, Polymer Chemistry: An Introduction - Third Edition. Oxford, GB: Oxford University Press, 1998.

[10] S. P. McGarry, "Elec3908 notes," 2015.

[11] S. P. McGarry N. G. Tarr, "Fabrication and modeling of screen-printed active electrolytic polymer devices," Semicond. Sci. Technol., vol. 23, 2008.

[12] A. Elschner S. Kirchmeyer W. Lovenich U. Merker K. Reuter, PEDOT: Principles and applications of an intrinsically conducting polymer. Boca Raton, FL: Taylor and Francis Gorup, 2011.

[13] R. Gangopadhyay B. Das M. R. Molla, "How does pedot combine with pss? insights fromstructural studies," RSC Advances, vol. 4, 2014. 
[14] S. Timpanaroa M. Kemerinkb F. J. Touwslagerc M. De Kokc S. Schradera, "How does pedot combine with pss? insights fromstructural studies," Chemical Physics Letters, vol. 4-6, 2004.

[15] C.S. Pathak J.P. Singh R. Singh, "Effect of dimethyl sulfoxide on the electrical properties of pedot:pss/n-si heterojunction diodes," Current Applied Physics, vol. 15, 2015.

[16] A. Nardes et al., "Microscopic understanding of the anisotropic conductivity of pedot:pss thin films," Advanced Materials, vol. 19, 2007.

[17] W. Wang G. K. Knopf A. Bassi, "Bioelectronic imaging array based on bacteriorhodopsin film," IEEE transactions on nanobioscience, vol. 7.

[18] M. Frydrycha P. Silfstenb S Parkkinenc J Parkkinend T Jaaskelainene, "Color sensitive retina based on bacteriorhodopsin," Biosystems, vol. 54.

[19] G. Sheppard, The Synaptic Organization of the Brain. New York, NY: Oxford University Press, 2004.

[20] R. R. Birge et al., "Biomolecular electronics: Protein-based associative processors and volumetric memories," J. Phys. Chem, vol. 103, 1999.

[21] N.B. Gillespie et al., "Color sensitive retina based on bacteriorhodopsin," J. Phys. Chem, vol. 106, 2002.

[22] F Vollmer J Topolancik, "Methods, materials and devices for light manipulation with oriented molecular assemblies in micronscale photonic circuit elements with high-q or slow light," 2008.

[23] A Horsager et al., "Vectors for delivery of light sensitive proteins and methods of use," 2012.

[24] M. Ates T. Karazehir A. S. Sarac, "Conducting polymers and their applications," Current Physical Chemistry, vol. 2, 2002.

[25] J. C. Hill S. Kichmeyer et al, "Pedt:pss a solution-processable alternative to ito in transparent conductor applications," H. C. Starck Inc., 2002.

[26] Y. Xiao et al., "Surface plasmon-enhanced electroluminescence in organic light-emitting diodes incorporating au nanoparticles," Applied Physics Letters, vol. 100, 2012.

[27] Y. Xia J. Ouyang, "Pedot:pss films with significantly enhanced conductivities induced by preferential solvation with cosolvents and their application in polymer photovoltaic cells," J. Mater. Chem., vol. 21, 2008.

[28] S. Ghosh O. Inganas, "Conducting polymer hydrogels as 3d electrodes: Applications for supercapacitors," Advanced Materials, vol. 11, 1990.

[29] N. Arun K. S. Narayan, "Conducting polymers as antennas for probing biophysical activities," J. Phys. Chem., vol. 112, 2008. 
[30] A. Manoj K. S. Narayan, "Opto-electrical processes in a conducting polymerbacteriorhodopsin system," Applied Physics Letters, vol. 83, 2003.

[31] S. Takamatsu et al., "Flexible, organic light-pen input device with integrated display," Sensors and Actuators, vol. 135, 2008.

[32] T. Suni K. Henttinen I. Suni J. Makinen, "Effects of plasma activation on hydrophilic bonding of si and sio2," Journal of Electrochemical Society, vol. 149, 2002.

[33] H. Tompkins E. A. Irene, Handbook of Ellipsometry. Norwich, NY: William Andrew Publishing, 2006.

[34] B. Charlot et al., "Micropatterning pedot-pss layers," Microsyst Technol, vol. 19, 2013

[35] A. Khaldi C. Plesse, "Conducting interpenetrating polymer network sized to fabricate microactuators," Applied Physics Letters, vol. 98, 2013.

[36] S. Ouyang et al., "Surface patterning of pedot:pss by photolithography for organic electronic devices," Journal of Nanomaterials, vol. 2015, 2014.

[37] U. Lang P. Rust J. Dual, "Towards fully polymeric mems: Fabrication and testing of pedot/pss strain gauges," Microelectronic Engineering, vol. 85, 2008.

[38] J. He et al., "Photoelectric properties of oriented bacteriorhodopsin/polycation multilayers by electrostatic layer-by-layer assembly," J. Phys. Chem., vol. 102, 1998.

[39] F. M. Smits, "Measurement of the resistivities with a four-point probe," 1957.

[40] D. C. Giancoli, Physics for Scientists and Engineers with Modern Physics (4th Edition). New Jersey, NY: Pearson, 2008.

[41] J. Min H. Choi J. Choi W. Lee, "Optimal fabrication condition of bacteriorhodopsin films by electrophoretic sedimentation technique," Supramolecular Science, vol. 5, 1998. 